\title{
Low-Frequency Ripple-Shaping Controller for Operation of Non-Inverting Buck-Boost Converters Near Step-Up STEP-DOWN BOUNDARY
}

by

\author{
Yuqing Zhang
}

A thesis submitted in conformity with the requirements for the degree of Master of Applied Science

The Edward S. Rogers Sr. Department of Electrical and Computer Engineering University of Toronto

(c) Copyright 2018 by Yuqing Zhang 


\begin{abstract}
Low-Frequency Ripple-Shaping Controller for Operation of Non-Inverting Buck-Boost Converters Near Step-Up Step-Down Boundary

Yuqing Zhang

Master of Applied Science

The Edward S. Rogers Sr. Department of Electrical and Computer Engineering University of Toronto

2018
\end{abstract}

The work presented in this thesis focuses on the elimination of step-up step-down mode transition problems that occur in low power dc-dc noninverting buck-boost (NIBB) converters, while also significantly improving power processing efficiency compared to existing state-of-the-art solutions. Near the step-down step-up boundary, the introduced controller enters a low-frequency ripple shaping mode of operation that allows lower switching frequency than in conventional buck or boost modes without paying a penalty of increased inductor current ripple, i.e. higher RMS current. As a result, both switching, and conduction losses are reduced. In addition, multi-level non-inverting buck-boost (ML-NIBB) is presented as a potential alternative to the conventional NIBB. Compared to the conventional architecture, the ML-NIBB converter has the potential to reduce the total volume and increase power processing efficiency. An extended version of the ripple-shaping controller is presented and used to preserve the smooth transition between step-down and step-up mode. The effectiveness of the presented ripple-shaping controllers is demonstrated with two $1.8 \mathrm{~V}$ to $5 \mathrm{~V}$ input, $3.3 \mathrm{~V} / 10 \mathrm{~W}$ experimental prototypes, for both NIBB and ML-NIBB topologies. 


\section{Acknowledgements}

First of all, I would like to thank my supervisor, Prof. Aleksandar Prodic, for giving me the opportunity of a life time by letting me be a part of your research group. Your passion and love for power electronics enlightens me every single day and have made my learning experience at University of Toronto truly an amazing journey. I am deeply grateful for the knowledge, brilliant ideas, and guidance you have generously provided to me not only on my project but also on life in general. Your encouragement, trust and endless help mean the world to me. I am so lucky!

Moreover, I would like to thank Ahsan for being an amazing mentor. Your thoughts and critical suggestions made a huge difference on my project!

I also want to use this opportunity to thank Giovanni Frattini, Maurizio Granato, and Giacomo Calabrese from Texas Instruments. I really appreciate you taking the time out of your busy work schedules to offer me your invaluable industry insights and guidance. Also, I want to give a big shout-out to the Texas Instruments Kilby lab. Without your generous financial support, nothing would have been possible!

To my lab mates and good friends, Andrija, Basil, Gianluca, Ivan, Jasmine, Mia, Michael, Nenad, Tim, Tom and Sam. Thank you guys for providing such a stimulating and fun environment for me to be around. I have learned so much. I am so lucky to be surrounded by you all throughout my study here. Thank you for all the help and unforgettably delightful time we have spent together. You guys are the best lab mates someone could ever ask for!

Furthermore, to my sweetest girlfriends, Rebecca, Sandy, Scarlet and Victoria. There is a saying that behind every strong electrical engineer is an army of supportive girls who got her back. Ok I just made that up:) but it is indeed true that I can never appreciate you enough for the love and joy you have given to me. You are the sweetest, most fun and loving group of girls who have always be here for me and constantly inspire me to be a better person. I love you all!

Last but never the least, I want to dedicate all my work to my dear parents, the people I love the most! I know that I have not being able to be around enough for all the family gatherings and holidays. But you never put a blame on me. Instead, you work hard so that I do not have to worry about anything but to focus on my study and research here. Thank you, my dearest parents, for all the unconditional love, support 
and understanding! I am reminded every day of my life, by all of the amazing things around me, that how lucky I am to have you two as my parents! I love you so much! 


\section{Contents}

$\begin{array}{ll}\text { Acknowledgements } & \text { iii }\end{array}$

Table of Contents $\quad$ v

List of Tables $\quad$ vii

List of Figures $\quad$ viii

List of Abbreviations $\quad$ xi

1 Introduction $\quad 1$

1.1 Introduction and Motivation . . . . . . . . . . . . . . 1

1.2 Thesis Objectives . . . . . . . . . . . . . . . . 6

1.3 Thesis Outline . . . . . . . . . . . . . . . 6

2 Prior Art $\quad 8$

2.1 NIBB Transition Issues . . . . . . . . . . . . . . . . . 8

2.2 Control based solutions . . . . . . . . . . . . . . . . . . . . . 10

2.2.1 Tri-interval operation . . . . . . . . . . . . . . . 11

2.2.2 Mixed-mode operation . . . . . . . . . . . . . 12

2.2.3 Hysteretic Current Mode Control . . . . . . . . . . . . . . 13

3 Low-frequency ripple-shaping mode $\quad 15$

3.1 Introduction . . . . . . . . . . . . . . . . . . . . 15

3.2 Principle of Operation . . . . . . . . . . . . . . . 16

3.2.1 Conversion Ratio of RS mode . . . . . . . . . . . . . . . . . 17

3.2.2 Inductor current level and ripple reduction . . . . . . . . . . . . . 18

3.3 Dual-Frequency Modulation . . . . . . . . . . . . . . . . . . . 21

3.4 Seamless Transition . . . . . . . . . . . . . . . . . . . . 22 
3.4 .1 Mode Selection . . . . . . . . . . . . . . . . . . 22

3.4 .2 Compensator Reset . . . . . . . . . . . . . . . . . . 23

3.4 .3 Frequency Scaling . . . . . . . . . . . . . . . 25

3.5 Practical Implementation . . . . . . . . . . . . . . . . . 26

3.5 .1 Average CPM . . . . . . . . . . . . . 26

3.5.2 Experimental Results . . . . . . . . . . . . . . . . . 28

3.6 Conclusion . . . . . . . . . . . . . . . . . . . . . . . . . . 34

4 Multilevel NIBB $\quad 36$

4.1 Introduction . . . . . . . . . . . . . . . . 36

4.2 Principle of Operation . . . . . . . . . . . . . . . . . 38

4.2 .1 ML-RS Mode . . . . . . . . . . . . . . . . . . . . 40

4.3 Inductor volume reduction and efficiency improvements . . . . . . . . . 43

4.3.1 Inductor volume reduction . . . . . . . . . . . . . . . . . 43

4.3 .2 Efficiency improvement . . . . . . . . . . . . . . . . . 47

4.4 Practical Implementation . . . . . . . . . . . . . . . . . . . . . . 48

4.4 .1 Discrete prototypes parameters . . . . . . . . . . . . 49

4.4 .2 Efficiency Comparison . . . . . . . . . . . . . . 51

4.4 .3 ML-NIBB Operation . . . . . . . . . . . . . . 54

4.4 .4 Flying capacitor control . . . . . . . . . . . . 58

4.5 Conclusion . . . . . . . . . . . . . . . . . . . . . . . 62

5 Conclusion $\quad 63$

5.0 .1 Future work . . . . . . . . . . . . . . . . . 64 


\section{List of Tables}

3.1 Converter Parameters . . . . . . . . . . . . . . . . . . . 28

4.1 Converter parameters for RS controller verification . . . . . . . . . . 49

4.2 Converter Parameters for efficiency comparison . . . . . . . . . . . . 50 


\section{List of Figures}

1.1 Power stage of conventional non-inverting buck-boost converter . . . . . 2

1.2 iPhone X PCB area teardown. https://www.ifixit.com/Teardown/iPhone+X+ Teardown $/ 98975 \ldots \ldots \ldots$. . . . . . . . . . . . . 4

1.3 PCB area breakdown of iPhone X mother board . . . . . . . . . . . . . . 4

1.4 Multi-level Non-inverting buck-boost converter . . . . . . . . . . . . 5

2.1 Non-inverting buck boost converter . . . . . . . . . . . . . . . . 8

2.1 Non-inverting buck boost converter operates in buck mode or boost mode 9

2.2 Transition goal:preseve the continiuty characteristic . . . . . . . . . . . 9

2.3 Increased output voltage due to pulse skipping . . . . . . . . . . . . 10

2.4 Inductor current in buck mode, buffer mode and boost mode(from left to right) . . . . . . . . . . . . . . . . . . . 12

2.5 Switching scheme at the boundary of step-down and step-up . . . . . . 13

2.6 Inductor current instability during the transition . . . . . . . . . . . . 13

2.7 Hysteretic control mode transition between buck and boost mode . . . . 14

3.1 Non-inverting buck-boost converter . . . . . . . . . . . . . . 16

3.2 Inductor current waveform comparison between buck-boost mode and RS mode . . . . . . . . . . . . . . . . . . 16

3.3 Key waveforms of ripple-shaping mode including gating signals, switching node voltage, inductor current(left) and operation subintervals(right) . . 17

3.4 Inductor current waveforms in step-down mode and step-up mode (a) shows the step-down case where slope of initial phase and $t_{2}$ is greater than $t_{1}$. (b) shows the step-up case where slope of initial phase is negative and $t_{2}$ is smaller than $t_{1} \ldots \ldots \ldots \ldots$. . . . . . . . . . . 18

3.5 Inductor current in buck boost mode, tri-interval mode and Ripple-shaping mode ............................ 19

3.6 Normalized RMS current comparison in the dead zone . . . . . . . . . . 21 
3.7 Inductor current waveform in RS mode under different frequency . . . . . 22

3.8 Hysteretic Band . . . . . . . . . . . . . . . . . . . . . . . . . . . . 23

3.9 Duty ratio change with compensator reset(a) and without compensator $\operatorname{reset}(\mathrm{b}) \ldots \ldots \ldots \ldots \ldots 24 \ldots \ldots \ldots$

3.10 Experimental results verify the effectiveness of duty ratio reset technique 25

3.11 Inductor current waveform change from boost mode to RS mode . . . . . 26

3.12 Inductor current waveform change from buck mode to RS mode . . . . . 26

3.13 Non-inverting buck-boost converter regulated with a low-frequency rippleshaping controller, implemented through digital average current mode con-

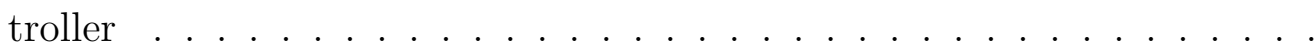

3.14 Steady state operation in (a) buck (b) boost (c,d) RS step-down (e,f) RS step-up mode; For all the waveforms: Ch.1 (Orange): $2 \mathrm{~V} /$ div output switching node voltage; Ch.2 (Green): $2 \mathrm{~V} /$ div input switching node voltage; Ch.3 (Purple): $500 \mathrm{mV} /$ div output voltage; Ch.4 (Pink): $500 \mathrm{mV} /$ div inductor current;

3.15 Mode transition between (a,b,c,d) buck and RS SD (e,f,g,h) boost and RS SU $(\mathrm{i}, \mathrm{j}, \mathrm{k}, \mathrm{l})$ RS SD and RS SU; For all the waveforms: Ch.1 (Orange): $2 \mathrm{~V} /$ div output switching node voltage; Ch.2 (Green): $2 \mathrm{~V} /$ div input switching node voltage; Ch.3 (Purple): $500 \mathrm{mV} /$ div output voltage; Ch.4 (Pink): $500 \mathrm{mV} /$ div inductor current; . . . . . . . . . . . . 32

3.16 Power losses comparison . . . . . . . . . . . . . . . . . . 33

3.17 Efficiency comparison between RS and other state of art . . . . . . . . . 34

4.1 Multi-level Non-inverting buck-boost converter . . . . . . . . . . . . . . . 37

4.2 Subintervals of RS operation . . . . . . . . . . . . . . . . . . 39

4.3 3-level buck operation when duty ratio is smaller than 0.5 (left) and when duty ratio is larger than 0.5 (right) . . . . . . . . . . . . . . . 40

4.4 3-level boost operation when duty ratio is smaller than 0.5 (left) and when duty ratio is larger than 0.5 (right) . . . . . . . . . . . . . . . . . 40

4.5 Key waveforms of multi-level ripple-shaping including switching sequence, switching node voltages, corresponding inductor current waveform . . . . 42

4.6 Normalized inductor values in NIBB and ML-NIBB with respect to worst inductance in transition region . . . . . . . . . . . . . . . . . 47

4.7 Multi-level Non-inverting buck-boost converter regulated with low-frequency multi-level ripple-shaping controller,implemented through digital average current mode controller . . . . . . . . . . . . . . . . . . . . . 
4.8 Efficiency comparison between ML-RS and conventional RS . . . . . . .

4.9 Efficiency comparison between 3-level buck and 2-level buck in duty ratio

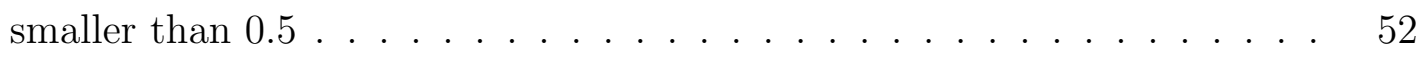

4.10 Efficiency comparison between 3-level buck and 2-level buck in duty ratio

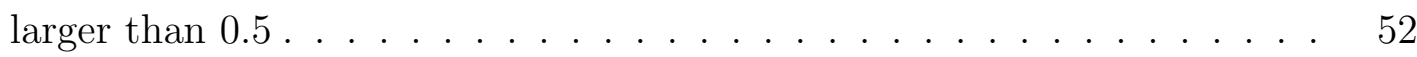

4.11 Efficiency comparison between 3-level boost and 2-level boost in duty ratio

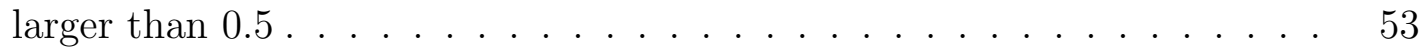

4.12 Efficiency comparison between 3-level boost and 2-level boost in duty ratio smaller than $0.5 \ldots \ldots \ldots \ldots \ldots \ldots \ldots \ldots$

4.13 Steady state operation in (a) buck (b) boost (c,d) RS step-down (e,f) RS step-up mode; For all the waveforms: Ch.1 (Orange): $2 \mathrm{~V} /$ div output switching node voltage; Ch.2 (Green): $2 \mathrm{~V} /$ div input switching node voltage; Ch.3 (Purple): $500 \mathrm{mV} /$ div output voltage; Ch.4 (Pink): $500 \mathrm{mV} /$ div inductor current; . . . . . . . . . . . . . . .

4.14 Mode transition between (a,b,c,d) buck and RS SD (e,f,g,h) boost and RS SU (i,j) RS SD and RS SU; For all the waveforms: Ch.1 (Orange): 2V/div output switching node voltage; Ch.2 (Green): 2V/div input switching node voltage; Ch.3 (Purple): 500mV/div output voltage; Ch.4 (Pink): $500 \mathrm{mV} /$ div inductor current $\ldots \ldots \ldots \ldots \ldots . \ldots . \ldots 58$

4.15 Key waveforms in ML-RS step down mode with flying capacitor voltage

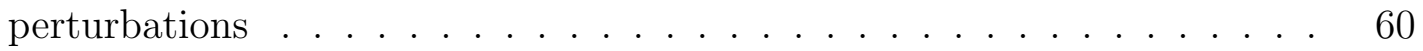

4.16 Duty ratio offsetting mechanism balance the flying capacitor voltages . . 60

4.17 Duty ratio offsetting mechanism in RS mode . . . . . . . . . . . . . . 61 


\section{List of Abbreviations}

$\begin{array}{ll}\text { SMPS } & \text { Switch Mode Power Supply } \\ \text { PID } & \text { Proportional Integral Derivative } \\ \text { RS } & \text { Ripple Shaping } \\ \text { IC } & \text { Integrated Circuit } \\ \text { NIBB } & \text { Non-Inverting Buck-Boost } \\ \text { RMS } & \text { Root Mean Square } \\ \text { DPWM } & \text { Digital Pulse Width Modulator } \\ \text { 3L } & \text { 3-Level } \\ \text { CCM } & \text { Continuous Conduction Mode } \\ \text { DCM } & \text { Discontinuous Conduction Mode } \\ \text { ML } & \text { Multilevel } \\ \text { ML-RS } & \text { Multilevel Ripple-Shaping } \\ \text { ML-NIBB } & \text { Multilevel Non-Inverting Buck-Boost } \\ \text { FOM } & \text { Figure of merit } \\ \text { CPM } & \text { Current Programmed Mode Control } \\ \text { PCB } & \text { Printed Circuit Board }\end{array}$




\section{Chapter 1}

\section{Introduction}

\subsection{Introduction and Motivation}

Power management is an essential part of all electronic systems and regulated power supplies are required for the proper operation of the majority of both analog and digital electronics. A power supply can be roughly divided into two parts: converter and controller circuit. The converter is a circuit which transfers power from sources to loads (e.g. CPUs, microprocessors, RF amplifiers, and LED screen backlights, etc.). The control circuit monitors the state variables of the system and adjusts converter parameters to regulate the output voltage or current to a specified value. The type of dc power management subsystem to be used in a given application depends on the input and output voltage ranges. For battery-powered portable applications, like smart phones, portable audio players, laptops, etc., long battery operation time is required $[1,2,3,4]$. In order to effectively use all available battery capacity, a front end converter that is able to both step-up and step-down the input voltage is often needed to provide a well regulated output bus voltage. This is because the battery voltage, which varies with temperature, state of charge, etc, can go above or below the desired output voltage.

Many converters have step up/step down functionality, including the buck-boost, Cuk, Zeta, and SEPIC converter, but they are not without their drawbacks for the low power applications targeted in this thesis. The converters listed above all have an output voltage that is negative with respect to the input voltage i.e. reverse the input voltage, and/or they utilize more than one inductor. For portable battery powered applications these two aspects are typically undesirable due to the increased area associated with multiple inductors and EMI issues that can arise from the lack of common ground between the input and the battery. The non-inverting buck-boost (NIBB) converter shown in Fig 
1.1 is particularly well suited for the applications, as it has non-inverting output and uses only a single inductor, which is relatively small compared to other solutions [5]. As such, the NIBB is almost exclusively used in size and cost constrained portable power applications.

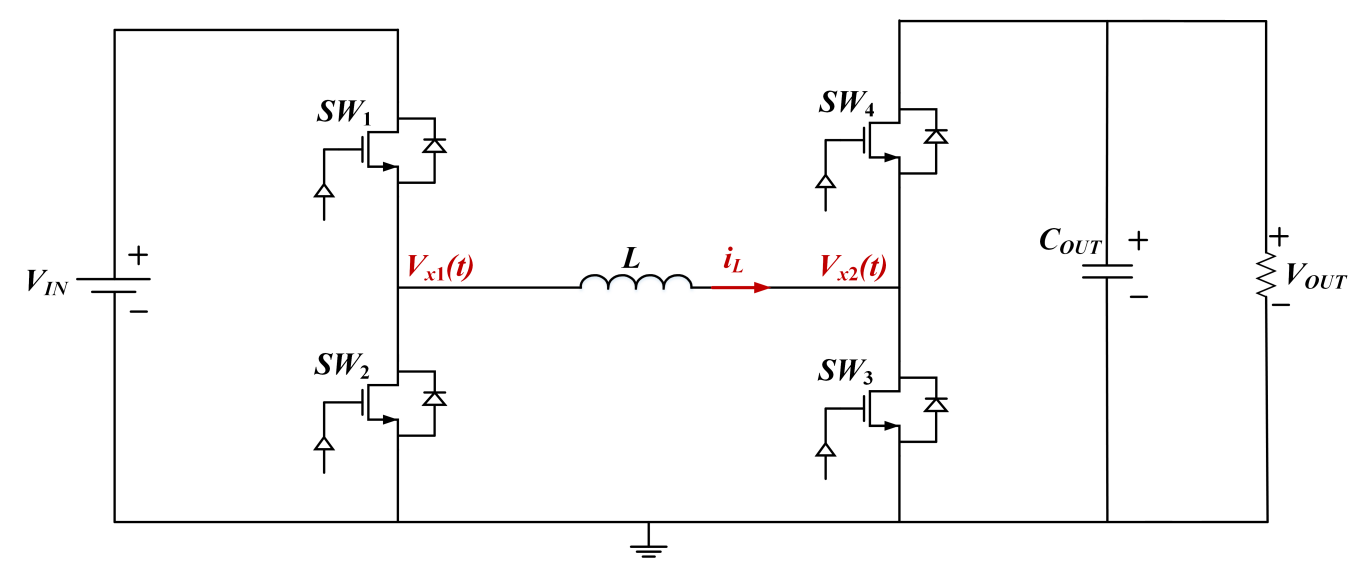

Figure 1.1: Power stage of conventional non-inverting buck-boost converter

Commonly, the NIBB operates in 3 distinct modes depending on the battery voltage. When the battery voltage is significantly higher than the output voltage, the NIBB operates in buck mode. When the battery voltage is significantly lower than the output voltage, the NIBB operates in boost mode. The NIBB only operates in a buck-boost mode when the battery and the output voltage are similar. This is done because even though the NIBB could operate in buck-boost mode over the whole range, the converter typically has lower power processing efficiency and a higher current rating for the inductor compared to the other two modes. Therefore, buck-boost mode needs to be avoided to achieve higher power processing efficiency during the transtion. However, there is a problem related to the practical implementation of the converter. Ideally, a buck converter can adjust its output voltage from zero all the way up to the input voltage, but practically this impossible due to the non-zero transition time when turning on and off power semiconductor devices. As a result, duty ratios of converters are often limited to between $5 \%$ and $95 \%$. This creates a discontinuity between the operation modes of the NIBB and sets the question: how does one transition between buck and boost while improving power processing efficiency?

A significant amount of research has been done regarding the operation when input and output voltages are similar in NIBB converter. Specific implementations have been investigated $[2,3,4,6,7]$, the discontinuity between buck and boost modes, and specific 
solutions to the transition have been investigated. Finding a control scheme for the smooth transition between step-up and step-down operation while preserving high power processing efficiency has been a challenge [8, 9]. Multiple control methods $[10,11,12,13$, $14,15]$ proposed in recent years aim at avoiding the buck-boost mode while reducing the average inductor current and switching losses at the same time. These control methods will be reviewed in Chapter 2 .

Most control schemes suggested in previous art utilize analog control. While implementation of analog control is well understood, it is not without its drawbacks. Specifically, increasing the number of switching elements while requiring explicit control of each element proves challenging for an entirely analog control system. Digital control has seen wider adoption for low-power converters in recent years [16]. It opened up the possibility of more complex control schemes for more effective results.

Another challenge that currently attracts research efforts in power management is the requirement for higher power density, i.e. more power processing capability within smaller system volume. Increasing functionality of mobile devices and low-power systems has resulted in tighter constraints on the area and cost dedicated to the on board power management systems. In order to address this problem, volume reduction of passive components, i.e. inductors and capacitors, is the key to achieve miniaturization of the power management system. For example, the size of inductors of NIBB, which dominates the overall converter size, is a large obstacle in further minimization and increase of capabilities of emerging portable devices. The inductors occupy a relatively large volume that could potentially also be used by some emerging functional blocks.

Fig 1.2 is the bottom view of the mother board of the iPhone $\mathrm{X}$. The passive components are shaded in light blue. As shown in the Fig 1.3, passive components consume around $23 \%$ of the overall PCB area and inductors occupy the majority of the area shaded in blue. Two power management ICs circled in orange and pink take up around $9 \%$ of the overall area. Decreasing the passive component volume can open up the possibility of adding more functional blocks without increasing system volume or simply reducing the overall system volume. 


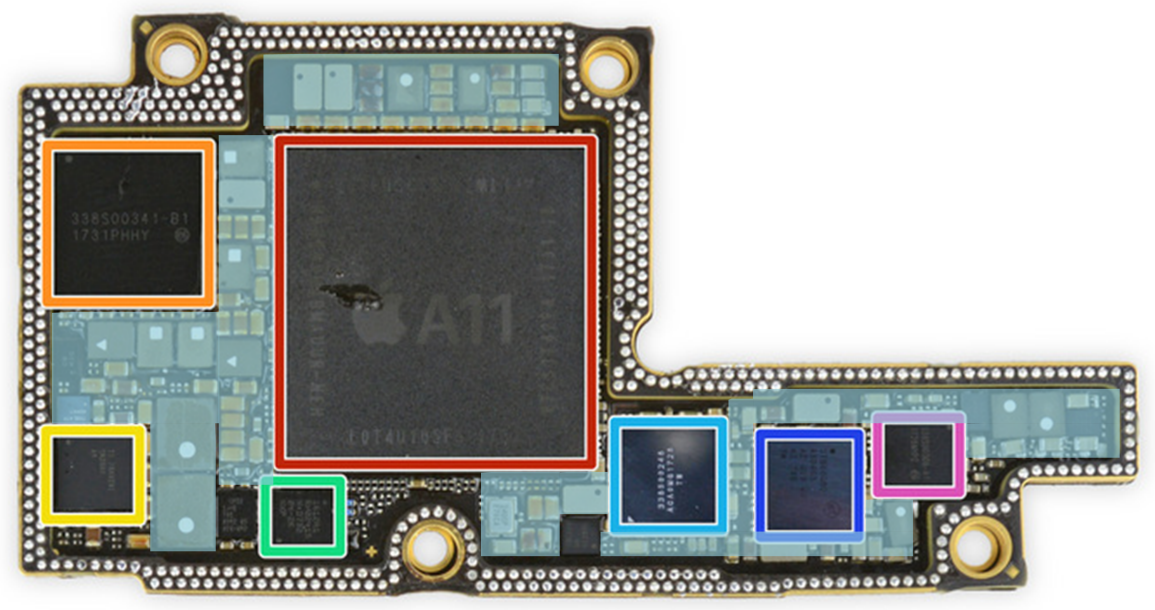

Figure 1.2: iPhone X PCB area teardown. https://www.ifixit.com/Teardown/iPhone+X+ Teardown/98975

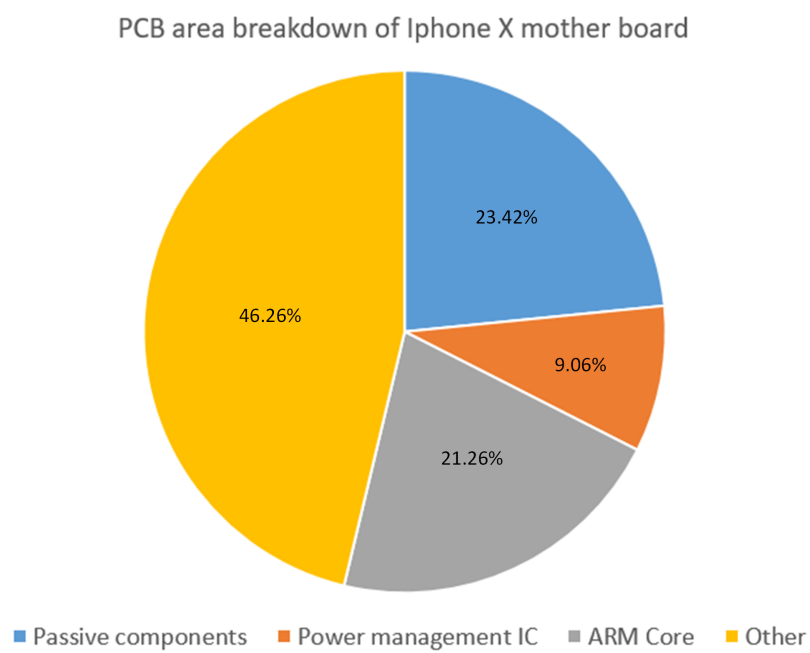

Figure 1.3: PCB area breakdown of iPhone $\mathrm{X}$ mother board 
To a first order approximation, the volume of passive components is related to the energy storage of the element. For inductor, $W_{L}=1 / 2 \cdot L \cdot I_{\text {peak }}{ }^{2}$ and for capacitor $W_{C}=1 / 2 \cdot C \cdot V^{2}$. Furthermore, inductance $L$ is proportional to the voltage swing across the inductor and inversely proportional to frequency of the desired inductor current ripple in power converters [17]. Therefore, increasing switching frequency can be a solution for reducing the inductor volume. However, it is often not optimal due to the increased switching losses. Another solution is to decrease the voltage swing across the inductor, which relies on emerging topologies and control schemes.

The flying capacitor (FC) multi-level buck converter has been utilized in high-power applications due to the reduction of both voltage stress across the power switches and voltage swing of the inductor $[18,19]$. These advantages come from the utilization of flying capacitor, switching node voltage is $1 /(N-1)$ of the input voltage, where $N$ is the number of switching node voltage level. This topology allows for a significant reduction of the inductor size and semiconductor power losses. In recent years, interest of using multi-level converter in low-power application has been increased [20]. A derivation from 3-level buck, which includes 3-level buck in series of 3-level boost with an inductor in between could be an attractive alternative option to conventional NIBB Fig.4.1. The advantages of multi-level topology could be adapted in this converter. The comparison between conventional NIBB and this architecture in terms of volume and power processing efficiency will be analyzed in chapter 4 .

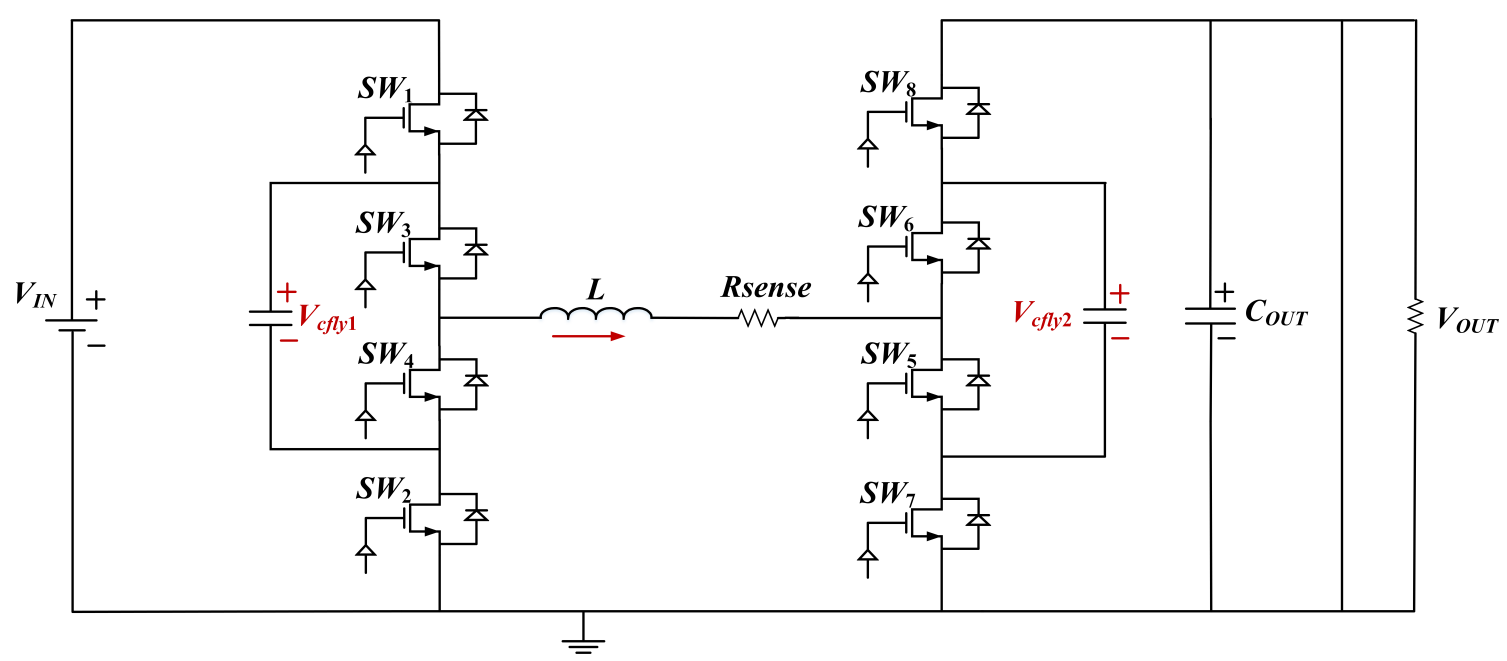

Figure 1.4: Multi-level Non-inverting buck-boost converter 


\subsection{Thesis Objectives}

The objective of this thesis is to introduce the practical control-based solution for the smooth transition between step-up and step-down modes of the conventional NIBB. The transition problems and related control challenges occurring at the boundary of step-down and step-up region are addressed. A novel, low-frequency ripple-shaping (RS) mode will be introduced and discussed. The RS controller not only solves the transition issues but also improves power processing efficiency of both NIBB and ML-NIBB converters at the boundary operation region, as compared to some recently developed methods used in the products on the market. The RS mode operation can maintain low current ripple while drastically reducing switching losses even in the presence of heavy loads. It will be shown that this mode also reduces conduction losses, which provides significant power processing efficiency improvements. The practical solutions for implementing the introduced RS method are presented. Furthermore, ML-NIBB converter is introduced as an alternative option to conventional NIBB. The comparison analysis between MLNIBB and conventional NIBB is shown to prove the volume reduction and efficiency improvements in ML-NIBB. The effectiveness of the control method has been verified with both conventional NIBB and ML-NIBB converter prototypes, using a FPGA based digital controller.

\subsection{Thesis Outline}

Chapter 2 reviews prior state-of-the-art. Several control methods that provide a transition between step-down and step-up operation are reviewed.

Chapter 3 introduces ripple-shaping (RS) mode. The features of this mode are discussed in detail. Practical implementation challenges are addressed, and corresponding solutions are presented. Efficiency comparison to state-of-the-art solutions are shown. The experimental results confirm that, inductor current level and ripple are reduced drastically and seamless transitions between each mode are achieved, while power processing efficiency when $V_{I N}$ is close to $V_{O U T}$ improves up to $6 \%$.

Chapter 4 presents comparison analysis between conventional NIBB and ML-NIBB in terms of the volume and power processing efficiency for the full operation regions. Results show that in ML-NIBB, higher power processing efficiency having power MOSFETs with better figure of merit (FOM) could be utlized. Furthermore, RS mode is extended to ML-RS to adapt the operation of ML-NIBB. Efficiency results comparison between NIBB 
and ML-NIBB in step down, step up and RS mode are presented and discussed. Steady state operation with flying capacitors control of ML-NIBB in all three regions are shown. Seamless transition between different modes are explored.

Chapter 5 presents conclusions and discuss future work. 


\section{Chapter 2}

\section{Prior Art}

To address the aforementioned transition problem in non-inverting buck-boost converter, a number of control based solutions have been proposed [10, 11, 12, 13, 14, 15, 21, 22, 23]. In this chapter, prior state of the art are reviewed.

\subsection{NIBB Transition Issues}

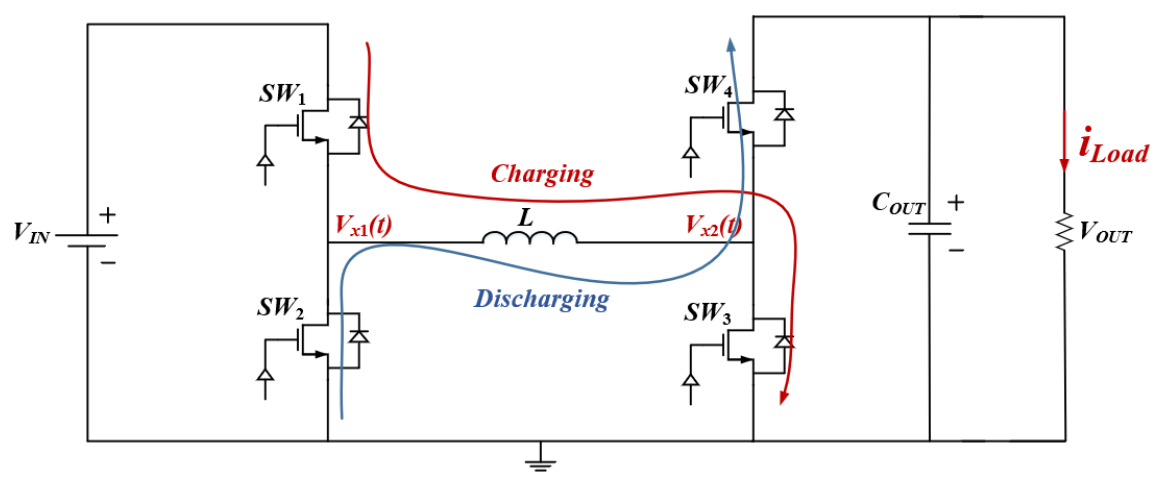

Figure 2.1: Non-inverting buck boost converter

NIBB converter shown in Fig 2.1 can operate in the buck-boost mode directly by switching between inductor charing and discharging phases. However, this mode is not desirable, mostly due to the low power processing efficiency and requirements for a larger inductor current, compared to the buck and boost operation. Therefore, NIBB converters usually operate in buck mode, when $V_{I N}$ is larger than $V_{O U T}$, where $S W_{4}$ is always on and $S W_{3}$ is always off. When $V_{I N}$ is smaller than $V_{O U T}$, the converter operates in boost mode, where $S W_{1}$ is always on and $S W_{2}$ is always off. When $V_{I N}$ is close to $V_{O U T}$, due to the discontinuity in voltage regulation at the boundary of buck and boost mode, smooth 
transition has been proven a challenge [8]. When it comes close to the mode transition, as buck duty ratio approaches to 1 or boost duty ratio approaches to 0 , a limitation arises as how close the duty ratio can actually approach to these two values (0 and 1$)$.

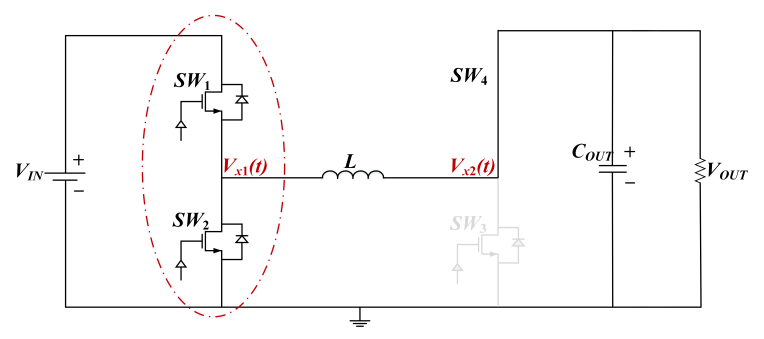

(a) Buck operation

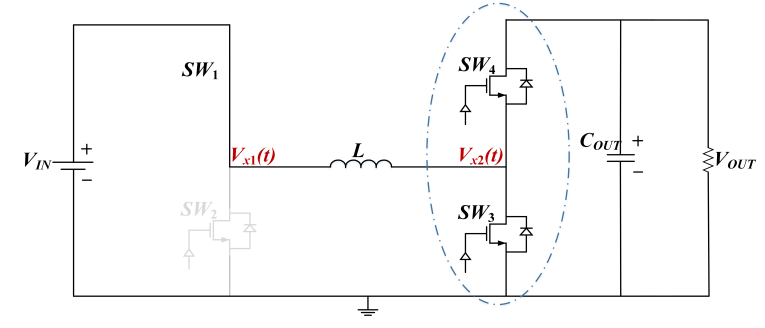

(b) Boost operation

Figure 2.1: Non-inverting buck boost converter operates in buck mode or boost mode

In practical implemenation, minimum rise/fall time of the driver for power switches and deadtime that is needed for switch commutation will limit the maximum or minimum achievable duty cycle [9]. Therefore, around the mode transition, the discontinuity happens in the conversion ratio vs. control signal characteristics shown in Fig 2.2a. This implies that no adequate control signal could be found to regulate the output voltage to be equal to reference voltage. Instead, limit cycle oscillation will happen, so that average output voltage tracks the reference but control signal bounces between 1 and maximum limitation. This results in inductor current instability and increased output voltage ripple as shown in Fig 2.3. Therefore, the goal for the smooth transition is preserving the continuity of the voltage gain vs. control signal characteristic as shown in Fig $2.2 \mathrm{~b}$ while minimizing converter power losses.

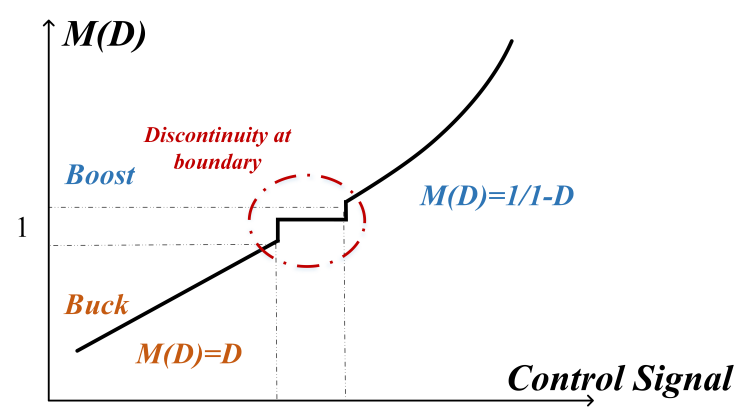

(a) Discontiuity at boundary

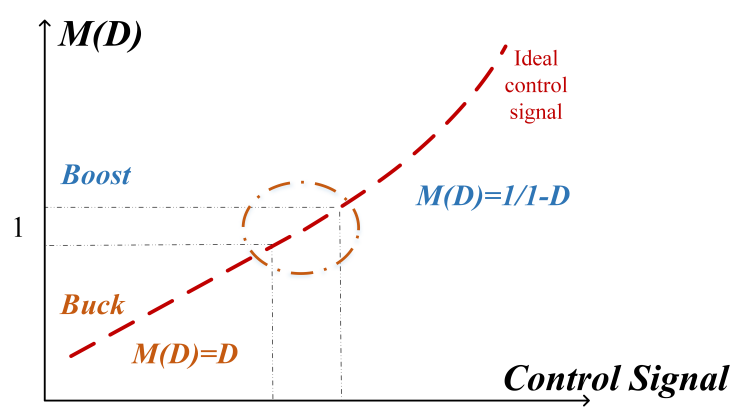

(b) Ideal transition

Figure 2.2: Transition goal:preseve the continiuty characteristic 


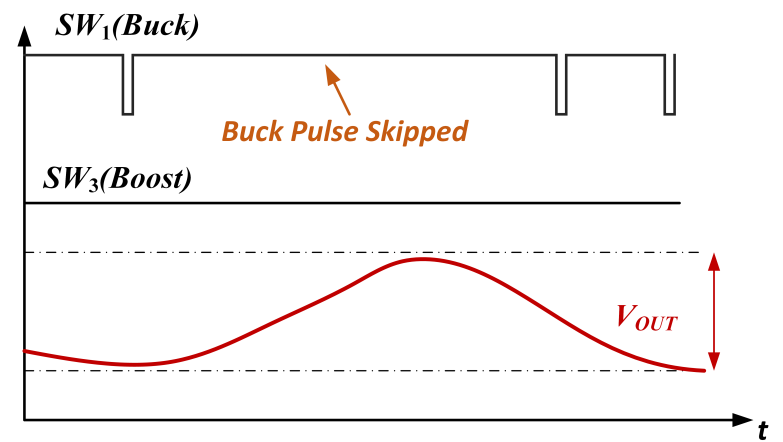

Figure 2.3: Increased output voltage due to pulse skipping

\subsection{Control based solutions}

The existing literature on smooth transition of NIBB can be classified into serval categories. In $[12,13,14,24]$, a third mode is introduced to eliminate the dead zone. The simplest approach is to operate converter in buck-boost mode with 0.5 duty ratio, with the price of doubling the switching losses and increasing conduction loss as well as inductor volume.

In order to decrease the conduction losses, tri-interval operation is introduced in [13]. $D_{\text {buck }}$ and $D_{\text {boost }}$ are individually specified to determine the conversion ratio. This kind of operation reduces the interval that energy only transfers to inductor and introduces an interval where input and output voltage are directly connected. However, switching losses are still doubled due to the 4 -switch operation. Solutions presented in $[12,14]$ proposed to halve the switching frequency in tri-interval operation. This mode of operation will be explained in more detail in subsection 2.2.1., where inside a buffer region converter operates in tri-interval manner with double switching period, to reduce both conduction and switching losses. Extra transition techniques are required to ensure smooth transition between the buck/boost mode to the halved switching frequency.

Another category of control methods is toggling between buck mode and boost mode $[15,23]$. Only two switches are active in one switching cycle which has no extra switching losses. In addition, inductor current is the average of buck cycle and boost cycle which is much smaller than that in the buck boost mode. A drawback of this method is instability of inductor current and output voltage during the transition. Instead of the buck mode and boost mode operating sequentially, when the input voltage is very close to the output voltage and more of a step down/up is needed, multiple buck/boost operation 
with single boost/buck mode will occur, which increase the inductor current and output voltage ripple. An example of this control method will be reviewed in detail in subsection 2.2 .2 .

In [11] the inductor current ripple amplitude is kept constant while the switching frequency is changed according to the value of $V_{I N}$ and $V_{O U T}$. This drastically reduces switching loss. In [11], hysteretic control is used on the inductor current for the whole range of operation. Mode detection and transition are automatically decided by the slope of the inductor current. This control scheme will be explained in further detail in subsection 2.2.3. When $V_{I N}$ is equal to $V_{O U T}$, the slope of the inductor current is 0 , which leads to no active mode switching.

\subsubsection{Tri-interval operation}

The control method proposed in $[12,14]$ is to provide a buffer stage between buck and boost to replace buck boost operation. In the dead zone, where required conversion ratio cannot be reached with just buck or boost mode, due to the limitation of power switches, this control technique combines both buck and boost operation. The switching sequence and corresponding inductor current is shown in Fig 2.4. Limit cycle oscillation is effectively eliminated by the insertion of boost operation in step down mode and buck operation in step up mode. Converter operates in such manner that there are three subintervals in each switching cycle. Even though four switches are operating to constitute one regulation cycle, there are only two switches turning on or off in one switching cycle. Therefore, minimum impact on the switching loss can be achieved. On top of that, the average inductor current depends on the duration of indirect energy transfer. Due the minimization of that interval, the average inductor current and ripple are reduced. This control method introduces buffer stage that combines buck and boost switching cycle to avoid duty ratio reaches its maximum or minimum limit and reduce inductor current level so as conduction losses at the same time. Converter must transition in and out of a half switching frequency dead zone mode on the basis of measurements of input and/or output voltages. This increases the complexity of mode transition to achieve minimum deviation of inductor current and output voltage. 


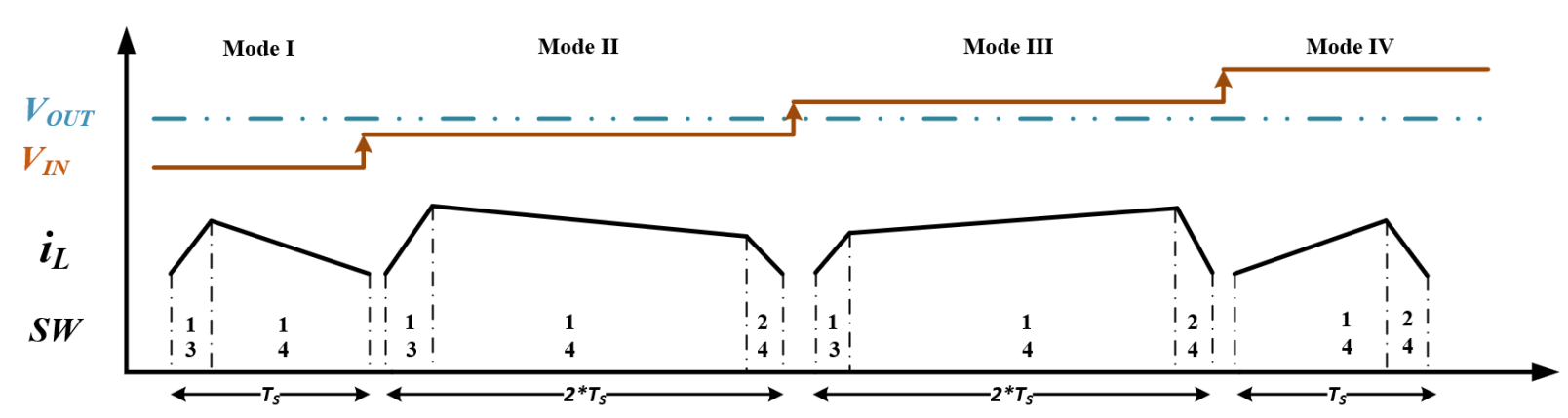

Figure 2.4: Inductor current in buck mode, buffer mode and boost mode(from left to right)

\subsubsection{Mixed-mode operation}

In [10], operations of NIBB are defined as initial phase, buck phase and boost phase shown in the Fig 3.3 respectively. As shown in the Fig 2.5, the duty ratio of the switches are determined by the compensator control signal $V_{C}$, buck and boost carriers and reset clock signal. The reset clock signal goes high every switching cycle to reset the operation into initial phase. And when the control signal intersects with the buck carrier, where duty ratio of boost switch is 0 , converter operates in buck phase with initial phase. While the control signal intersects with the boost carrier, duty ratio of buck switch is 1 , and converter switches between boost phase and initial phase. When $V_{I N}$ is close to $V_{O U T}$, the control signal could intersect with buck and boost carriers sequentially so that buck-boost mode is avoided. As result, converter operates in buck and boost mode interchangeably in the transition region. The advantages of this control method is no input voltage sensing is needed, auto mode selection is achieved by control signal $V_{C}$. From the efficiency perspective, average inductor current reduces to the average of buck and boost average current values. Also only two switches are switching in one switching cycle. Therefore, both conduction and switching losses are reduced. However, the pattern of buck or boost switching modes is not necessarily stable. In the range of transition, when more step down is needed, buck mode could be on for few cycles with one boost mode. Likewise, during the step-up transition, more boost mode will be operated than buck mode. This causes the instability of inductor current and output voltage. Fig 2.6 shows the inductor current instability during the transition using mixed-mode operation. The result was taken from the evaluation board of a commercial NIBB IC using this transition method [25]. 

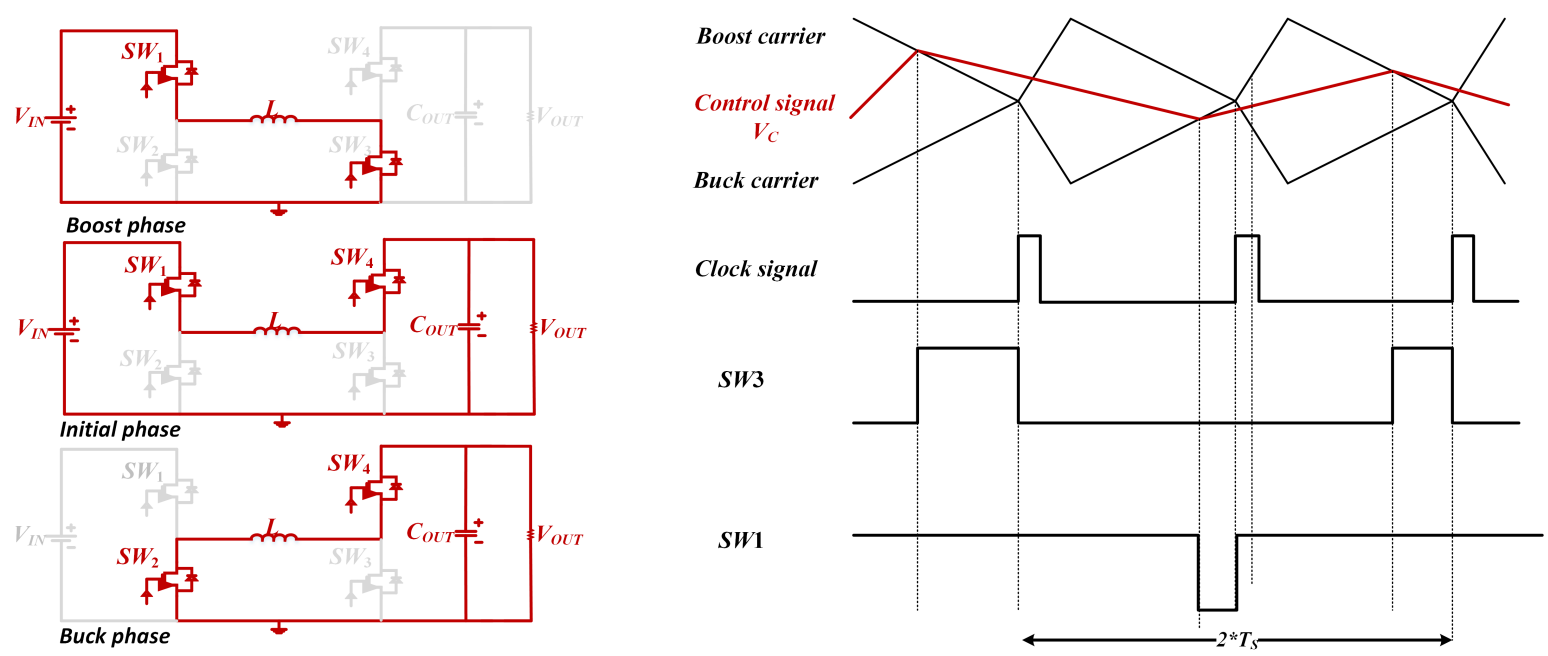

Figure 2.5: Switching scheme at the boundary of step-down and step-up

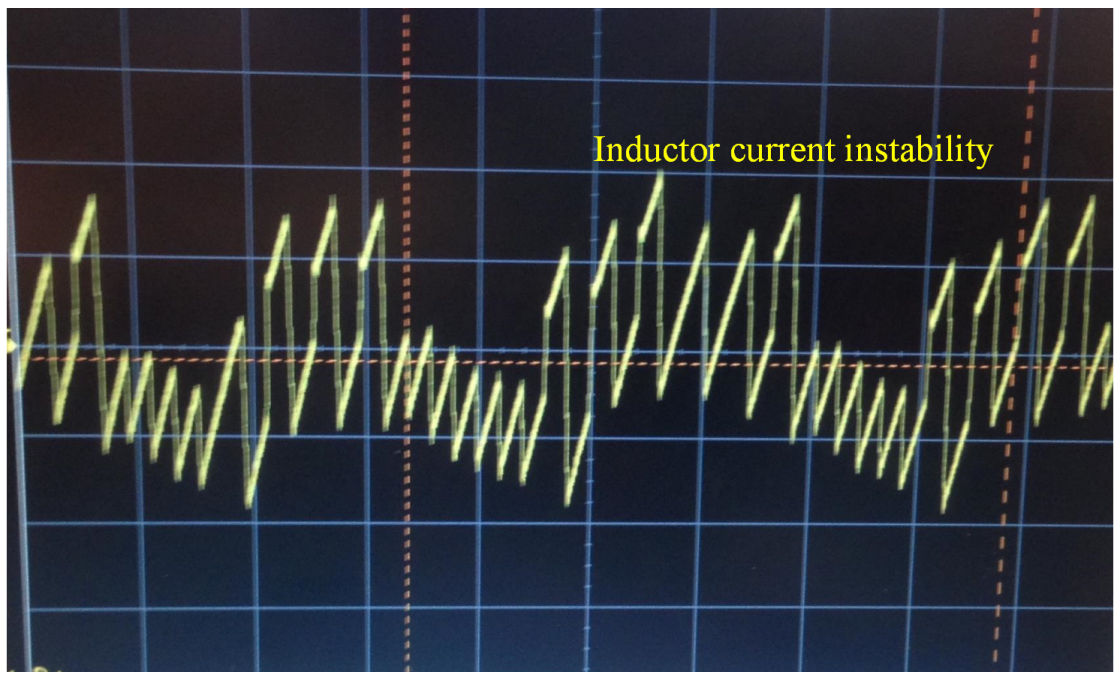

Figure 2.6: Inductor current instability during the transition

\subsubsection{Hysteretic Current Mode Control}

In [11], a hysteretic current mode controller is built for the smooth transition of NIBB while minimizing the power losses. The hysteretic window circuit generates two hysteretic windows, according to the control signal from compensator. Shown in Fig 2.7, $V_{I B}$ is the lower band and the $V_{I T}$ is the higher band of the window. Sensed inductor current is converted to a voltage signal and compared with the hysteretic band, to determine the switching pattern. The operational mode is decided by the inductor current slope in the initial phase, when input and output are connected directly. As shown in Fig 2.7, 
when the slope of inductor current in initial phase is greater than 0 , converter operates in boost mode. And converter operates in buck mode when the inductor current in initial phase is decreasing. It means that when $V_{I N}$ is close to $V_{O U T}$, the mode switches between buck and boost modes automatically depending on the slope of the inductor current. Because the same control circuit is used for the whole range in both modes, the smooth transition is achieved. In this method, no input voltage sensing is needed for the mode selection. Smooth transition is accomplished by switching between buck and boost mode. In addition, the hysteresis ripple bounds can reportedly be adjusted so as to produce a variable switching frequency that indirectly increases efficiency, Also there are almost no switching activity when $V_{I N}$ is close to $V_{O U T}$, so that the switching losses are further reduced during the transition. However, this control method has variable switching frequency depending on the operation point, which is not optimal for EMI sensitive applications.

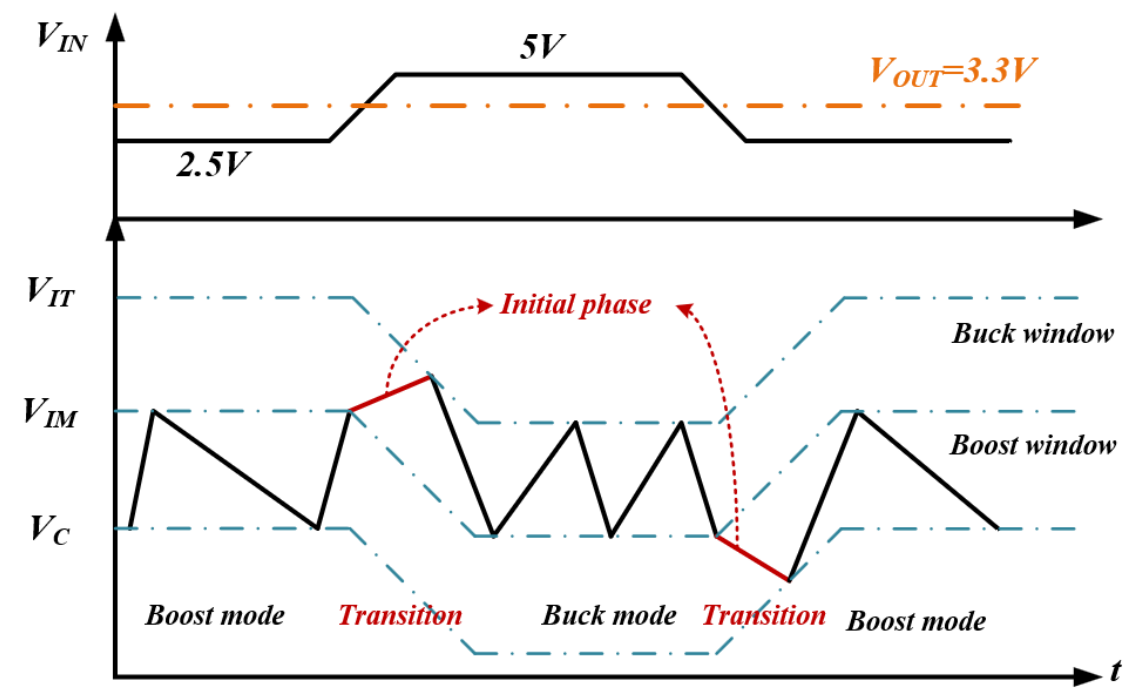

Figure 2.7: Hysteretic control mode transition between buck and boost mode 


\section{Chapter 3}

\section{Low-frequency ripple-shaping mode}

\subsection{Introduction}

The ripple-shaping concept has been proposed for the use in high power converters [26]. There, through alteration of the switching sequence, converter inductor current waveforms are modified, compared to their conventional shapes, to minimize the RMS current values, and therefore, reduce conduction losses. As shown in Fig 3.2, by reshaping the inductor current waveform from triangle to trapezoid, average inductor current reduces to almost half of buck-boost operation. In addition, inductor current ripple can be reduced drastically. The key features and advantages of RS mode are explored in the following subsections. Section 3.2 investigates the RS mode, including the switching sequence, conversion ratio and inductor current characteristics. The converter operates in such manner that both step-up and step-down conversion ratios can be achieved, which could be regarded as a large hysteretic band for the transition between buck and boost mode. Additionally, the inductor current level is reduced to almost the same as load current and inductor current ripple only depends on the minimum on-time of the power switches and input/output voltage. Section 3.3 introduces dual-frequency modulation concept for the NIBB converter to enhance the advantages of RS mode. The RS mode is operated at relatively low switching frequency compare to buck or boost mode. So that not only conduction losses is reduced but the switching losses too. Section 3.4 explains the transition issues related to the introduction of the RS mode. And practical solutions to achieve seamless transition between buck or boost mode to RS mode is introduced. Then practical a implementation is shown and effectiveness of the controller will be demonstrated by experimental results. Efficiency improvements are shown mathematically by analyzing the losses breakdown of RS mode and confirmed by experimental results. 


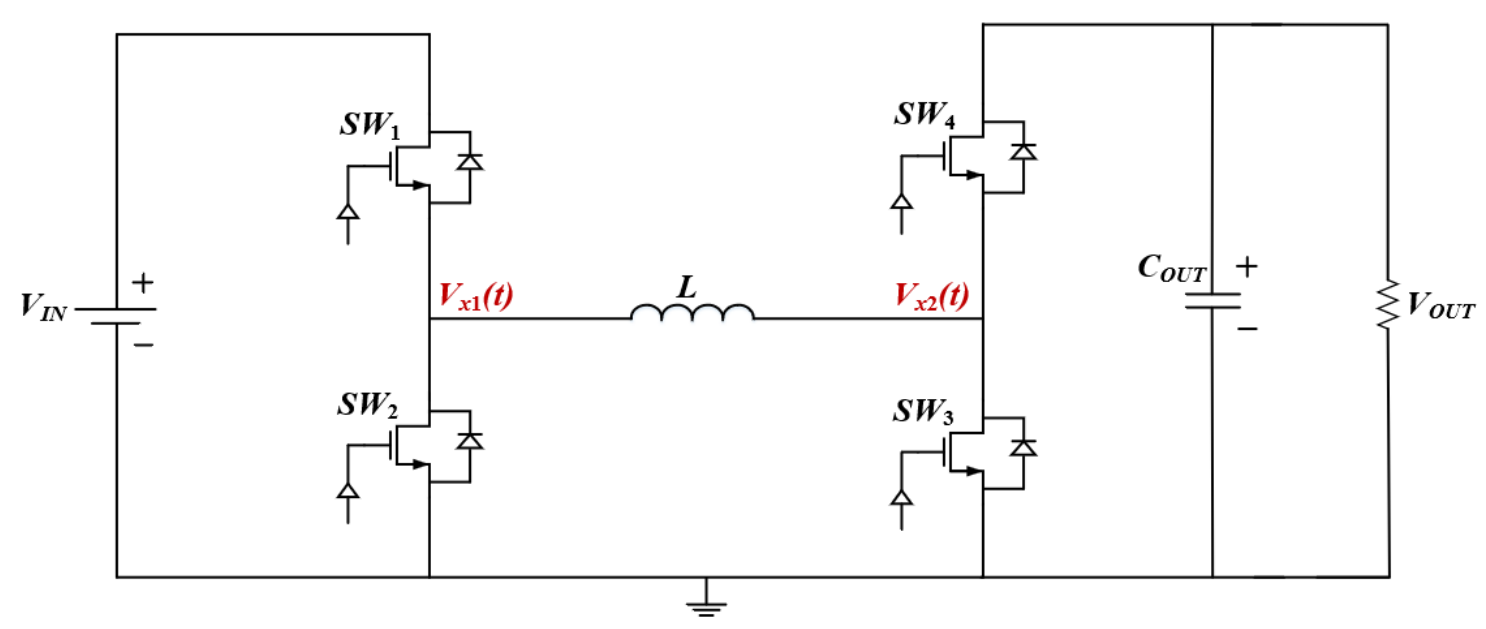

Figure 3.1: Non-inverting buck-boost converter

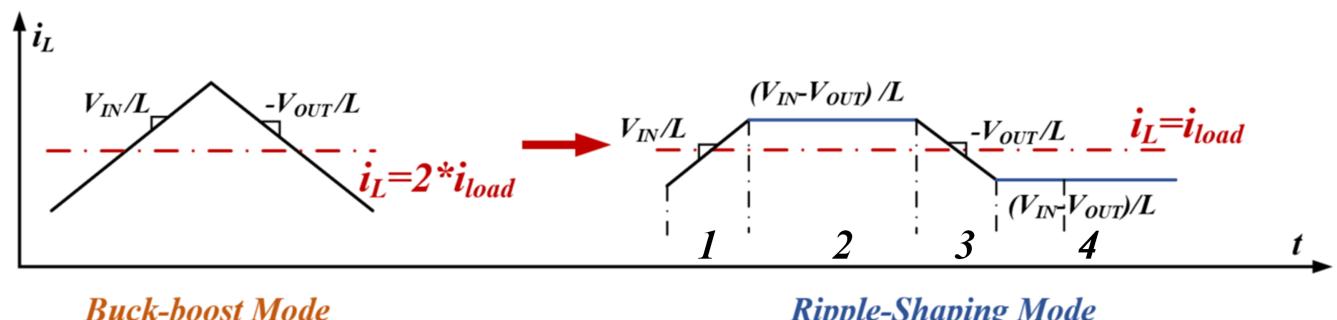

Figure 3.2: Inductor current waveform comparison between buck-boost mode and RS mode

\subsection{Principle of Operation}

The current shaping switching sequence and corresponding waveforms in RS mode for the conventional NIBB Fig 3.1 when $V_{I N}$ is equal to $V_{O U T}$, are shown in Fig 3.3. There are four intervals in each switching cycle. The inductor is charging through $V_{1}$ and $V_{3}$ in first subinterval, where the slope of the inductor current is $V_{I N} / \mathrm{L}$. In the second and fourth subintervals, input and output are connected directly through $S W_{1}$ and $S W_{4}$, where the slope of the inductor current is $\left(V_{I N}-V_{O U T}\right) / \mathrm{L}$. And the inductor is discharged through $S W_{2}$ and $S W_{4}$ in subinterval 3. In Fig $3.3 D_{1}^{*} T_{S}$ defines the transistor $S W_{1}$ on time and $D_{2}{ }^{*} T_{S}$ is the on time of $S W_{4}$. RS mode can achieve both step-down and step-up conversion ratio. Additionally, the inductor current level is reduced to almost the same value as the load current and the inductor current ripple only depends on the minimum on time of the power switches and input/output voltages. As shown in the 
inductor current waveform in Fig 3.3, when $V_{I N}$ is equal to $V_{O U T}$, the current ripple is independent on the switching frequency. Therefore, $\mathrm{RS}$ mode is not only capable of reducing conduction losses drastically but switching frequency and switching losses can be reduced with minimal impact on the inductor current ripple. Utilizing these key features of RS mode operating in the range of $V_{I N}$ close to $V_{O U T}$, trade-offs between switching and conduction losses can be optimized.

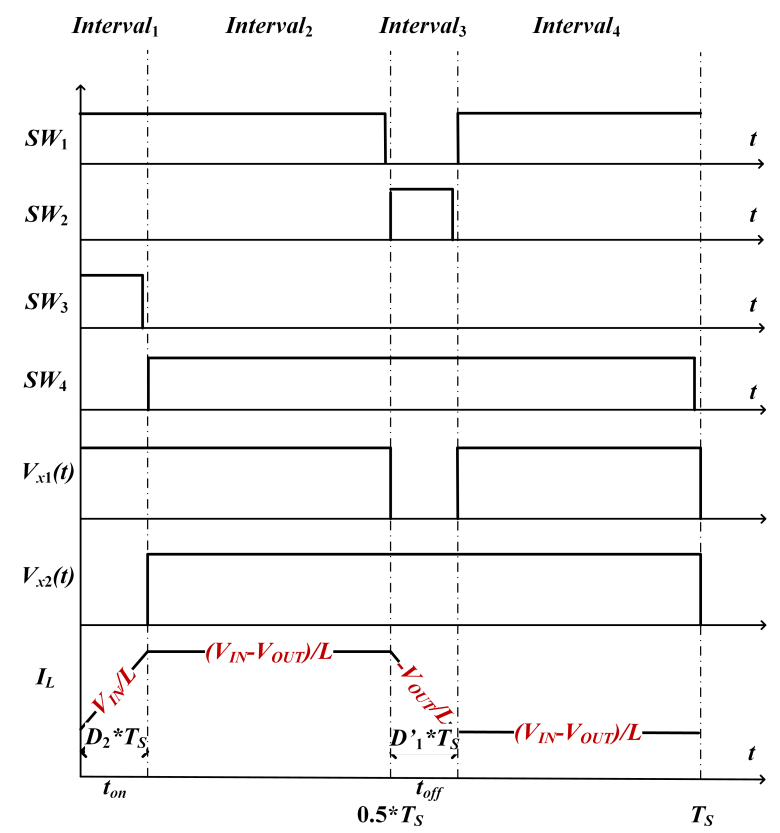

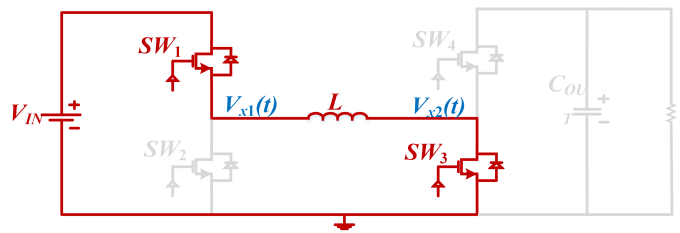

(a) Subinterval 1 inductor charging phase

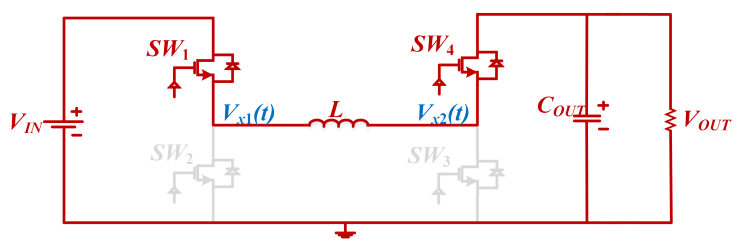

(b) Subinterval 2,4 direct connection between input and output

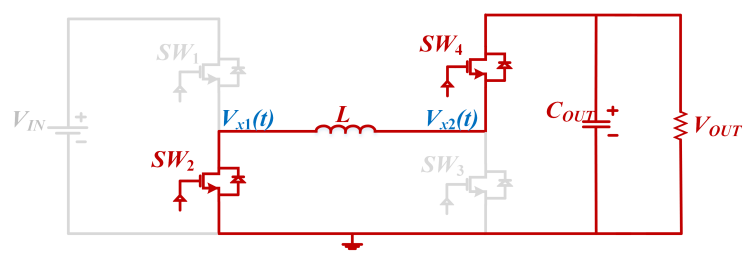

(c) Subinterval 3 inductor discharging phase

Figure 3.3: Key waveforms of ripple-shaping mode including gating signals, switching node voltage, inductor current(left) and operation subintervals(right)

The regulation of the output voltage and mode switching is performed by a digital average current programmed mode (CPM) controller, which will be described in subsection 3.6 .

\subsubsection{Conversion Ratio of RS mode}

Utilizing volt-second balance [17], the conversion ratio of the RS mode can be calculated as:

$$
M\left(D_{1}, D_{2}\right)_{R S}=\frac{D_{1}}{1-D_{2}}
$$

where, the conversion ratios of buck and boost mode are:

$$
M\left(D_{1}\right)_{b u c k}=D_{1},
$$




$$
M\left(D_{2}\right)_{b o o s t}=\frac{1}{1-D_{2}}
$$

where $D_{1}$ is the ratio between the turn on time of $S W_{1}$ and period $T_{S}$, while $D_{2}$ is the ratio between turn on time of $S W_{3}$ and period $T_{S}$.

This equation shows that the conversion raito of RS mode is a function of both $D_{1}$ and $D_{2}$. When turn on time of the $S W_{1}$ is smaller than the turn off time of the $S W_{3}$, the converter operates in step down (Fig 3.4a). Buck mode is the case where $S W_{3}$ is off and $D_{2}$ is 0 . In RS step down mode, the turn on time of $S W_{3}$ is fixed as a minimum value to keep the lowest inductor RMS current. The inductor current ripple analysis will be shown in the next subsection. Likewise, while in RS step up mode, the turn on time of $S W_{1}$ is fixed at a maximum value so that the minimum turn on time of $S W_{3}$ can be obtained for the required conversion ratio.

Fig 3.4 show the inductor current waveform during the transition between buck/boost modes to RS step down/up mode, where discrepancy between input and output voltage is not negligible. The positive and negative slope of the inductor current when input and output are directly connected exist confirms the RS mode maintains the ability to provide both step down and step up functions.

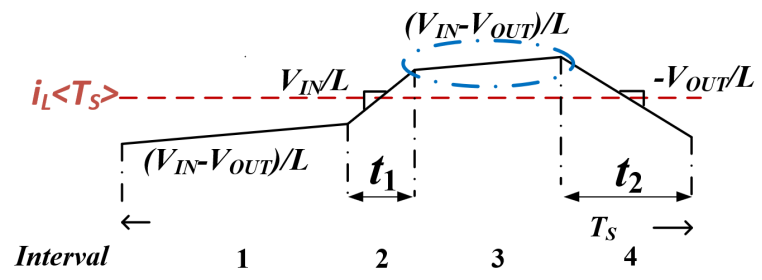

(a) RS step-down mode

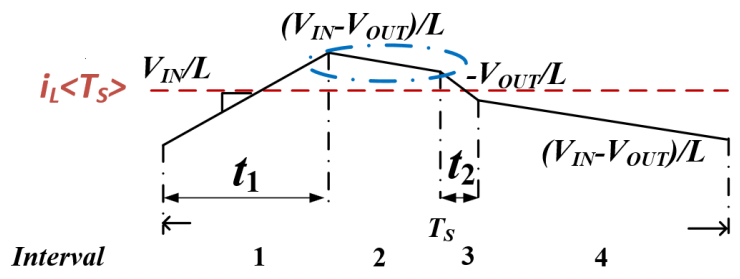

(b) RS step-up mode

Figure 3.4: Inductor current waveforms in step-down mode and step-up mode (a) shows the step-down case where slope of initial phase and $t_{2}$ is greater than $t_{1}$. (b) shows the step-up case where slope of initial phase is negative and $t_{2}$ is smaller than $t_{1}$

\subsubsection{Inductor current level and ripple reduction}

According to capacitor-charge balance [17], the average inductor currents in three modes are:

$$
I_{L(a v e) R S}=I_{L o a d} \cdot \frac{1}{1-\frac{t_{\text {on }} W_{3}}{T_{S}}}
$$




$$
\begin{gathered}
I_{L(\text { ave }) \text { buck }}=I_{\text {Load }}, \text { and } \\
I_{L(\text { ave }) \text { boost }}=I_{\text {Load }} \cdot \frac{1}{1-D_{2}}
\end{gathered}
$$

$I_{L(\text { ave }) R S}$ is the average inductor current in $\mathrm{RS}$ mode, $I_{L(\text { ave)buck }}$ is the average inductor current in buck mode and $I_{L(a v e) b o o s t}$ is the average inductor current in boost mode.

As shown in the equations and Fig 3.5, in RS mode, the duration of interval that energy only transfer to inductor is kept as minimum. Therefore, the average inductor current can be lower than that of buck-boost operation, where the duty ratio is 0.5 (coressponding for $V_{I N}=V_{O U T}$ ) and the inductor current is twice the load current when $V_{I N}$ equals to $V_{\text {OUT }}$. In fact, the RS mode has almost the same average current as a buck. This can be achieved by maintaining a constant small turn on time of $S W_{3}$, and by reducing the switching frequency.

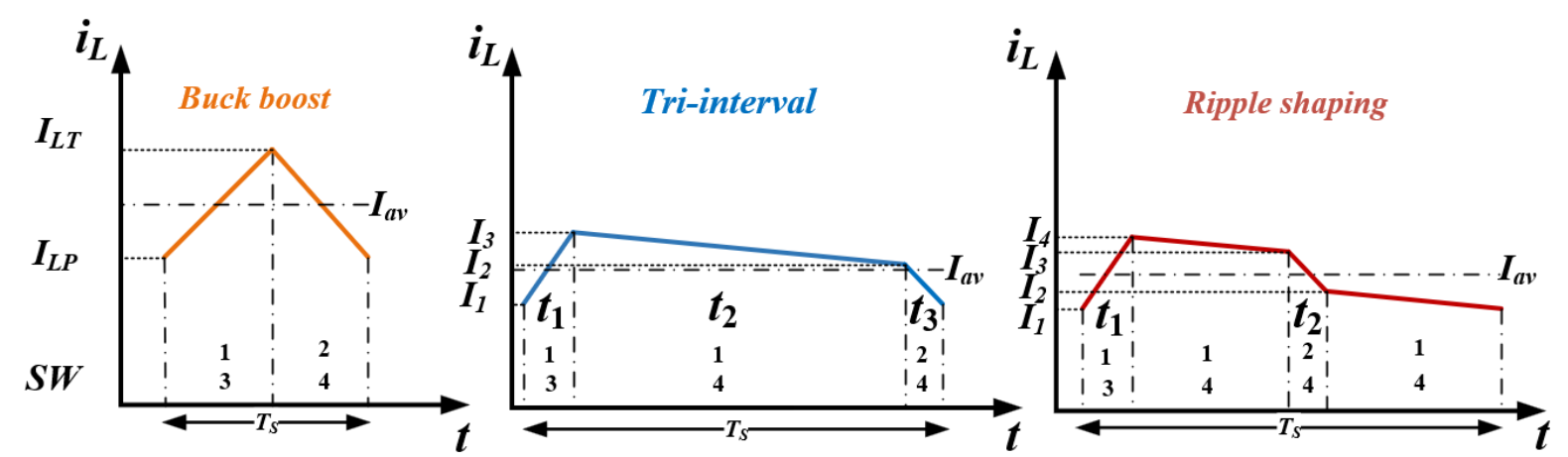

Figure 3.5: Inductor current in buck boost mode, tri-interval mode and Ripple-shaping mode

Inductor RMS current in RS mode is reduced compared to other state-of-the-art solutions. In Fig 3.5, comparisons are made among RS mode, buck boost mode and triinterval operation that is used in a state of the art commercial product[27]. According to the definition of RMS current i.e.:

$$
i_{r m s}^{2}=\frac{\int_{0}^{T_{S}} i_{L}^{2}(t) d t}{T_{S}}
$$

The RMS current of buck-boost mode is: 


$$
I_{\text {Lrmsbuckboost }}=\sqrt{\frac{I_{L P}^{2}+I_{L T}^{2}+I_{L P} \times I_{L T}}{3}}=\sqrt{I_{a v}^{2}+\frac{\Delta I_{P-P}^{2}}{12}}
$$

The RMS current of tri-interval mode is:

$$
I_{\text {LrmsTri-interval }}=\sqrt{\frac{I_{1}^{2}+I_{2}^{2}+I_{1} \times I_{2}}{3} \times \frac{t_{1}}{T_{S}}+\frac{I_{3}^{2}+I_{2}^{2}+I_{2} \times I_{3}}{3} \times \frac{t_{2}}{T_{S}}+\frac{I_{1}^{2}+I_{3}^{2}+I_{1} \times I_{3}}{3} \times \frac{t_{3}}{T_{S}}}
$$

The RMS current of RS mode is:

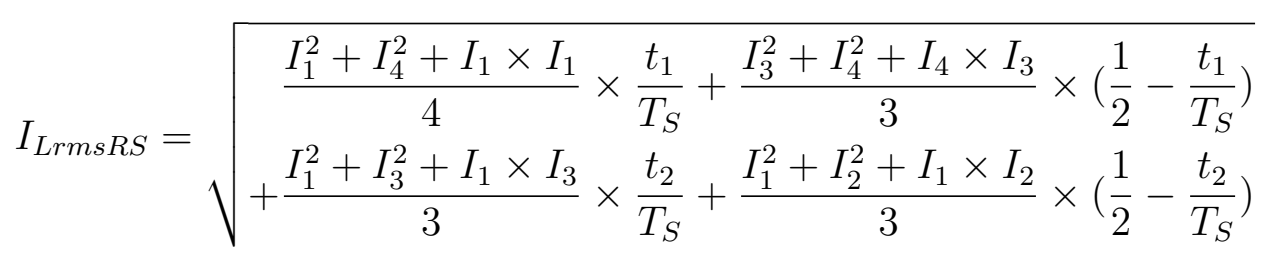

where $t_{1}, t_{2}, t_{3}, I_{1}, I_{2}, I_{3}$ and $I_{4}$ are labeled in Fig 3.5.

Similar to RS step down and step up modes mentioned before, in tri-interval operation, when $t_{1}$ is greater than $t_{3}$, the converter operates at step up mode. And when $t_{1}$ is smaller than $t_{3}$, the converter operates at step down mode.

A comparisons of normalized RMS currents among buck-boost mode, tri-interval mode and RS mode during the transition are shown in the following figures. Aforementioned tri-interval operation [12] and RS mode both has the ability of reducing inductor RMS current compared to buck-boost mode. When the conversion ratio equals 1 , the RS mode reduced to $46 \%$ and tri-interval operation reduced to $44 \%$. By looking at the equations and figures, RS mode has the lowest RMS inductor current among all the methods. 


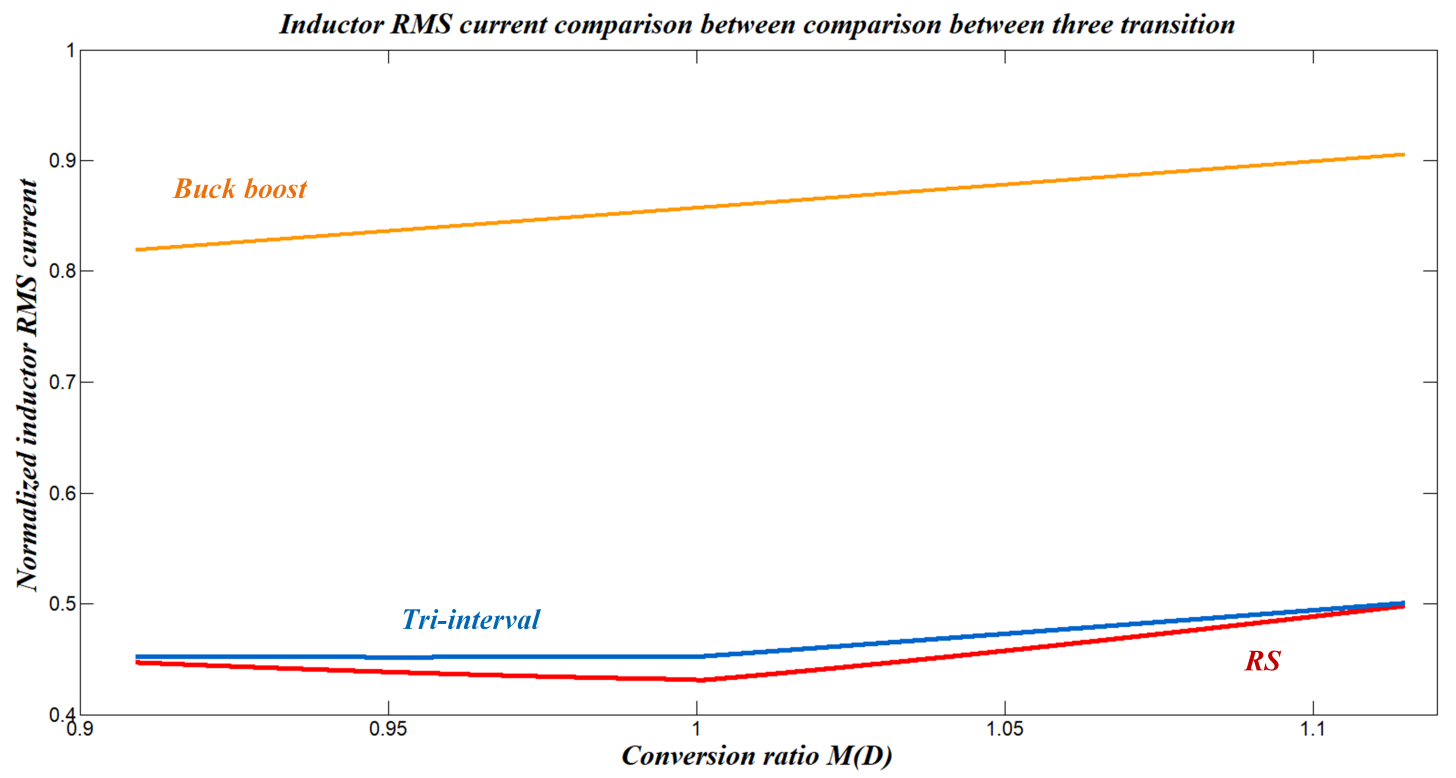

Figure 3.6: Normalized RMS current comparison in the dead zone

\subsection{Dual-Frequency Modulation}

As discussed in the previous subsection, lower average inductor current can be obtained by lowering the switching frequency. Furthermore, it can be noticed that for $V_{I N}$ equals to $V_{O U T}$, the ripple amplitude can be kept at approximately the same level while reducing the switching frequency as shown in Fig 3.7. Therefore, to utilize this advantage, in the introduced controller, dual-frequency operation is utilized. In RS mode, the converter operates at a lower switching frequency than that of the buck and boost mode. The lower frequency is selected based on the peak inductor current and ripple limitation and/or to be the same as an existing low-frequency mode of operation for a given converter that, in the targeted applications, is usually used for light load operating conditions. Examples include various schemes where the converter operates in the discontinuous conduction (DCM) at a much lower frequency than during heavy and medium load conditions. The energy stored in an inductor is $W_{L}=1 / 2 \cdot L \cdot I_{\text {peak }}{ }^{2}$ and depends on the inductance and peak inductor current. Therefore, no larger inductor than the existing design requirements for the boost condition is needed for the low frequency operation in RS mode. The frequency change between buck/boost mode to RS mode is a step change if compares to other state-of-the-art solutions [11], so RS mode is well-suited for EMI sensitive application like portable devices where two frequency operation is oftenly used. 


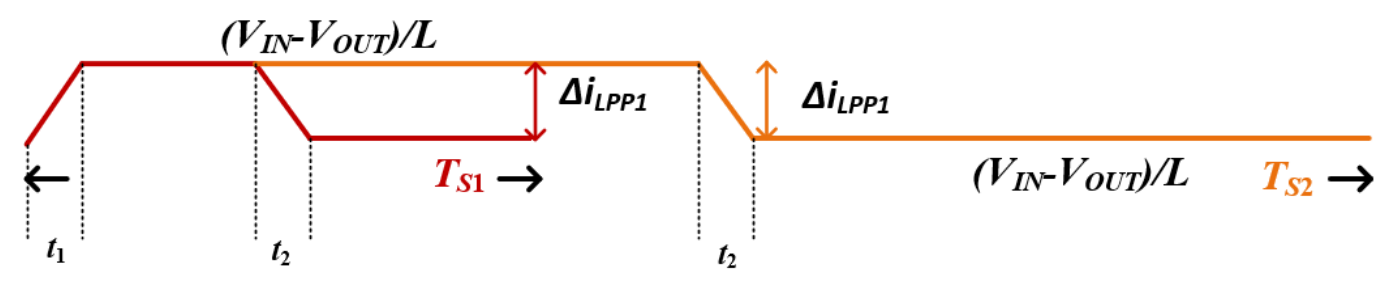

Figure 3.7: Inductor current waveform in RS mode under different frequency

\subsection{Seamless Transition}

Introduction of the RS mode with dual-frequency modulation requires both switching sequence and frequency change when mode switches between buck or boost mode and RS mode. As it will be shown later, thanks to the flexibility of digital control, extra transition technique to the original control loops is relatively easy to add. Mode selection is based on comparison between input voltage and reference voltages. Hysteretic band is added at the boundary of the transtion point.

\subsubsection{Mode Selection}

The mode selection logic chooses the most preferable operating mode according to the input voltage and output voltage with hysteretic band considered. RS control logic is bypassed when converter operates in buck or boost mode. When the converter operates in RS step-up mode, the turn off time of $S W_{1}$ is fixed at a minimum value. The turn on time of $S W_{3}$ and $S W_{4}$ are regulated by the compensator. While the converter is in RS step-down mode, the turn on time of $S W_{3}$ is fixed at a minimum value and turn on time of $S W_{1}$ and $S W_{2}$ are calculated by the compensator. Minimum turn on time depends on the rise and fall time of the power switches. The buck switch pair $S W_{1,2}$ and boost switch pair $S W_{3,4}$ work sequentially with a 180 degree phase shift to shape the current into a trapezoid-like form, as shown in Fig 3.3. The period of DPWM is extended in RS mode, which decreases the effective switching frequency of the converter. Counter based DPWM is used in this case.

A hysteretic band logic is used to avoid the mode toggling during transitions. The operation of the hysteretic band logic can be described with the help of the diagram of Fig 3.8. The transition point for the mode change depends on the trending of the $V_{I N}$. 
For example, when $V_{I N}$ is increasing, the mode switches at the larger value of the band (the right side of a shaded portion on the diagram). Mode changes from boost to RS step up when $V_{I N}$ is larger than $V_{2}$. While when $V_{I N}$ is decreasing, operation mode changes from RS to boost when $V_{I N}$ is smaller than $V_{1}$. It should be noted that this type of hysteretic transition is by its nature much less susceptible to mode toggling than those used in other solutions, since the converter does not change its voltage conversion function, so the problem is practically eliminated.

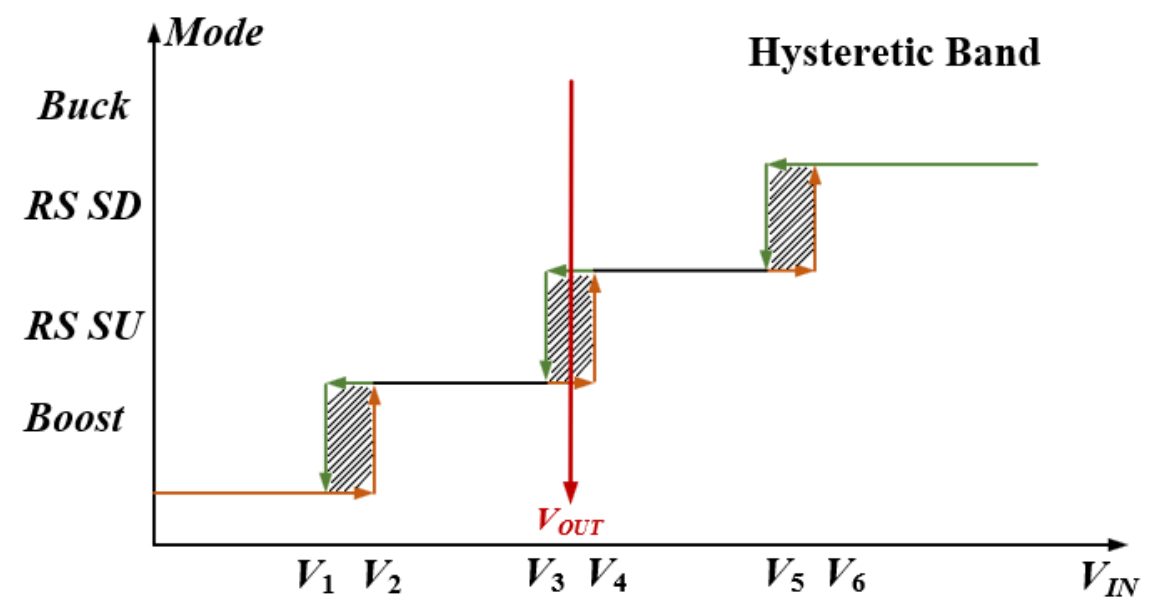

Figure 3.8: Hysteretic Band

\subsubsection{Compensator Reset}

In the RS mode, one of the buck or boost duty ratios are locked. In RS step-down mode, boost duty ratio $D_{2}$ is locked at the minimal value to reduce the inductor RMS current. $D_{1}$ is calculated by compensator, to regulate the output voltage. Likewise, in RS step-up mode, buck duty ratio $D_{1}$ is locked at the maximum value and $D_{2}$ is controlled. However, when converter switches between step up and step down modes, duty ratio changes immediately. This sudden change can't be accomplished with a PID compensator without causing large variations in the inductor current and the output voltage. When mode changes from RS step-down to RS step-up, the duty ratio needs to change from the maximum value to minimal value. And instant overshoot for inductor current and output voltage would occur. Similarly, a large drop would happen when mode switches from RS step-up to RS step-down. It would take a compensator hundreds cycle to recovery. In order to address this problem, compensator reset is implemented when the mode switches between RS step-down and step-up as shown in Fig 3.10. When the mode transition signal is detected. The compensator is bypassed for one cycle. And 
the output of the compensator is reset to the corresponding value according to the mode, i.e. maximum value for RS step-down or minimum value for $\mathrm{RS}$ step-up. These values are stored in a register and fed back to the compensator. Therefore, for the next cycle, the new duty ratio will be calculated based on error signal and reset value. This technique reduces the burden of the compensator and makes mode transition smoother. The output voltage overshoots or undershoots are practically eliminated avoiding the damage to the converter. Because the targeted duty ratio for the next mode is the locked value from previous mode, the reset values are known and seamless transition is achieved.

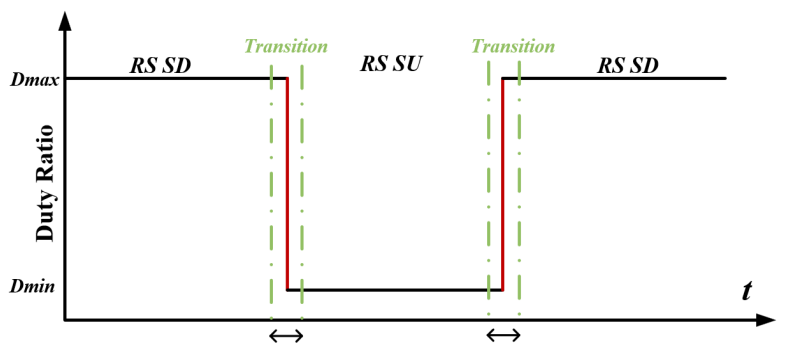

(a) Transition between RS SU mode and RS SD mode with reset

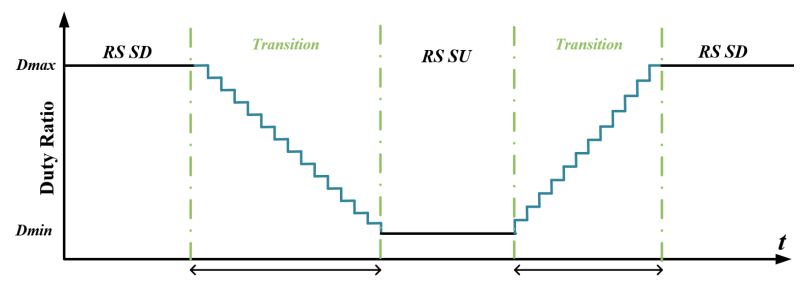

(b) Transition between RS SU mode and RS SD mode without reset

Figure 3.9: Duty ratio change with compensator reset(a) and without compensator reset(b) 


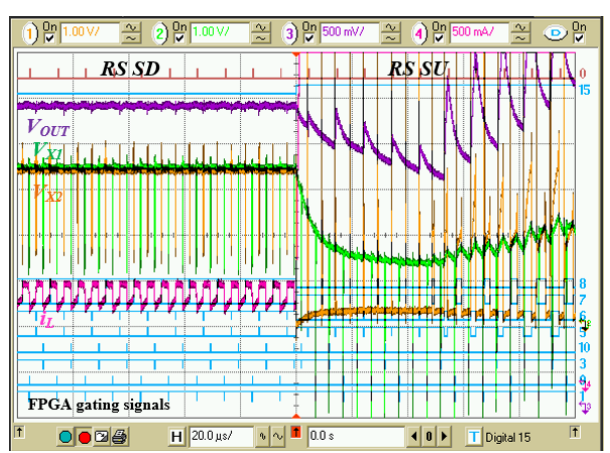

(a) Transition from RS Step-down mode and RS Step-Up mode without reset

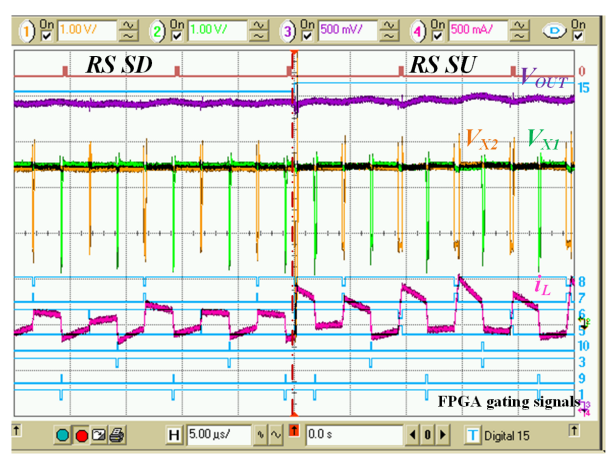

(c) Transition from RS Step-down mode and RS Step-Up mode with reset

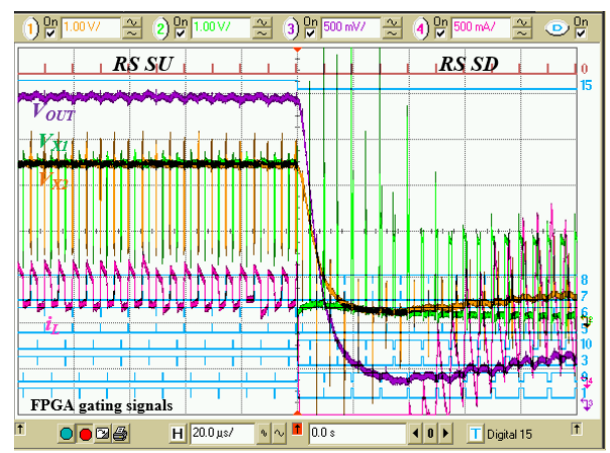

(b) Transition from RS Step-Up mode and RS Step-down mode without reset

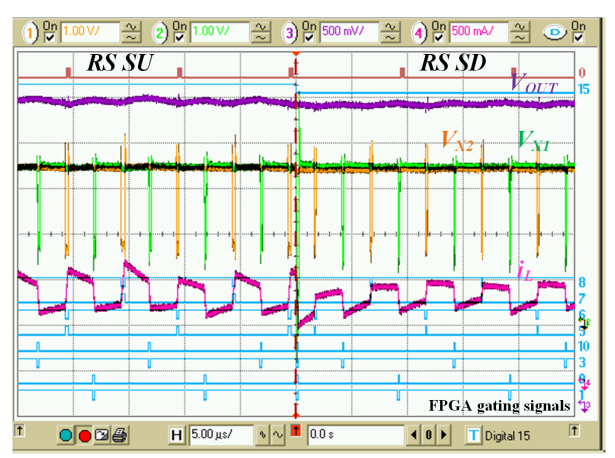

(d) Transition from RS Step-Up mode and RS Step-down mode with reset

Figure 3.10: Experimental results verify the effectiveness of duty ratio reset technique

\subsubsection{Frequency Scaling}

For the transition between buck or boost to RS shaping mode, both frequency and switching pattern are changed. For the switching sequence, when mode changes from buck/boost to RS step-down/up mode, the duty ratio of $S W_{3} / S W_{1}$ changes from 0/1 to the minimum/maximum achievable value that depends on the switching frequency. For the low-frequency operation, the minimum achievable duty ratio in the boost is smaller and maximum achievable duty ratio in the buck is larger. For example, when minimum turn on time of the power switches is $50 \mathrm{~ns}$ while the switching frequency of RS is 200 $\mathrm{kHz}$, the minimum locked duty ratio is $1 \%$ and for $2 \mathrm{MHz}$ that value would be $10 \%$. This allows the minimum impacts on the duty ratio change on the controlled switches. Under the assumption that input voltage changes gradually, which is applicable in battery powered application. We can assume that at the transition point, conversion ratio is the 
same. In order to keep simplicity of the controller, frequency scaling is implemented by extending the turn on time of the controlled switch and keeping the same duty ratio from the previous cycle, to accommodate the frequency change. As shown in Fig 3.11 and Fig $4.17 \mathrm{a}$, duty ratio is kept the same while the conversion ratio is extended as if dead zone does not exist. Transition results will be shown in subsection 3.6.2.

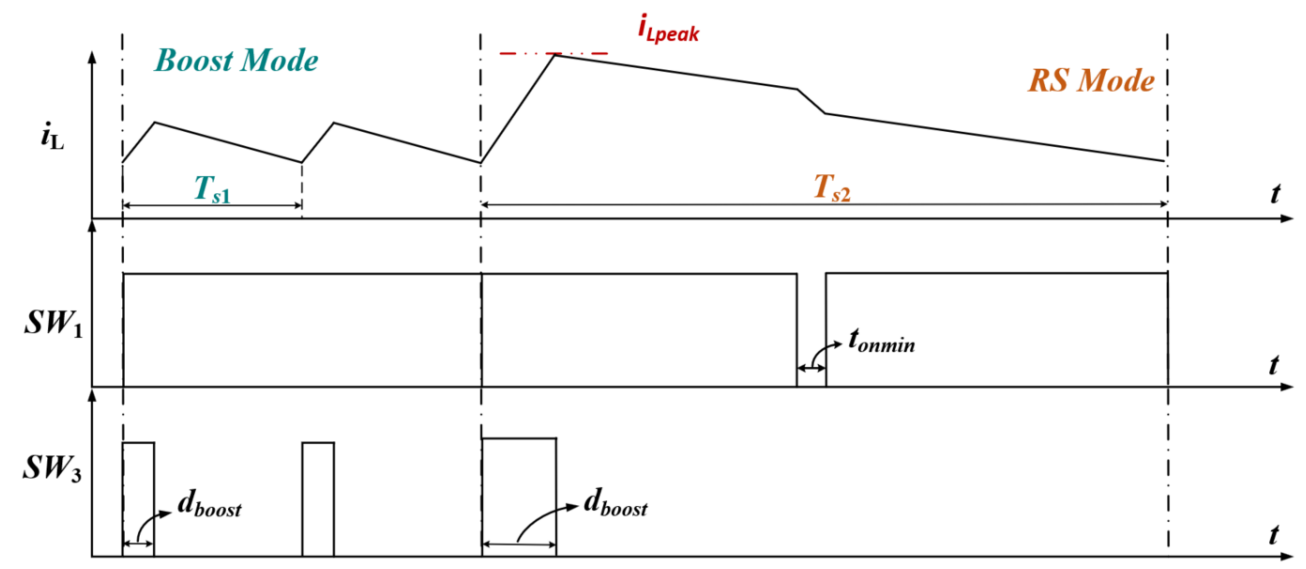

Figure 3.11: Inductor current waveform change from boost mode to RS mode

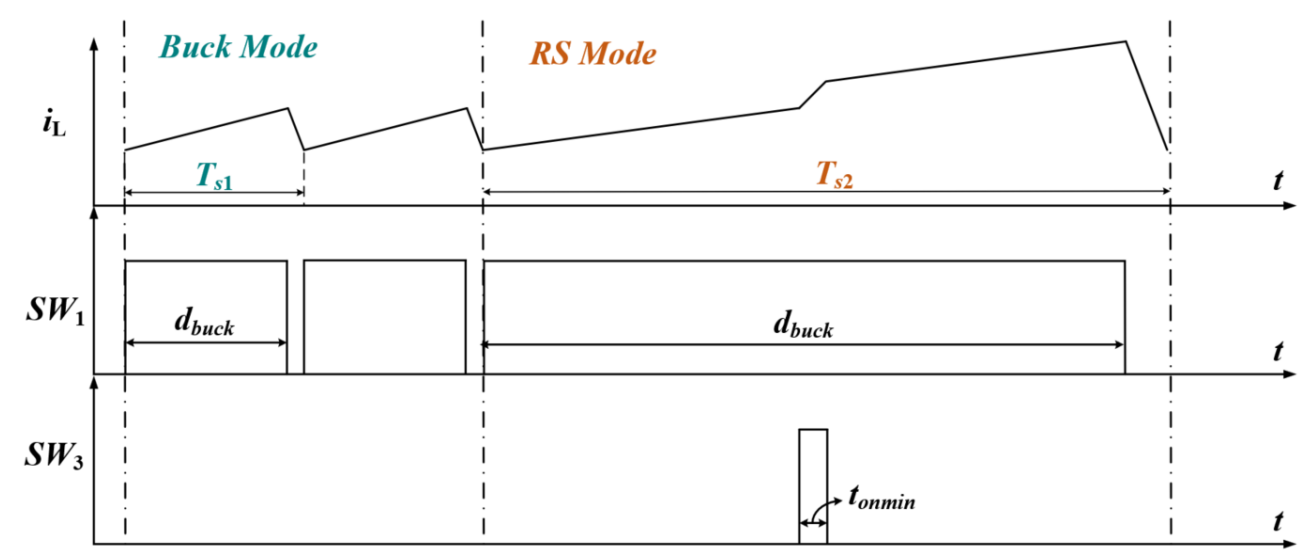

Figure 3.12: Inductor current waveform change from buck mode to RS mode

\subsection{Practical Implementation}

\subsubsection{Average CPM}

A practical implementation of the ripple-shaping logic control is shown in Fig 3.13. The controller is a modification of a conventional average current programmed (CPM) controller containing an outer output voltage loop and an inner current regulation loop, 
both of which are digital. Three ADCs are used for sensing the input voltage, the output voltage, and the inductor current. A digital equivalent of the attenuated output voltage $H^{*} V_{O U T}[n]$ is compared with the reference $V_{R E F}[1-6]$ shown in the Fig 3.13 . The voltage compensator then takes the resulting error $e_{v}[n]$ and sets the inductor current reference $i_{R E F}[n]$. The current loop compensator compares $i_{R E F}[n]$ to a digital equivalent of the inductor current and, accordingly, provides the duty ratio control variable $d[n]$ to the digital pulse width modulator (DPWM). In this case, the controller also contains Mode Selection Logic that decides the mode of operation based on the converter operating conditions. In addition, mode transition block has compensator reset and frequency scaling to achieve the seamless transition between buck or boost and RS modes.

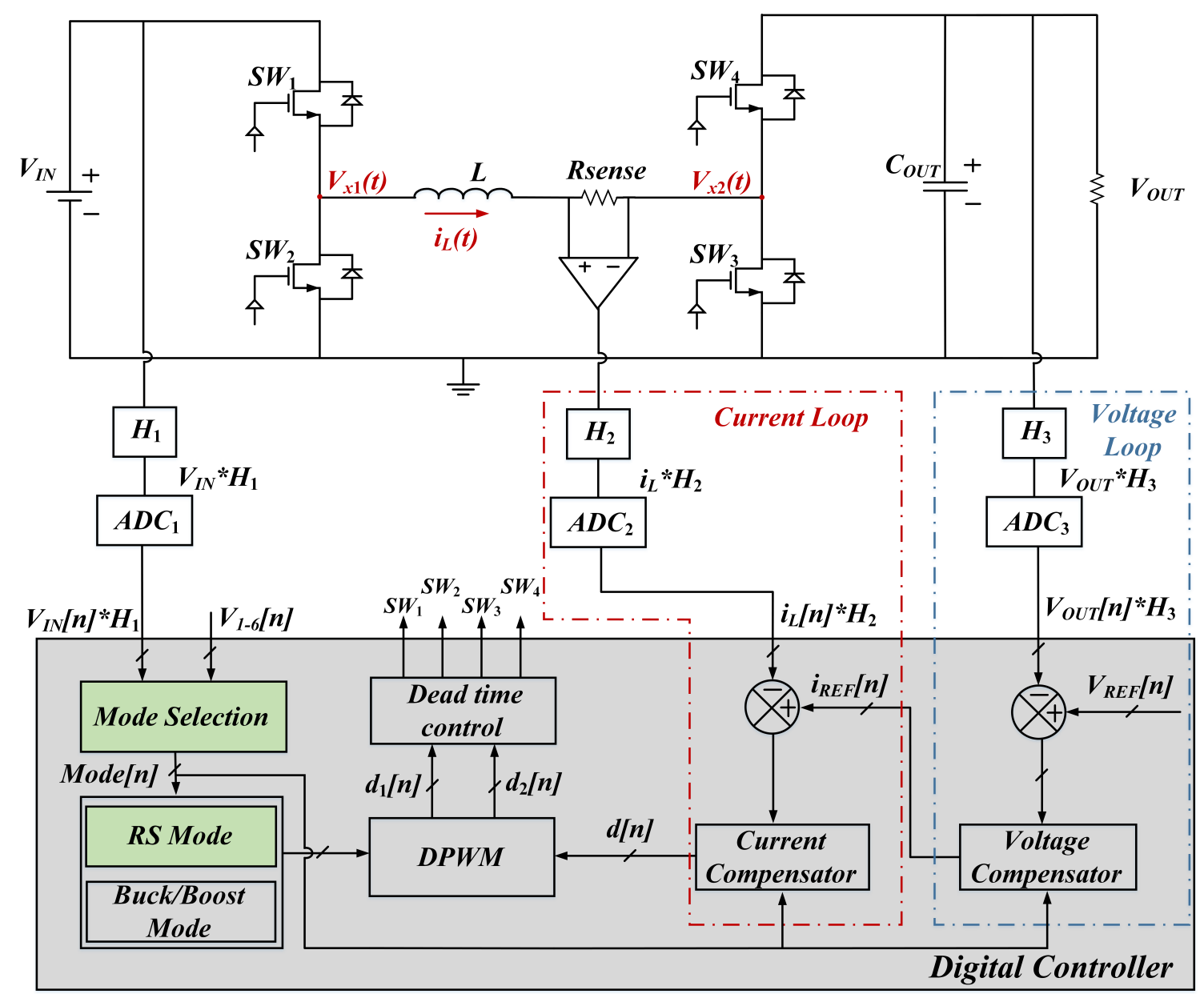

Figure 3.13: Non-inverting buck-boost converter regulated with a low-frequency rippleshaping controller, implemented through digital average current mode controller 


\subsubsection{Experimental Results}

An experimental prototype has been built to verify the operation of the introduced lowfrequency ripple shaping based buck-boost controller. The controller was created using an FPGA-based evaluation board and the parameters of a custom-made discrete power stage are shown in Table 3.1. Steady state operation of each mode and smooth transition between each mode will be presented. Furthermore, using the same power stage, the performance of the new controller are compared to the performances of the controllers of two commercial state of the art solutions [25, 27]. Fig 3.14 to Fig 3.17 show the results of experimental measurements.

Table 3.1: Converter Parameters

\begin{tabular}{|c|c|}
\hline Parameters & Value \\
\hline$V_{I N}$ & $1.8 \mathrm{~V}-5 \mathrm{~V}$ \\
\hline$V_{\text {OUT }}$ & $3.3 \mathrm{~V}$ \\
\hline$I_{\text {OUT }}$ & $0.5 \mathrm{~A}-3 \mathrm{~A}$ \\
\hline$f_{\text {Sbuck } / \text { boost }}$ & $1 \mathrm{MHz}$ \\
\hline$f_{\text {SRS }}$ & $200 \mathrm{kHz}$ \\
\hline$L$ & $2.2 \mu \mathrm{H}$ \\
\hline$C_{\text {OUT }}$ & $45 \mu \mathrm{F}$ \\
\hline
\end{tabular}

Fig 3.14 show four distinctive modes of operation namely buck, boost, RS step down and RS step up, respectively. Fig 3.14a and 3.14b show the buck and boost mode operation respectively. Fig $3.14 \mathrm{c}$ and $3.14 \mathrm{e}$ show the RS mode near the transition point where the discrepancy between input and output voltage is ineligible. The switching frequency in RS mode is $1 / 5$ of buck/boost mode. And there are four subintervals in one switching cycle. The inductor current ripple is relatively large but the peak inductor current is still smaller than the peak current in the boost operation due to the low average inductor current. Therefore, no larger inductor is needed. However, for the switching losses point of view, power processing efficiency is increased. Fig 3.14d and 3.14f present the case where voltage across the inductor is almost 0 . It can be seen that, even though the converter operates at 5 times lower switching frequency in RS mode compared to buck and boost modes $(200 \mathrm{kHz}$ vs $1 \mathrm{MHz})$, the current ripples are still comparable. However, from the power processing efficiency point of view large advantages are gained. 


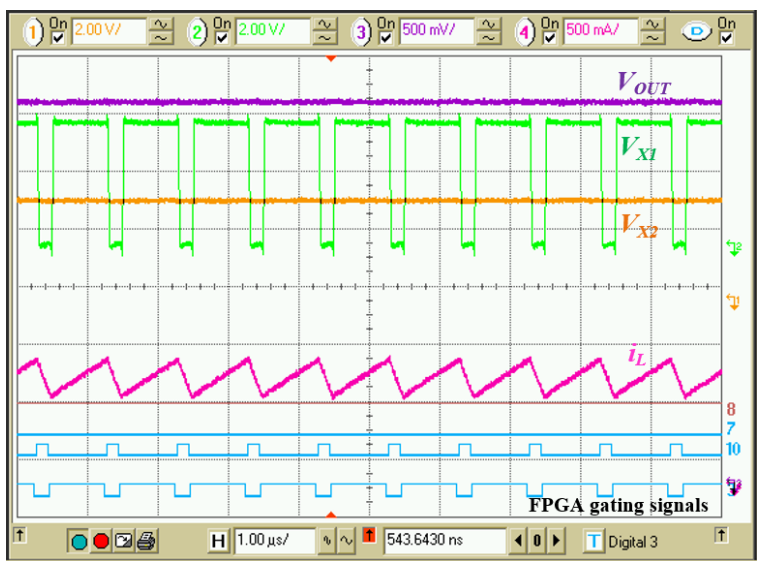

(a) Buck Mode

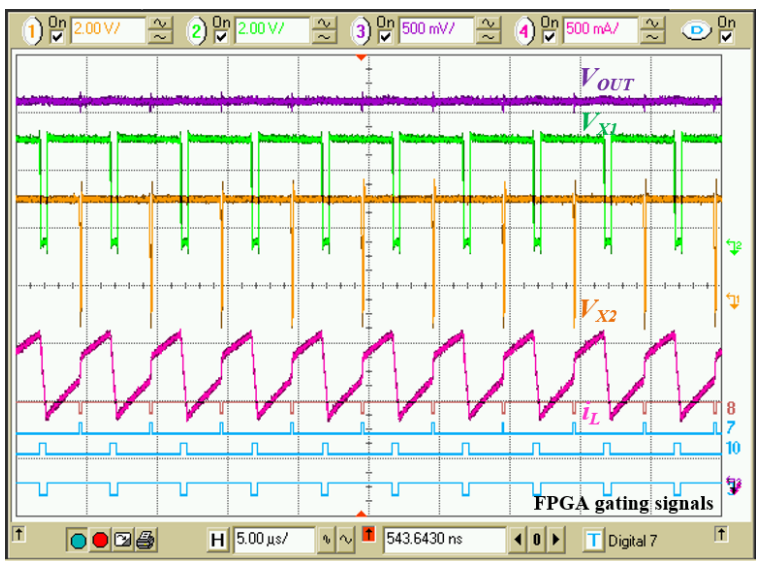

(c) RS step-down mode

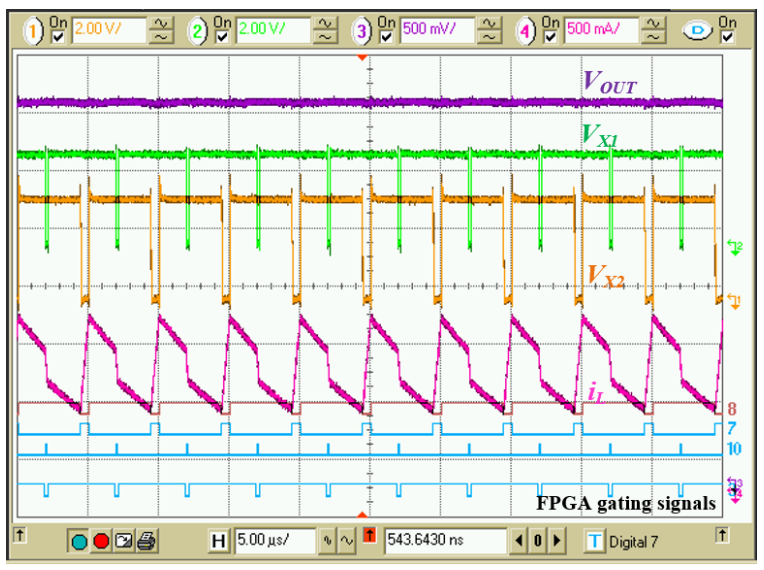

(e) RS step-up mode

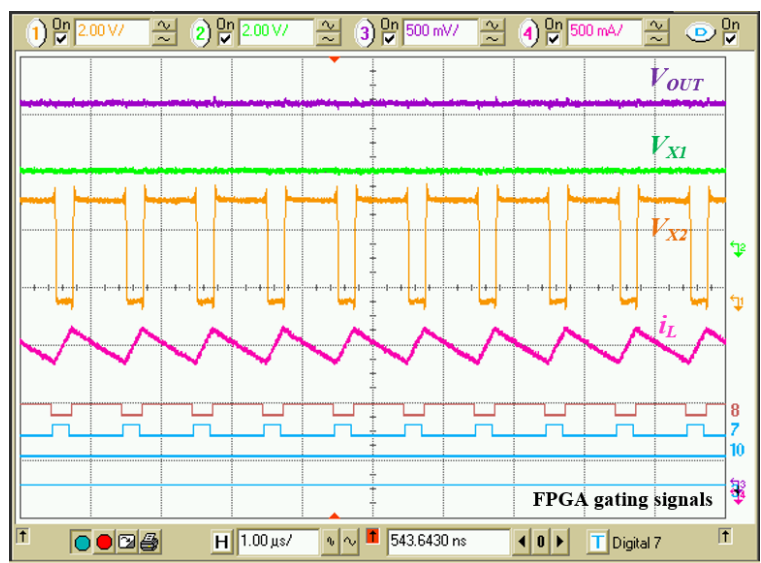

(b) Boost Mode

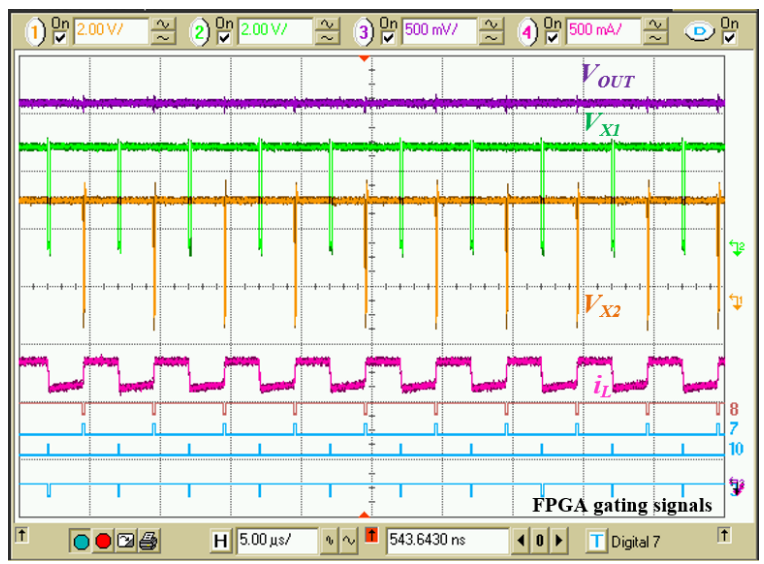

(d) RS step-down mode

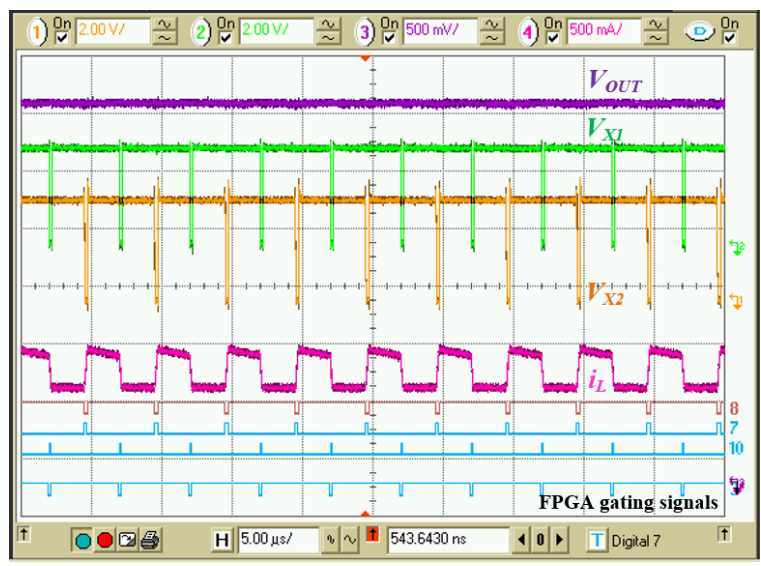

(f) RS step-up mode

Figure 3.14: Steady state operation in (a) buck (b) boost (c,d) RS step-down (e,f) RS step-up mode; For all the waveforms: Ch.1 (Orange): $2 \mathrm{~V} /$ div output switching node voltage; Ch.2 (Green): $2 \mathrm{~V} /$ div input switching node voltage; Ch.3 (Purple): $500 \mathrm{mV} /$ div output voltage; Ch.4 (Pink): $500 \mathrm{mV} /$ div inductor current; 
Fig 3.15 show mode transition between buck mode, boost mode, RS step down mode and RS step up mode. Fig 4.17b, 3.15d, 3.15f, 3.15h, 3.15j, 3.15l are zoomed in version of the corresponding transitions shown in the left column. The results confirm fast transient and stable operation of all modes. Zoomed results show, when mode switches from buck/boost to RS mode, shape of the inductor current changes from triangular to trapezoidal with switching frequency reduced $1 / 5$ of the previous mode. Small variation at inductor current and output voltage can be seen due to the approximation on the duty ratio at the transition point. The variation at output voltage varies according to the compensator design. Generally, the deviation is under reasonable range around 3\%, which justifies the approximation that at the transition point duty ratio is about the same. As described in section 3.4.3, only turn on time of the switches is extended with the frequency change. As voltage across inductor getting smaller, the inductor current ripple decreases to the minimum value shown in Fig 3.15i and 3.15l. No overshoot or undershoot occurs when converter switches the mode between step-up and step-down. No mode toggling has been observed. These experimental results confirm functionality and effectivness of the introduced mode transition technique. 


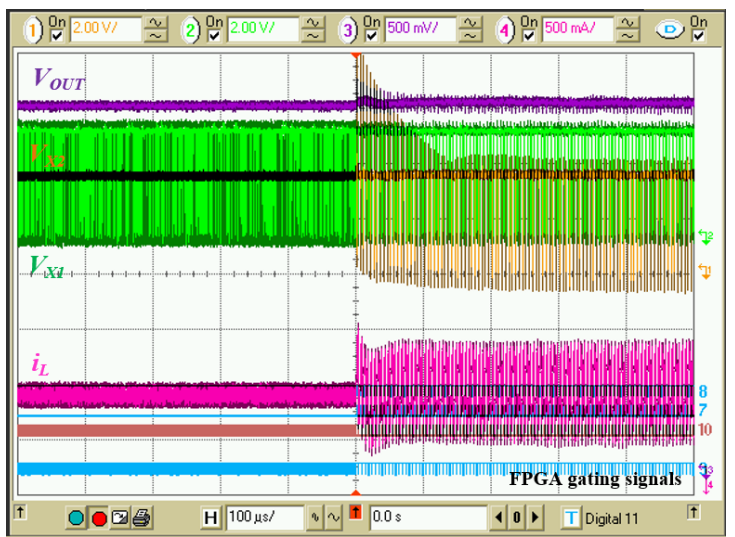

(a) Transition from buck mode to RS SD mode

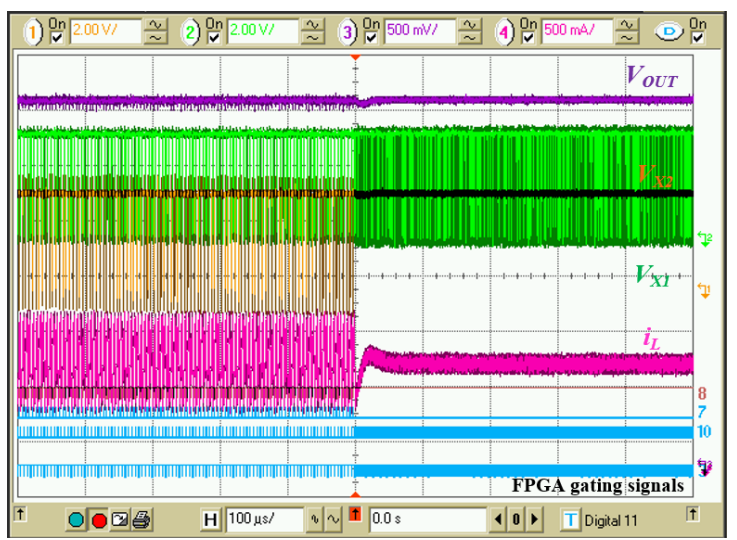

(c) Transition from RS SD mode to buck mode

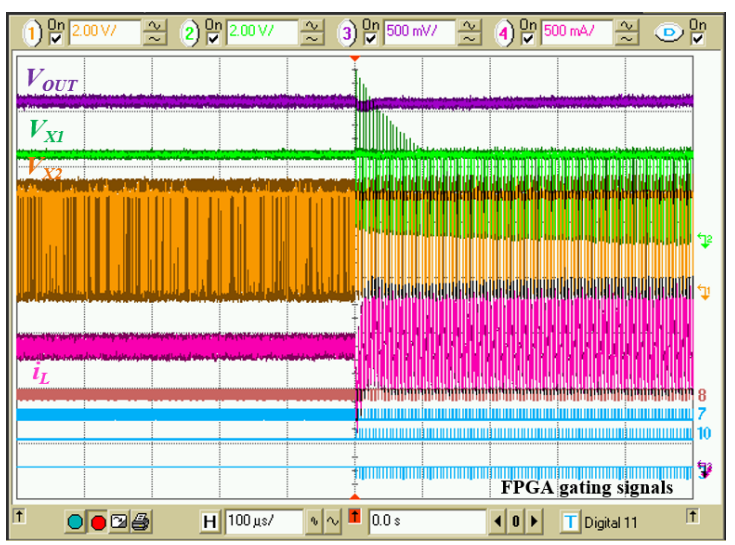

(e) Transition from boost mode to RS SU mode

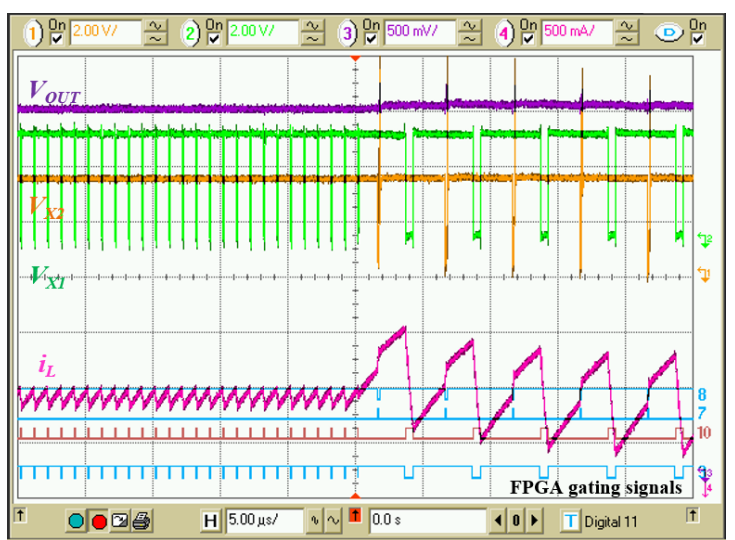

(b) Transition from buck mode to RS SD mode zoomed

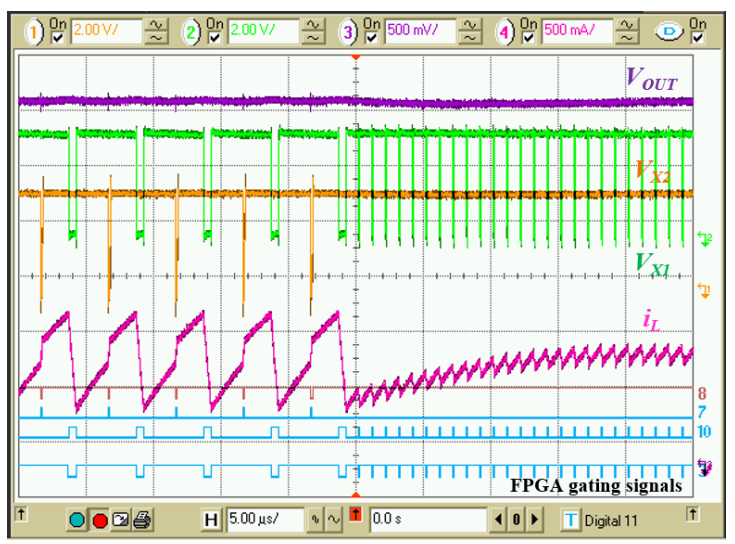

(d) Transition from RS SD mode to buck mode zoomed in

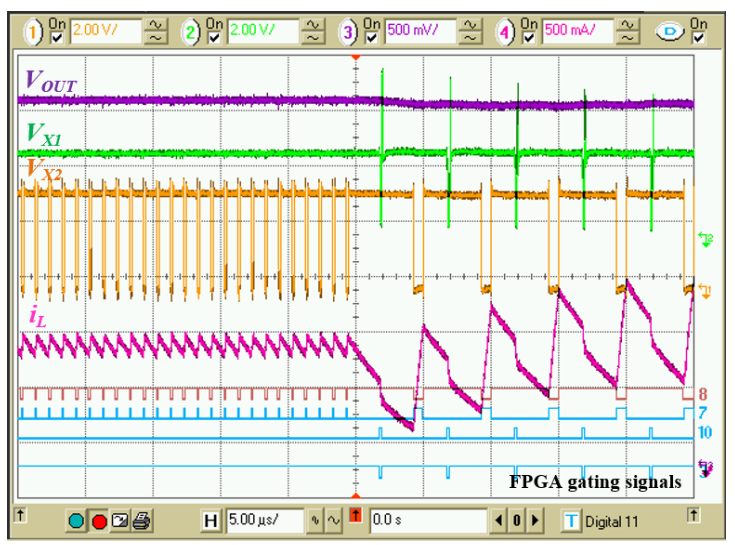

(f) Transition from boost mode to RS SU mode zoomed in 


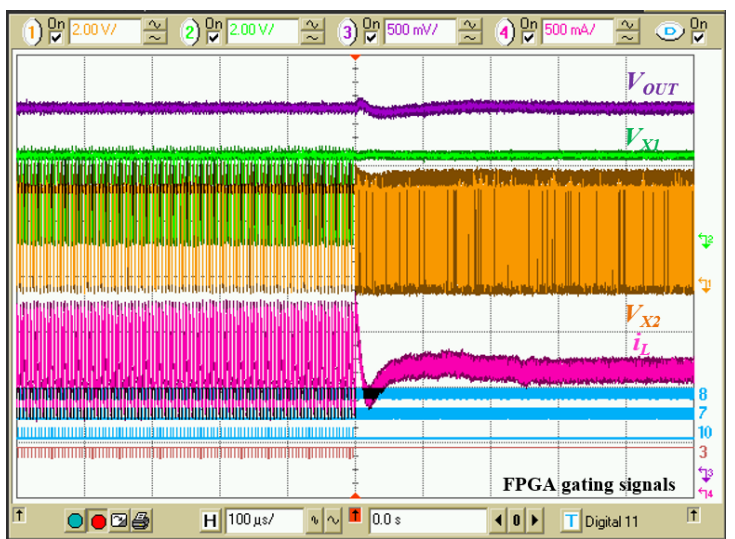

(g) Transition from RS SU mode to boost mode

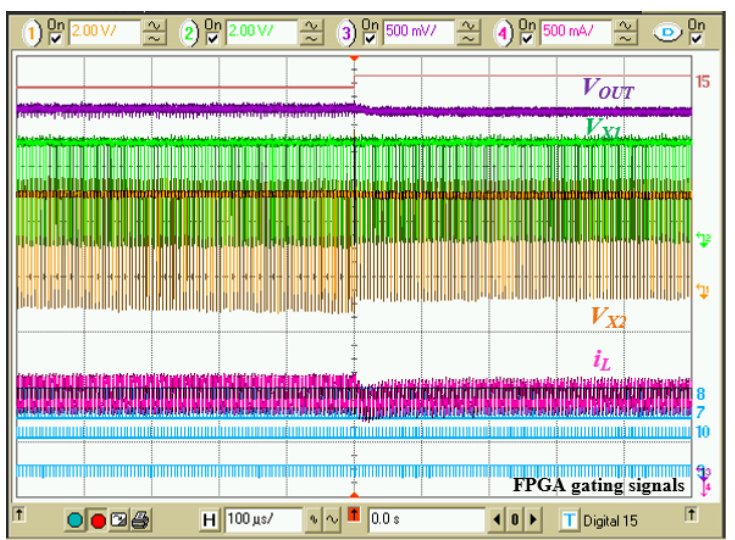

(i) Transition from RS SD mode to RS SU mode

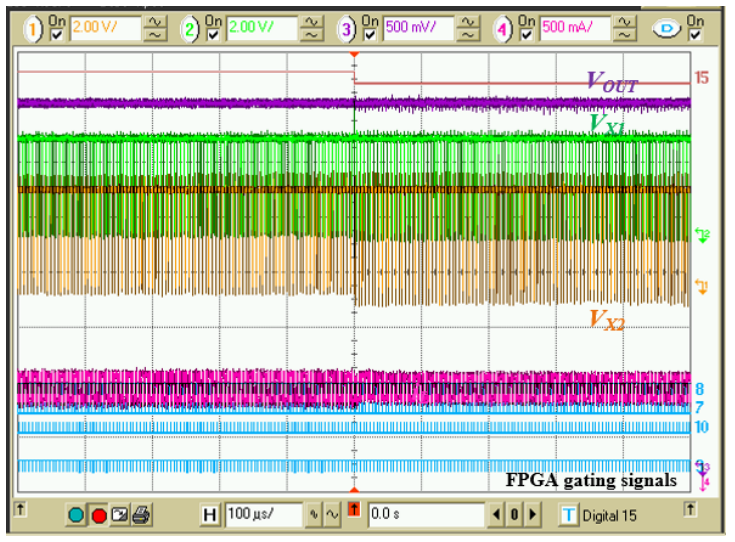

(k) Transition from RS SU mode to RS SD mode

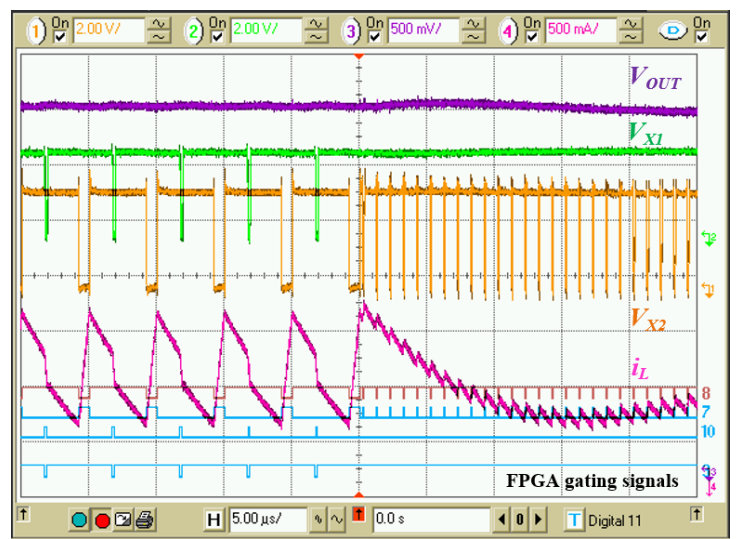

(h) Transition from RS SU mode to boost mode zoomed in

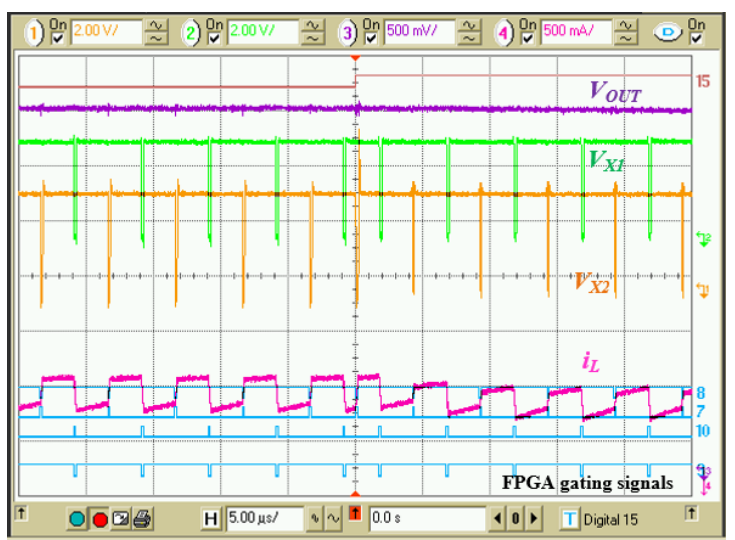

(j) Transition from RS SD mode to RS SU mode zoomed in

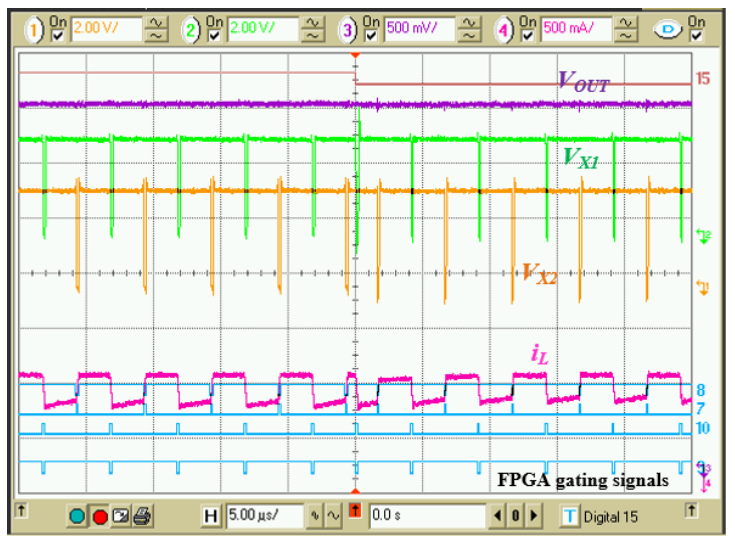

(1) Transition from RS SU mode to RS SD mode zoomed in

Figure 3.15: Mode transition between (a,b,c,d) buck and RS SD (e,f,g,h) boost and RS SU (i,j,k,l) RS SD and RS SU; For all the waveforms: Ch.1 (Orange): $2 \mathrm{~V} /$ div output switching node voltage; Ch.2 (Green): $2 \mathrm{~V} /$ div input switching node voltage; Ch.3 (Purple): $500 \mathrm{mV} /$ div output voltage; Ch.4 (Pink): $500 \mathrm{mV} /$ div inductor current; 
Losses breakdown comparison between RS mode and other state of the art solutions [25, 27] are shown in Fig 3.16. The lighter color of each bar stands for switching losses and darker color of each bar stands for conduction losses. The results are shown for the case when the conversion ratio is 1 , for several load current values ranging from $0.5 \mathrm{~A}$ to $3 \mathrm{~A}$. As shown in the figure, RS mode has the lowest switching losses due to the low switching frequency. Mixed-mode method and tri-interval operation method has the same switching losses. In terms of conduction losses, RS and the other two commercial products are comparable. Based on the aforementioned analysis, compare to buck-boost mode, other methods have the ability of reducing RMS inductor current so as the conduction losses. While RS mode has the lowest RMS current due to the trapezoidal waveform and low frequency operation.

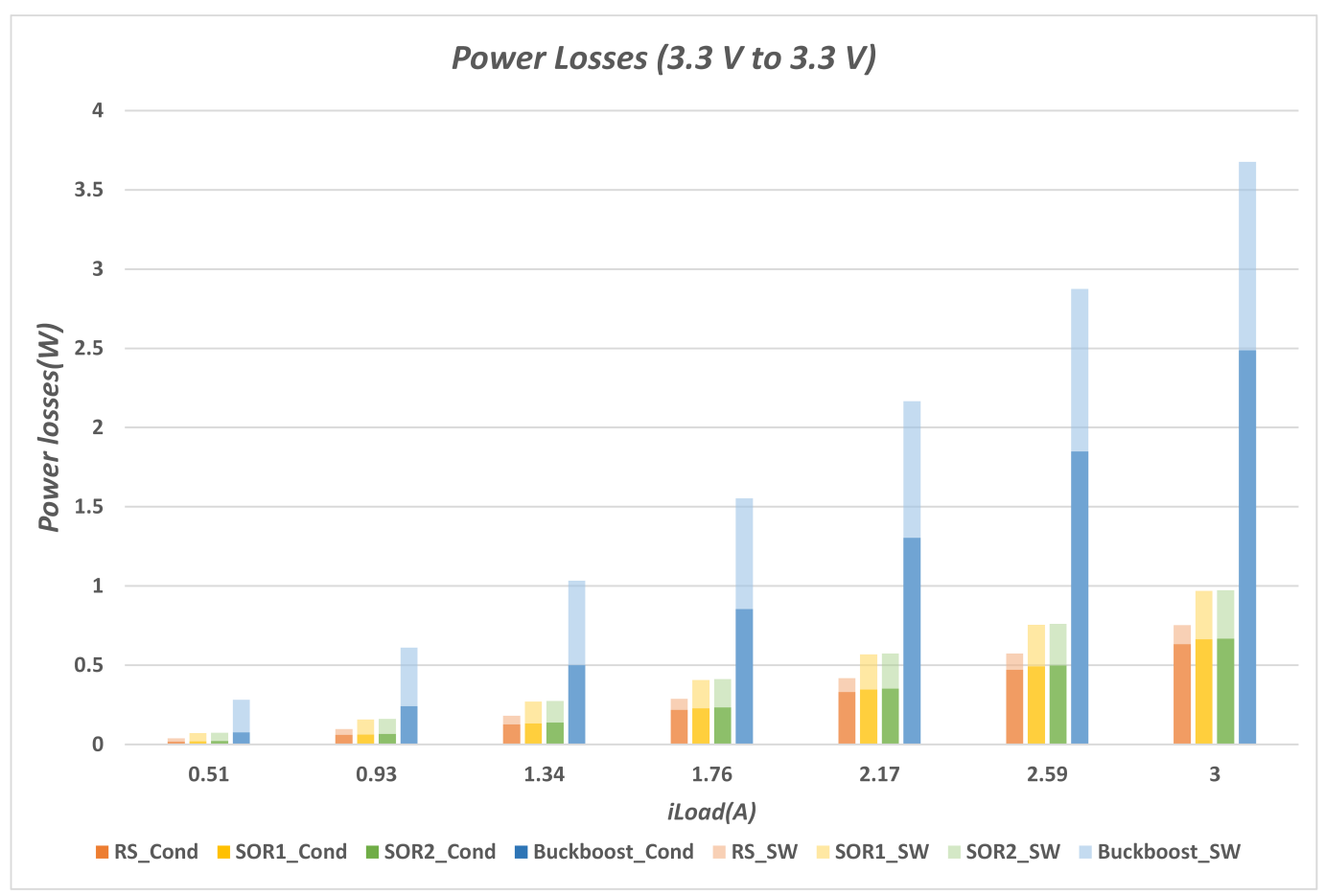

Figure 3.16: Power losses comparison

Fig 3.17 shows efficiency measurement comparison between the introduced ripple shaping controller and two state-of-the-art commercial solutions [25, 27] for load currents varying from $0.5 \mathrm{~A}$ to $3 \mathrm{~A}$, for one-to-one conversion ratio. The controller of [27] is using tri-interval operation and [25] is using mixed-mode operation (both of which described in section 2.1) where when $V_{I N}$ equals to $V_{O U T}$, steady state operation is buck mode and boost mode operating sequentially. Efficiency of RS mode and other two state of art are comparable in terms of efficiency improvements. According to the analysis shown before, 
trapezoidal waveform has lower RMS inductor current compared to tri-interval operation. On top of that, lower switching frequency reduce switching losses significantly. Aforementioned factors result in higher power processing efficiency improvements among other control methods. Results show that the low frequency ripple-shaping control improves efficiency by about $4 \%$ at lighter loads and by $6 \%$ at heavy loads.

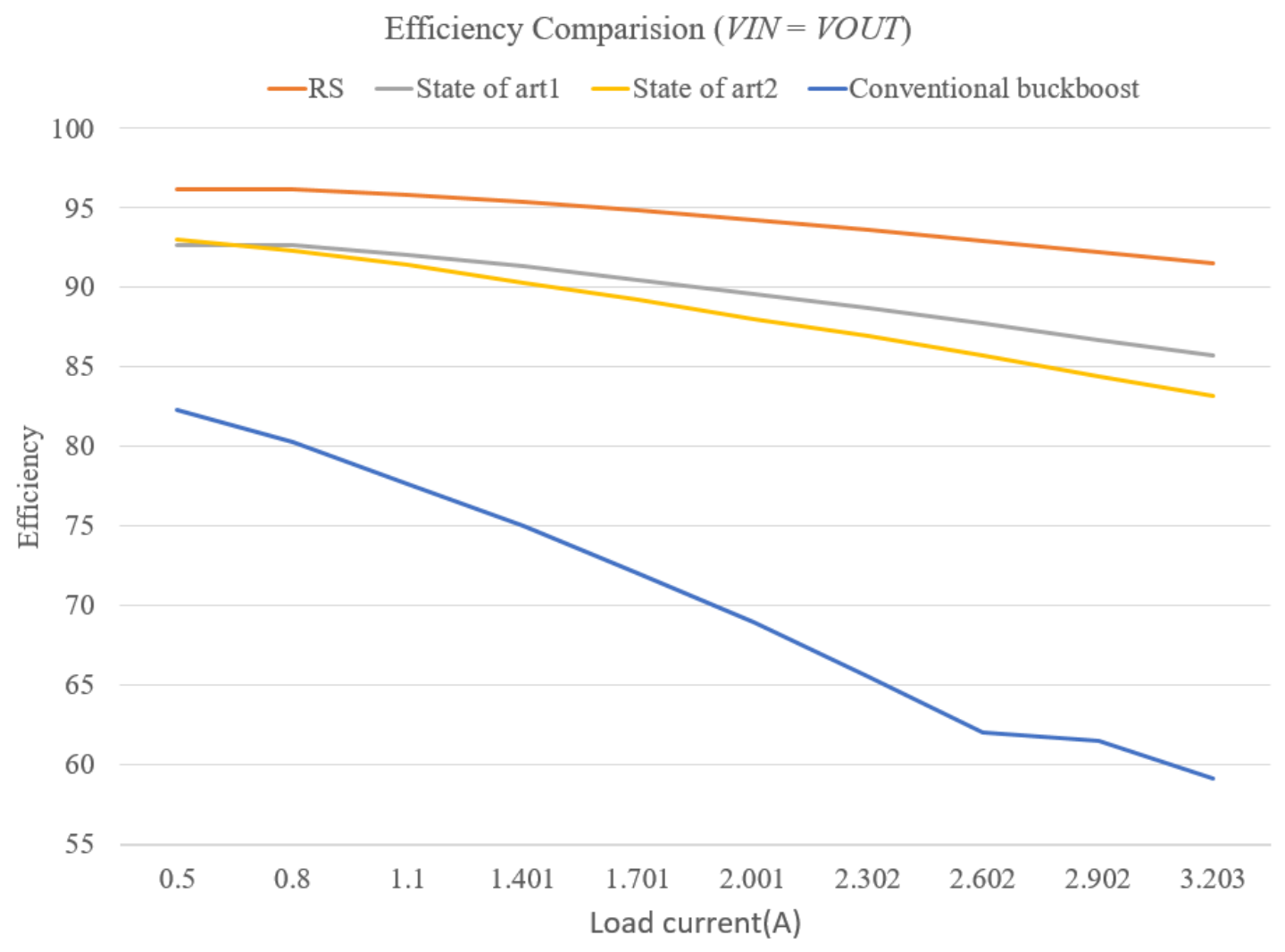

Figure 3.17: Efficiency comparison between RS and other state of art

\subsection{Conclusion}

A RS based dual-frequency controller is introduced for smooth operation of NIBB converters in the transition region between buck and boost modes. The method practically eliminates mode transition problems existing in commonly use solutions and, at the same time, improves power processing efficiency for conversion ratios close to one-to-one. These advantages are achieved by operating in a single mode that allows both step-up and step-down function and providing operation at low frequency with low current ripple. In addition, the mode transition methods that minimizes voltage and current transients during mode changes is presented. Seamless transition between buck or boost mode 
and RS mode is achieved. Furthermore, practical implementation of the introduced controller in digital manner is shown. Experimental results verify the effectiveness of the mode transition strategy as well as the efficiency improvements of the introduced RS mode. 


\section{Chapter 4}

\section{Multilevel NIBB}

\subsection{Introduction}

The inductors of NIBB, which dominants the overall converter size, are a large obstacle in further reduction of size and/or increasing of capabilities of portable devices. The inductors occupy relatively large volume that could potentially be also shared with some emerging functional blocks.

Operating the converter at higher frequencies would allow smaller inductor [17]. However, it would come with the penalty of increased switching core losses. The multi-level buck converter has been utilized in high-power applications due to the reduction of both voltage stress across the power switches and voltage swing of the inductor [19]. These advantages allow for a significant reduction of the inductor size and semiconductor power losses. This chapter will introduce a multi-level non-inverting buck-boost (ML-NIBB) shown in Fig 4.1 and complementary controller for battery-powered application, which could be an alternative to the conventional non-inverting buck-boost converter. As shown in Fig 4.1, the power stage is a three-level buck in series connection with a three-level boost with a shared inductor between the two stages. In this topology, the utilization of flying capacitors allows for reduction of voltage swings of switching nodes on the both sides of the inductor, to a half of the values existing in the conventional NIBB. This allows a drastic reduction of the required inductance value. The ML-NIBB still has a smaller overall volume, even though two flying capacitors are used, since the capacitors have much higher power density than the inductor [28]. Also, power switches in ML-NIBB block a lower voltage in steady state operation, reducing switching losses and potentially allowing implementation with a semiconductor technology that has a better figure of merit (FOM). Furthermore, the smaller inductor in ML-NIBB has significantly smaller 
winding resistance, which allows further reduction of conduction losses. This means that even with more switches in the conduction path, both conduction and switching losses could potentially be reduced. Detailed comparison between conventional and multi-level converter is shown in [28].

In conventional NIBB, due to the nonideality of power switches, there is a practical limitation on maximum/minimum achievable duty ratio. Therefore, transition in the boundary of the step-up and the step-down has been a challenge for this topology. For the same reasons, this transition problem still exist in ML-NIBB. Furthermore, the duty ratio adjustments are appended on the duty ratio to balance the flying capacitors voltages. Therefore, a larger hysteretic band is needed for the transition between buck and boost modes in ML-NIBB. In this section, RS mode that is dicussed in the previous chapter will be extended to multilevel case, to accommodate the operation of ML-NIBB. MLRS mode has all the features mentioned in the previous chapter including achieving both step-up and step-down conversion ratio and regulation ability on inductor current. Since, with the utilization of flying capacitors, the voltage swing of the inductor is reduced, so as the inductor current ripple, allowing for even lower switching frequency. Therefore, ML-RS has higher efficiency improvement compared to the ripple-shaping mode of the conventional NIBB. Section 4.2 will present the extended version of RS mode called ML-RS, which is used as a transition mode between step-up and step-down mode in MLNIBB. Section 4.3 will show the comparison between conventional NIBB and ML-NIBB in terms of the volume and power processing efficiency. Then practical implementation and expereimental results will be shown comfirming improved performance of ML-NIBB and effectiveness of ML-RS based controller.

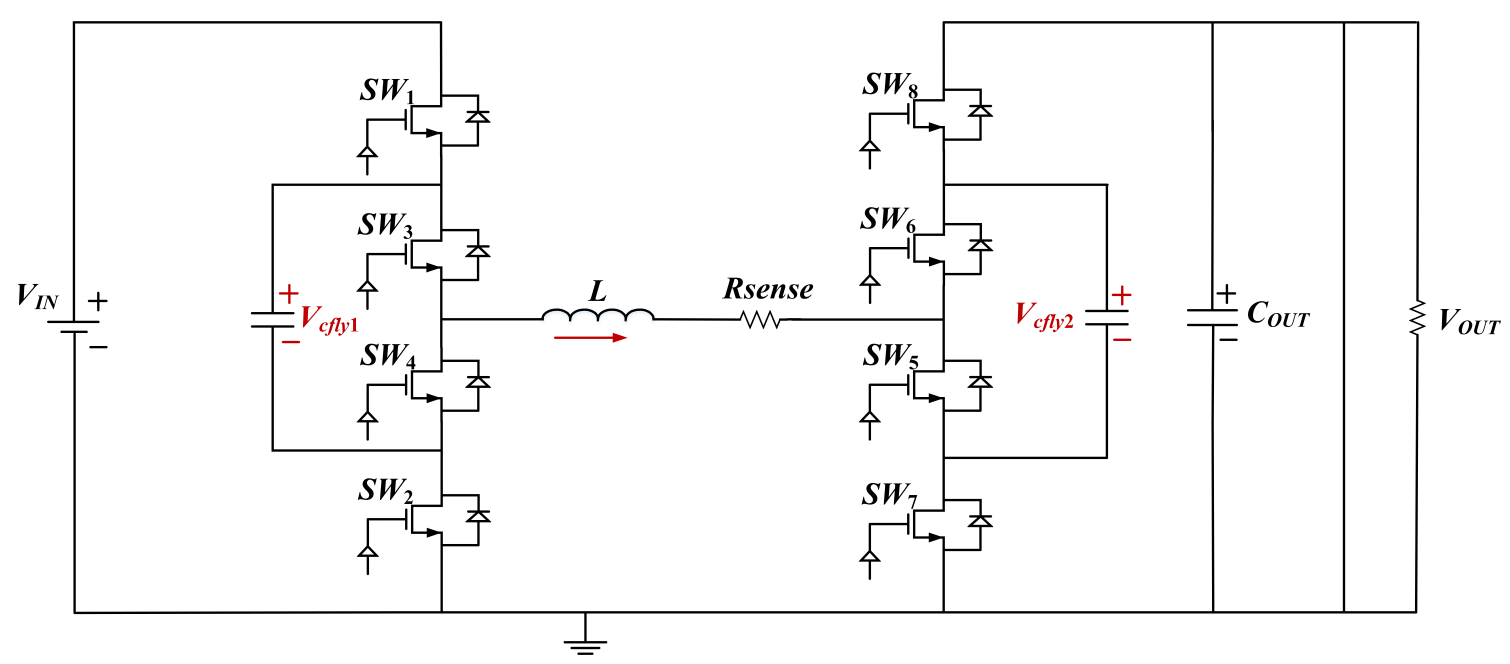

Figure 4.1: Multi-level Non-inverting buck-boost converter 


\subsection{Principle of Operation}

The ML-NIBB topology 4.1 has three modes of operation depending on the input voltage. When $V_{I N}$ is larger than $V_{\text {OUT }}$, it operates as a 3-level buck converter, where $S W_{5}$ and $S W_{7}$ are off. The converter switches among state1 to state4 depending on the different range of duty ratio. The steady-state operation of 3-level buck is extensively analyzed in [28]. When $V_{I N}$ is smaller than $V_{O U T}$, it operates as a 3-level boost converter, where $S W_{1}$ and $S W_{3}$ are on and the converter switches among state4 to state7. While the operation of 3-level boost is the dual version of the 3-level buck converter. The key waveforms including switching sequence, inductor current, capacitor current and switching nodes of 3-level buck and 3-level boost are shown in Fig 4.3 and 4.4. When $V_{I N}$ is close to $V_{\text {OUT }}$, the converter operates in ML RS mode.

The following figures are 7 possible subintervals of ML-NIBB converter operation including charge and discharge of input and output flying capacitor state1 Fig 4.2a,4.2b, 4.2e and 4.2f, direct connection between input and ouput voltage state4 Fig 4.2d. 


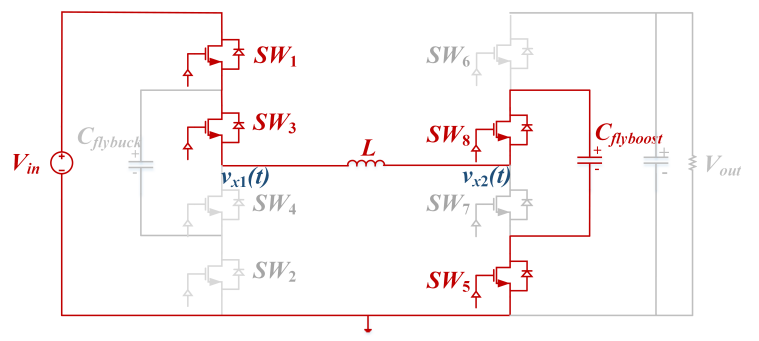

(a) State1

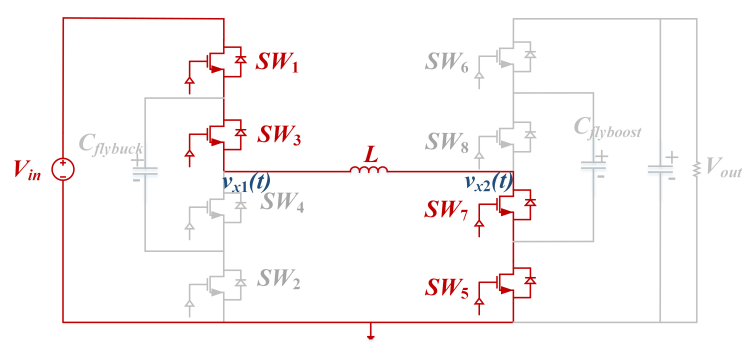

(c) State3

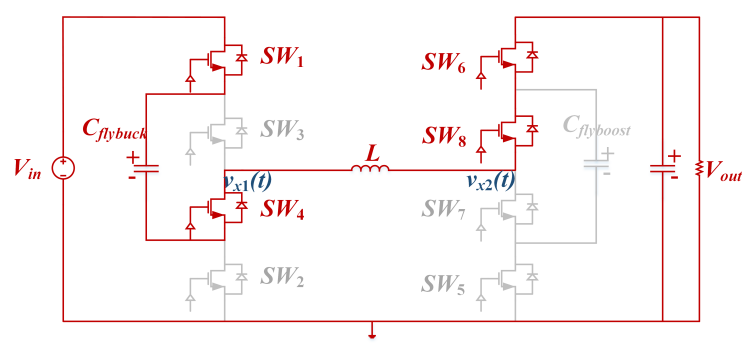

(e) State 5

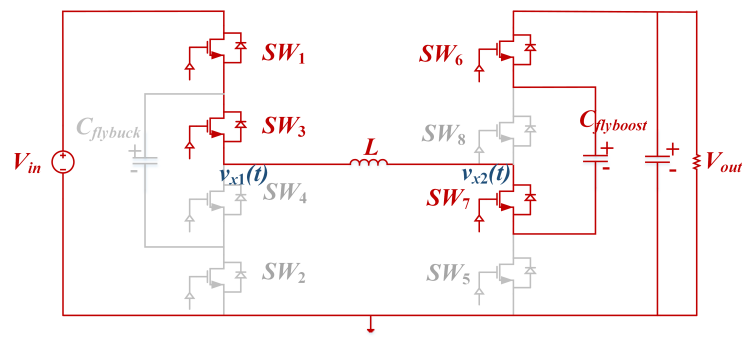

(b) State2

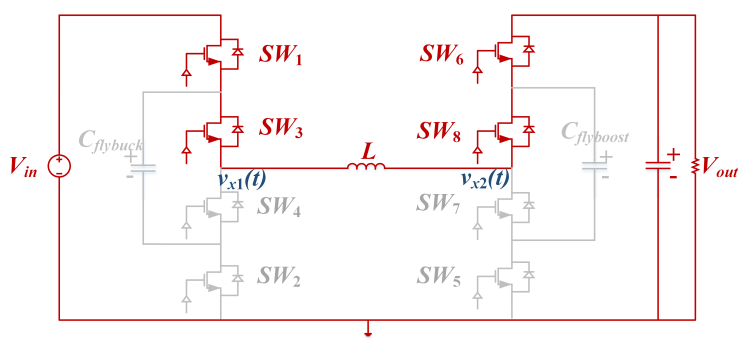

(d) State4

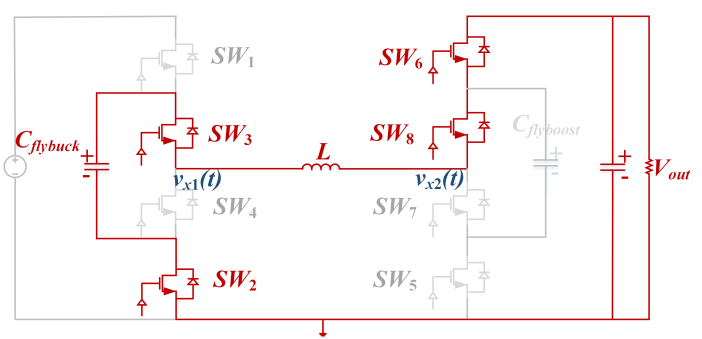

(f) State6

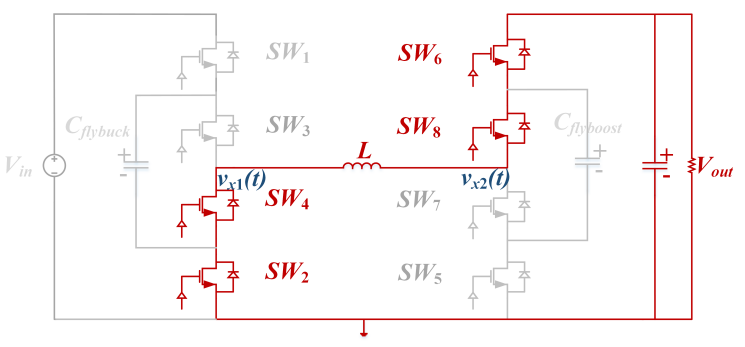

(g) State7

Figure 4.2: Subintervals of RS operation 

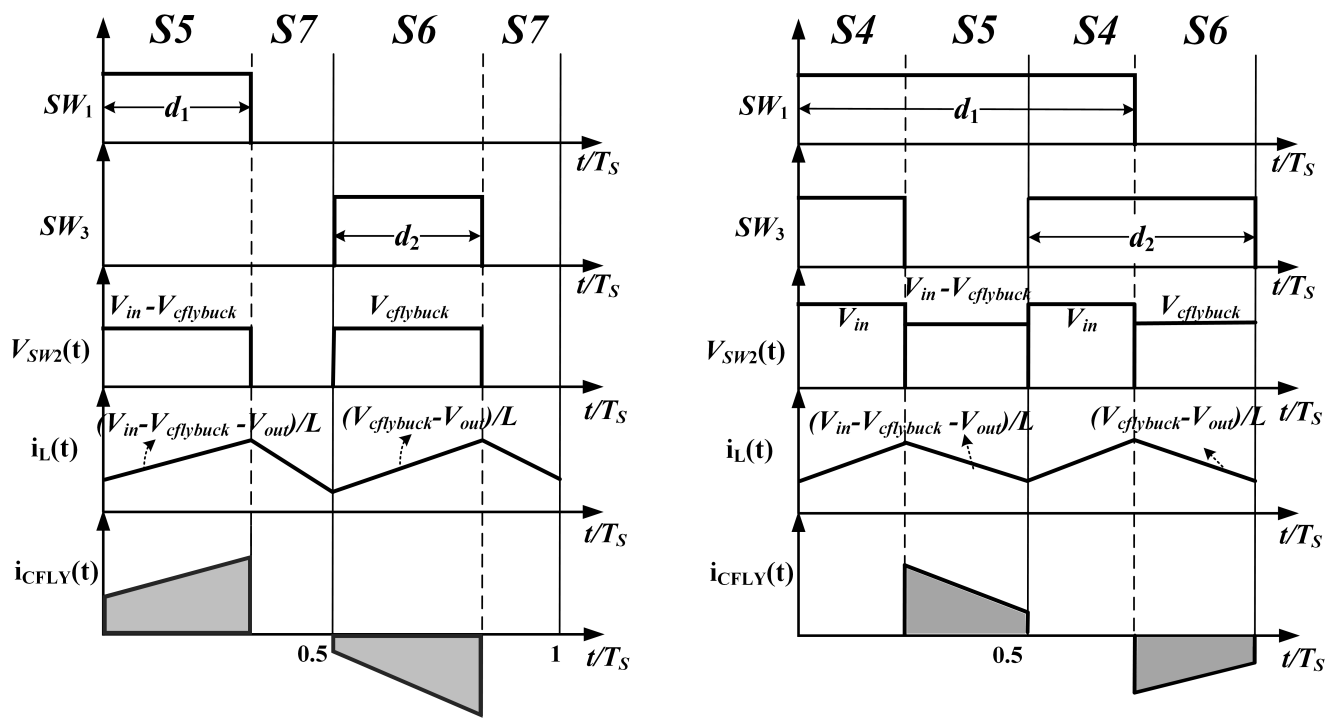

Figure 4.3: 3-level buck operation when duty ratio is smaller than 0.5 (left) and when duty ratio is larger than 0.5 (right)
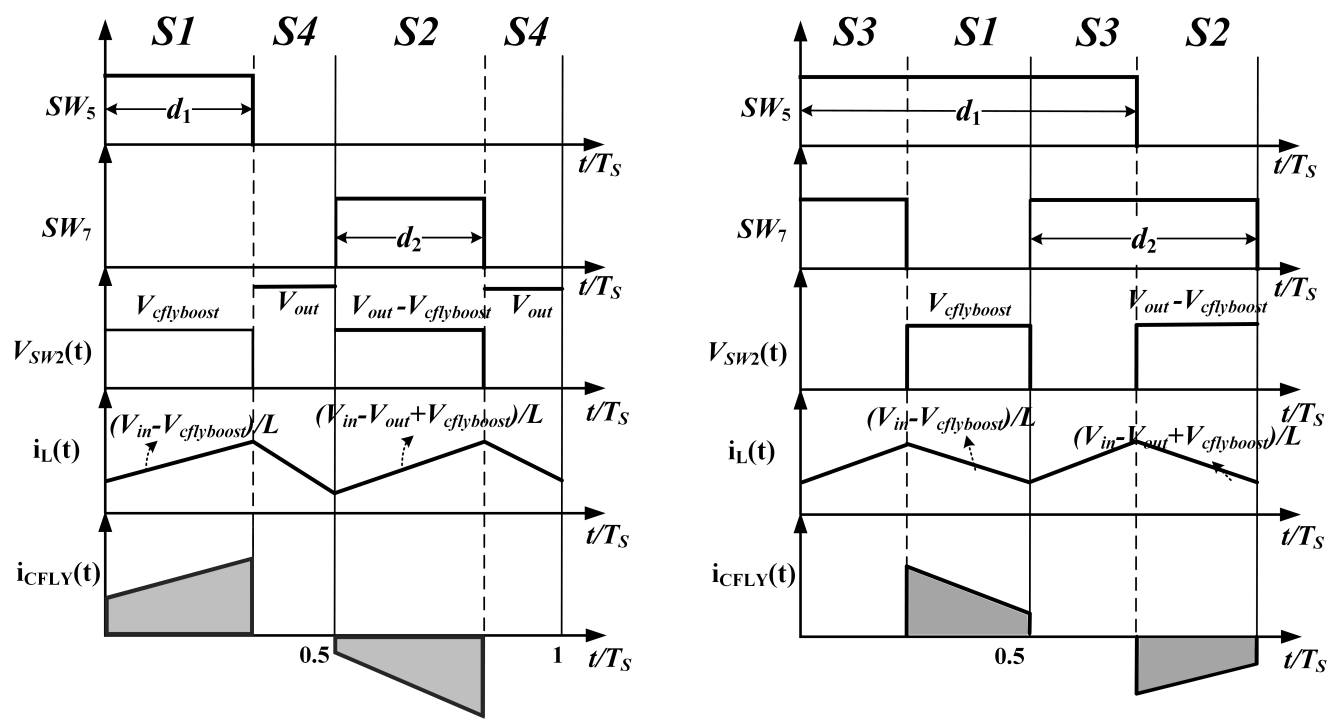

Figure 4.4: 3-level boost operation when duty ratio is smaller than 0.5 (left) and when duty ratio is larger than 0.5 (right)

\subsubsection{ML-RS Mode}

The key waveforms of the ML-RS are given in Fig 4.5. The waveforms show gating sequence, the input and output switching node voltages, currents of the input and output flying capacitors and the inductor currents. It can be seen that, there are 8 subintervals 
in one switching cycle. Under the assumption that the flying capacitors are balanced. The input switching node voltage switches between $V_{I N}$ and $V_{I N} / 2$. The duty ratio of $S W_{1}$ and $S W_{3}$ are greater than 0.5 . While the output switching node voltage switches between $V_{\text {OUT }}$ and $V_{\text {OUT }} / 2$. The duty ratio of $S W_{5}$ and $S W_{7}$ is smaller than 0.5 . It can be seen that the voltage swing on both side of the inductor reduced to half of the input and output voltage, which reduces blocking voltage of the switches and inductor current ripple.

The full input or output voltage swing on inductor like state 3 Fig $4.2 \mathrm{c}$ and state 7 Fig $4.2 \mathrm{~g}$ are avoided. In addition, no state has both flying capacitors on the conduction path at the same time. Even though, it could provide smaller voltage across the inductor compared to direct connection shown in the state4 Fig $4.2 \mathrm{~d}$, the duty ratio of $S W_{5}$ and $S W_{7}$ will increase. This will increase the average inductor current, i.e. RMS current. Therefore, the switching sequence shown in Fig 4.5 is the optimal way to reshape the inductor current to reduce the RMS value. 


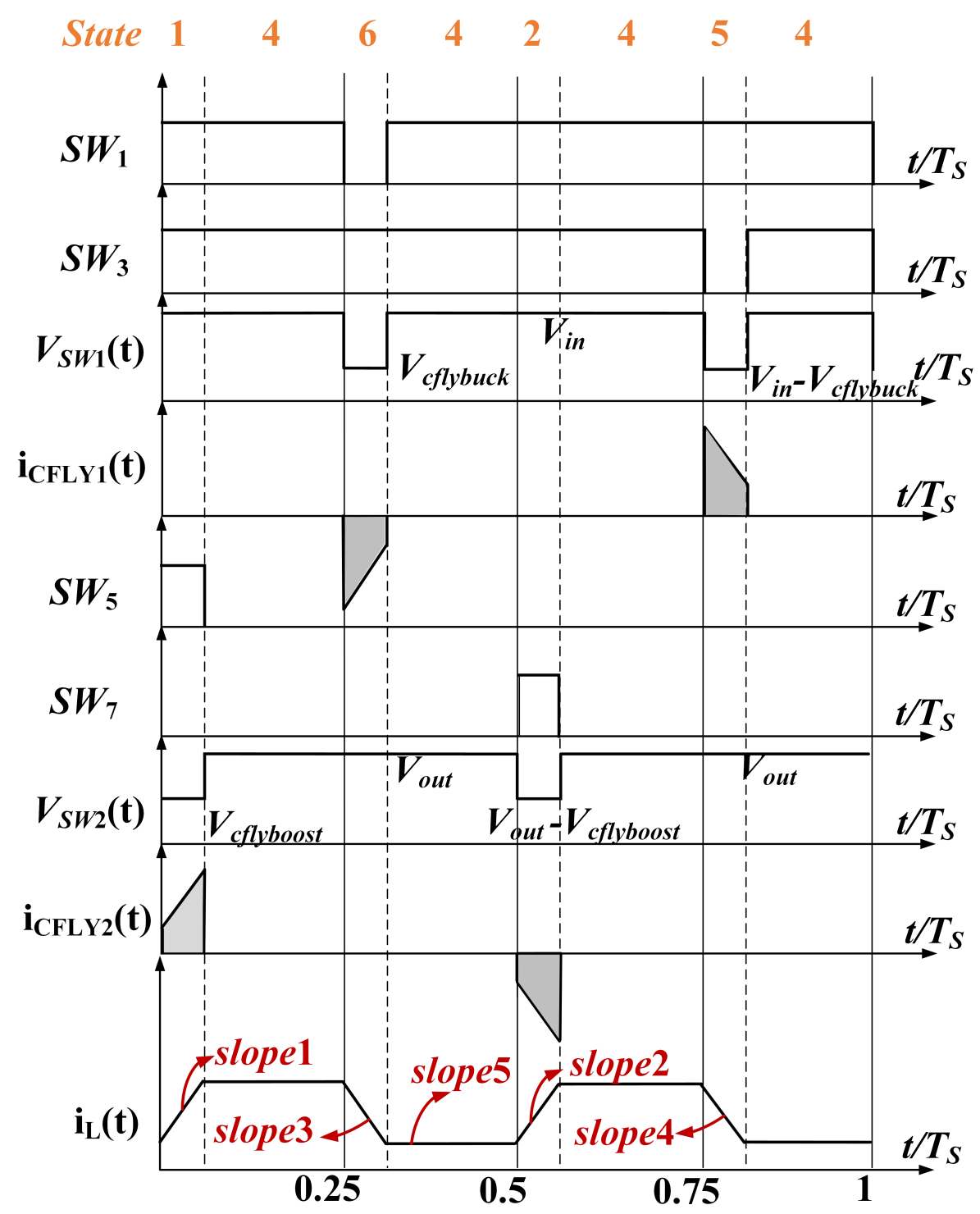

Figure 4.5: Key waveforms of multi-level ripple-shaping including switching sequence, switching node voltages, corresponding inductor current waveform

According to inductor volt-second balance equation [17], conversion ratio of 3-level buck, 3-level boost and ML-RS are shown in the following:

$$
\begin{gathered}
M\left(D_{1}, D_{2}\right)_{R S}=\frac{D_{1}}{1-D_{2}} \\
M\left(D_{1}\right)_{b u c k}=D_{1}, \quad \text { and }
\end{gathered}
$$




$$
M\left(D_{2}\right)_{b o o s t}=\frac{1}{1-D_{2}}
$$

According to capacitor-charge balance [17], the inductor current equation is shown in the following:

$$
\begin{gathered}
I_{L(\text { ave }) R S}=I_{\text {Load }} \times \frac{1}{1-\frac{\text { ton }_{S W_{3}}}{T_{S}}}, \\
I_{L(\text { ave }) \text { buck }}=I_{\text {Load }} \text {, and } \\
I_{L(\text { ave }) \text { boost }}=I_{\text {Load }} \times\left(\frac{1}{1-D_{2}}\right)
\end{gathered}
$$

$D_{1}$ is the ratio between the turn on time of $S W_{1,3}$ and period $T_{S}, D_{2}$ is the ratio between turn on time of $S W_{5,7}$ and period $T_{S}$. Equation show ML-NIBB has the same conversion ratio as conventional NIBB. And ML-RS preserves the ability of step-up and step-down conversion ratio and regulating the inductor current to be almost load current.

\subsection{Inductor volume reduction and efficiency improve- ments}

\subsubsection{Inductor volume reduction}

The main objective of this section is to compare the conventional NIBB with ML-NIBB in terms of the inductor volume and power processing efficiency. Comparison is under the assumption of same switching node frequency and the same inductor current ripple [28]. There are two cases that are taken into account. One is with various duty ratio and same inductor current ripple design requirement, maximum inductance in both converter can be compared. The other case is comparing the inductance at fix conversion ratio for a given ripple requirement. In the buck case, peak current equals to load current plus inductor current ripple, under the assumption of same inductor current ripple, the peak current is the same among all conversion ratio. While in the boost case, peak current changes with duty ratio. As mentioned before, the volume of inductor depends on the energy storage capacity. According to the equation, when the peak current is the same, the volume of the inductor is proportional to the value of inductance. Therefore, both cases can be used to observe the reduction of inductor volume based on the inductance in buck. However, in step up, under fixed conversion ratio, the volume reduction is 
proportional to the inductance reduction, but with various conversion ratio the volume reduction is a nonlinear function of both inductance and peak current. The inductance for both topologies under different operation regions are shown in the following.

Based on volt-second balance analysis [17], the inductance for a conventional NIBB in buck, boost, ripple-shaping step down (RS SD) and ripple-shaping step up (RS SU) mode are shown in the following expressions:

(i) Buck

$$
L=\frac{V_{I N}-V_{\text {OUT }}}{\Delta i_{L p p} \times f_{\text {ripple }}} \times D
$$

(ii) Boost

$$
L=\frac{V_{\text {OUT }}-V_{I N}}{\Delta i_{\text {Lpp }} \times f_{\text {ripple }}} \times D^{\prime}
$$

(iii) RS step down

$$
L=\frac{\left(M(D)-M^{2}(D) \times\left(1-D_{\text {min }}\right)\right.}{\Delta i_{\text {Lppripplers }}} \times V_{I N},\left\{M(D)=\frac{D}{1-D_{\min }}\right\}
$$

(iv) RS step up

$$
L=\frac{M(D)-1+D_{\min }}{\Delta i_{\text {Lpp }} \times f_{\text {ripplers }} \times M(D)} \times V_{I N},\left\{M(D)=\frac{D_{\max }}{1-D}\right\}
$$

The inductance for a ML-NIBB in 3-level buck can be calculated from the following expressions:

(i) For $0<\mathrm{D}<0.5$ :

$$
L=\frac{\frac{1}{2} \cdot V_{I N}-V_{\text {OUT }}}{\Delta i_{\text {Lpp }} \times 2 \times f_{\text {ripple }}} \times D
$$

(ii) For $0.5<\mathrm{D}<1$ :

$$
L=\frac{\frac{1}{2} \times V_{I N}-V_{\text {OUT }}}{\Delta i_{\text {Lpp }} \times 2 \times f_{\text {ripple }}} \times(D-1)
$$


The inductance for a ML-NIBB in 3-level boost can be calculated from the following expressions:

(i) For $0<\mathrm{D}<0.5$ :

$$
L=\frac{V_{I N}-\frac{1}{2} \times V_{\text {OUT }}}{\Delta i_{\text {Lpp }} \times 2 \times f_{\text {ripple }}} \times D
$$

(ii) For $0.5<\mathrm{D}<1$ :

$$
L=\frac{V_{I N}-\frac{1}{2} \times V_{\text {OUT }}}{\Delta i_{\text {Lpp }} \times 2 \times f_{\text {ripple }}} \times(D-1)
$$

The inductance for a ML-NIBB in ML-RS can be calculated from the following expressions:

(i) ML-RS step down

$$
L=\frac{V_{I N}}{\Delta i_{\text {Lpp }} \times 2 \times f_{\text {ripplers }}} \times-M^{2}(D) \times\left(1-D_{\text {min }}\right)+M(D) \times\left(\frac{3}{2}-\frac{1}{2} \times D_{\text {min }}\right)-\frac{1}{2}
$$

where $M(D)=\frac{D}{1-D_{\min }}$

(ii) ML-RS step up

$$
L=\frac{V_{I N}}{\Delta i_{\text {Lpp }} \times 2 \times f_{\text {ripplers }}} \times \frac{-\frac{1}{2} \times M^{2}(D)+M(D) \cdot\left(\frac{3}{2}-\frac{1}{2} \times D_{\text {min }}\right)-1+M_{\min }}{M(D)},
$$

where $M(D)=\frac{D_{\max }}{1-D}$

and $\Delta i_{L p p}$ is the peak to peak inductor current, $f_{\text {ripple }}$ is the ripple frequency in buck or boost mode, $f_{\text {ripplers }}$ is the ripple frequency in ripple-shaping mode. $D$ is the duty ratio, $M(D)$ is the conversion ratio of corresponding operation mode, the input and output voltages are $V_{I N}$ and $V_{O U T}$ respectively.

The following figure Fig 4.6 shows the normalized inductance value comparison in conventional NIBB and ML-NIBB converter with respect to largest inductance value in deep boost assumed the largest conversion ratio is 2 . For the conversion ratio smaller than 1 , worst, i.e. largest inductance, occurs when the duty ratio equals to 0.25 or 0.75 in 
the 3-level buck. While for 2-level, i.e. conventional buck, worst case inductance occurs at duty ratio equal to 0.5 . It can be seen that, the worst inductance is half in multilevel compared to conventional buck. For certain duty ratio and inductor current ripple, for example, when the duty ratio is 0.4 , the 3 -level buck only need $1 / 3$ of the inductor volume compared to 2-level buck.

In the case where conversion ratio is larger than 1 , for the certain conversion ratio that peak current is the same for both converter under the assumption that same inductor current ripple are obtained, the volume could be reduced proportionally to the reduction of inductance. For example, when conversion ratio equals to 1.4, 3-level boost needs only $56 \%$ of inductance in 2-level boost. For the various duty ratio, the worst-case inductance reduction depends on the conversion ratio, 3-level boost benefits more in high step down range. Worst inductance shown in the figure where the maximum conversion ratio is 2 , is $34 \%$ of the inductance needed in 2-level boost. In the whole range operation of MLNIBB, the overall reduction of inductance depends on the highest conversion ratio. Under consumption here, i.e. maximum conversion ratio is 2, worst inductance in ML-NIBB is only $34 \%$ of that in conventional.

In the case of RS mode, both RS and ML-RS have much lower switching frequency, e.g. $1 / 5$ of the buck/boost mode. The inductor current ripple increases at the transition point and decreases as $V_{I N}$ approaching to $V_{O U T}$. The inductor current ripple reaches the minimum value as buck or boost mode. It should be noted that the inductor selection is not based on the maximum ripple in RS mode, which depends on the switching frequency. For choosing inductance, based on the worst situation in boost, as long as the peak current in RS mode does not exceed the peak current in boost mode, no larger inductor is needed. 


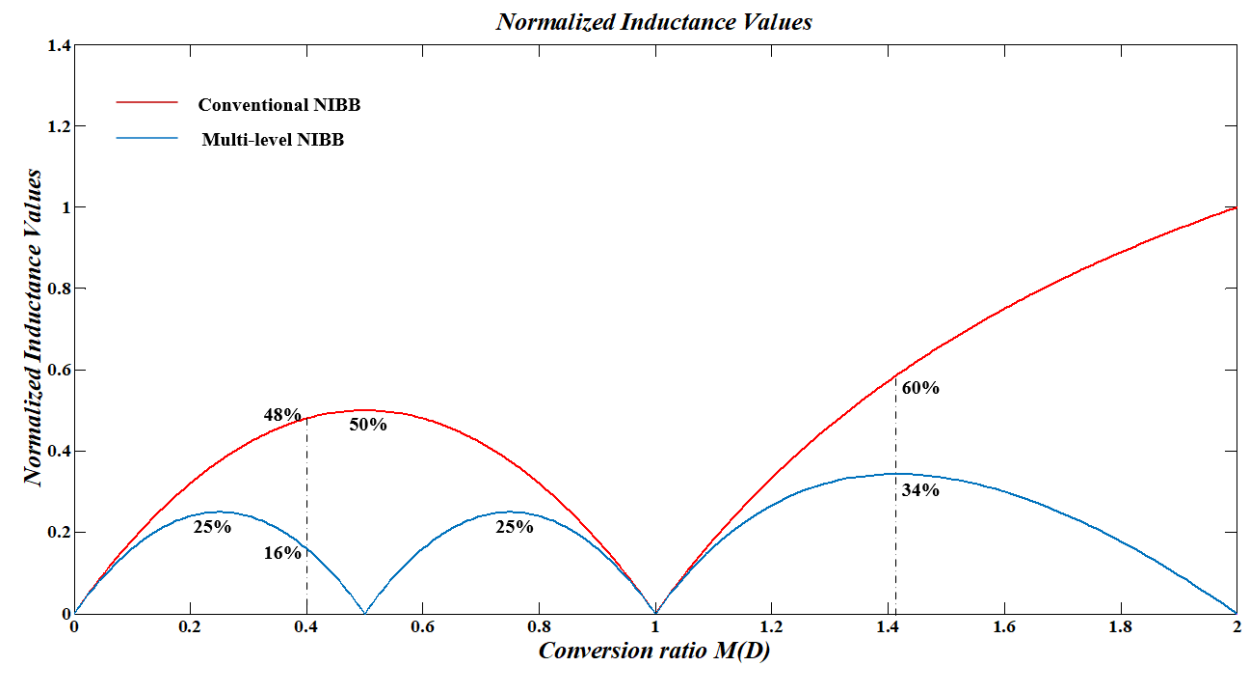

(a) Normalized inductor values in buck, boost mode with respect to worst inductance in boost

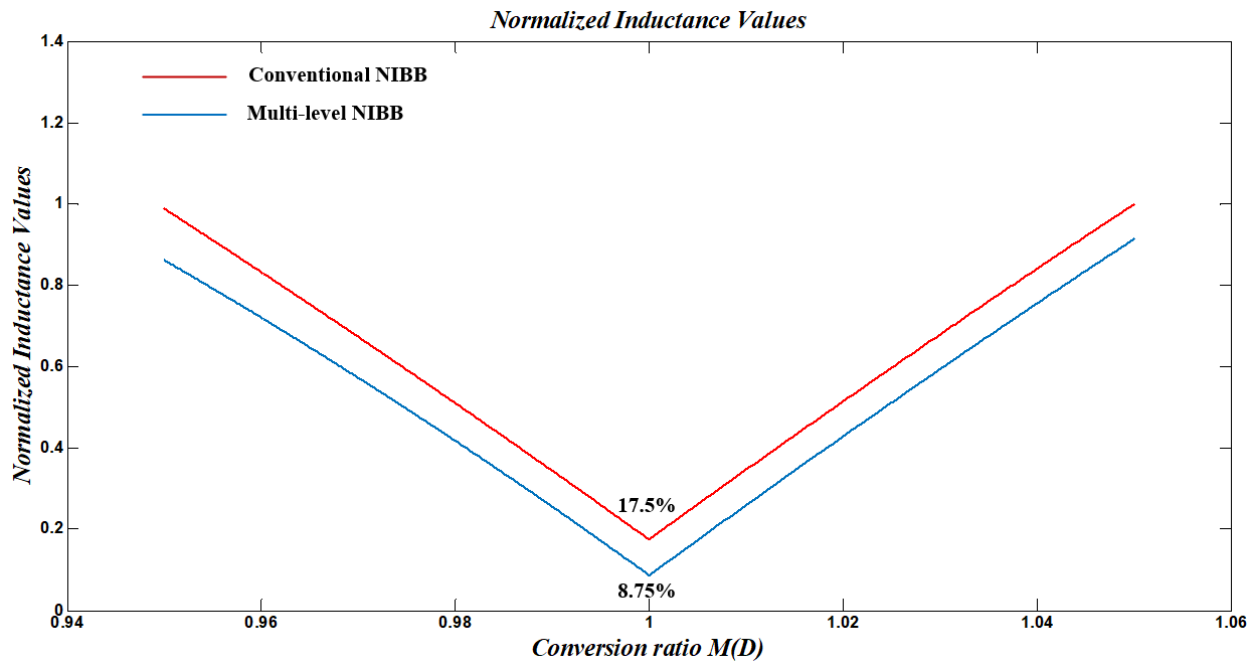

(b) Normalized inductor values in RS mode

Figure 4.6: Normalized inductor values in NIBB and ML-NIBB with respect to worst inductance in transition region

\subsubsection{Efficiency improvement}

In multi-level NIBB converter, twice the number of switches are conducting in each mode. However, switching node voltage reduced to half of conventional NIBB, which allows the power switches have a half of the blocking voltage. Therefore, switches with better FOM can be implemented in the target application. According to the equation, $R_{o n}=\alpha V_{b k}^{2}$, 
halved blocking voltage allows 4 times smaller on resistance. Therefore, even with the same silicon area for twice the switches, the resistance of the conduction path could be the same. In terms of switching losses, ML-NIBB has smaller blocking voltage and less switching activities if same inductor current ripple, i.e. the same ripple amplitude and frequency, is required. Switching losses of each switch consists of two parts. One is associated to the charging and discharging of the output capacitor and the other to hard switching losses which relate to the blocking voltage. Under the same inductor ripple amplitude, multi-level allows much lower switching frequency. For example, when the same worst-case ripple and half inductance is required, switching frequency could be reduced to half. Smaller $V_{v k}$ contributes losses reduction on output capacitors and hard switching losses. The switching loss of each switch is shown in the following [17]:

$$
P_{S W}=0.5 \times f_{s} \times\left(V_{b k} \times I_{L} \times\left(t_{r}+t_{f}\right)+V_{b k}^{2} \times C_{o s s}\right)
$$

where $f_{s}$ is the switching frequency, $V_{b k}$ is switch blocking voltage, $t_{r}$ is the rising time and $t_{f}$ is the falling time, $C_{\text {oss }}$ is the output capacitance.

\subsection{Practical Implementation}

The practical implementation of the introduced ML-NIBB with ML-RS logic control is shown in Fig 4.7. The FPGA based digital controller is a modification of a conventional average CPM controller. In this case, the controller also contains Mode Selection Logic that decides the preferable mode of operation based on the input voltage information with hysteretic band implemented to avoid mode toggling. Accordingly, ML-RS logic block is bypassed when the converter operates as a 3-level buck or a 3-level boost. Or in RS mode, it fixes either buck or boost side of switches duty ratio to the maximum or the minimum value. Gating signals have 90-degree phase shift between $S W_{1,3}$ and $S W_{5,7}$ to shape inductor current into the trapezoidal waveform. In addition, duty-cycle offsetting mechanism block is appended to provide a well-balanced flying capacitor voltage. Besides, transition techniques including frequency scaling and compensator reset that are used in conventional RS mode controller (described in section 3.4) are also implemented here to obtain stable mode transitions in terms of inductor current and output voltage variations. 


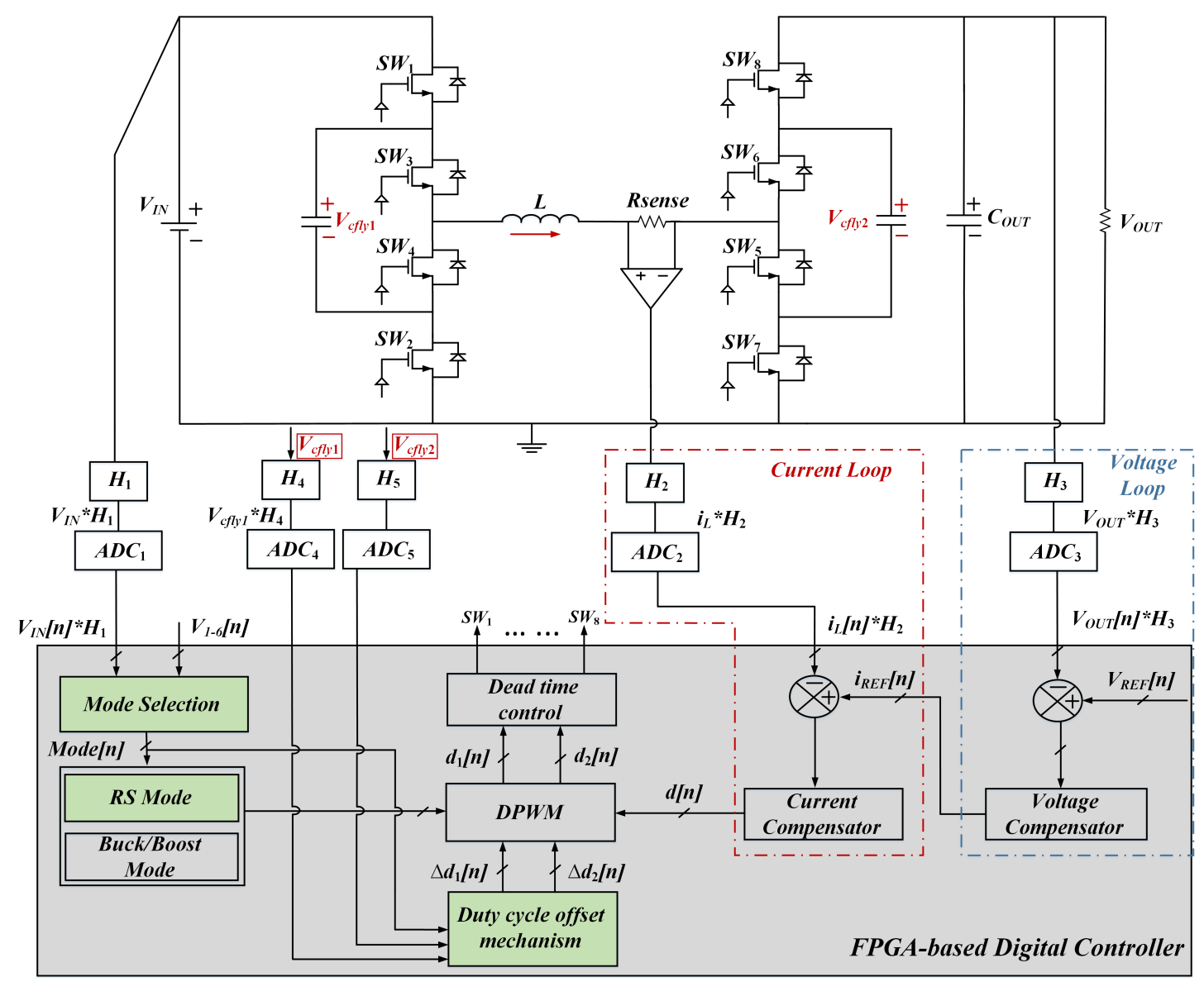

Figure 4.7: Multi-level Non-inverting buck-boost converter regulated with low-frequency multi-level ripple-shaping controller,implemented through digital average current mode controller

\subsubsection{Discrete prototypes parameters}

Table 4.1: Converter parameters for RS controller verification

\begin{tabular}{|c|c|}
\hline Parameters & Value \\
\hline$V_{\text {IN }}$ & $1.8 \mathrm{~V}-5.5 \mathrm{~V}$ \\
\hline$V_{\text {OUT }}$ & $3.3 \mathrm{~V}$ \\
\hline$I_{\text {OUT }}$ & $0.5 \mathrm{~A}-3 \mathrm{~A}$ \\
\hline$f_{\text {Sbuck } / \text { boost }}$ & $500 \mathrm{kHz}$ \\
\hline$f_{\text {SRS }}$ & $100 \mathrm{kHz}$ \\
\hline$L$ & $1.1 \mu \mathrm{H}$ \\
\hline$C_{\text {OUT }}$ & $45 \mu \mathrm{F}$ \\
\hline
\end{tabular}


Table 4.2: Converter Parameters for efficiency comparison

\begin{tabular}{|c|c|c|}
\hline Parameters & Conventional & Multi-level \\
\hline Inductor Value & $2.2 \mu \mathrm{H}[29]$ & $1 \mu \mathrm{H}[30]$ \\
\hline Inductor current rating & $4.7 \mathrm{~A}$ & $5 \mathrm{~A}$ \\
\hline$R_{L}$ & $29 \mathrm{~m} \Omega$ & $20 \mathrm{~m} \Omega$ \\
\hline Inductor Volume & $4.1^{*} 4.1^{*} 2.1 \mathrm{~mm}^{3}$ & $3.65^{*} 3^{*} 2 \mathrm{~mm}^{3}$ \\
\hline Flying cap & - & $2 \times 5 \mu F$ \\
\hline Flying cap Volume & - & $1.6^{*} 0.8 * 0.9 \mathrm{~mm}^{3}$ \\
\hline$f_{\text {sw }}$ & $1 \mathrm{MHz} / 200 \mathrm{kHz}$ & $500 \mathrm{kHz} / 100 \mathrm{kHz}$ \\
\hline$f_{i L}$ & $1 \mathrm{MHz} / 200 \mathrm{kHz}$ & $1 \mathrm{MHz} / 200 \mathrm{kHz}$ \\
\hline Switches & $\mathrm{IRF} 7478$ & $\mathrm{IRF} 6342$ \\
\hline$R_{\text {on }}$ & $20 \mathrm{~m} \Omega$ & $11 \mathrm{~m} \Omega$ \\
\hline$C_{i s s}$ & $1740 \mathrm{pF}$ & $1025 \mathrm{pF}$ \\
\hline$C_{\text {oss }}$ & $300 \mathrm{pF}$ & $91 \mathrm{pF}$ \\
\hline$Q_{g}$ & $21 \mathrm{nC}$ & $11 \mathrm{nC}$ \\
\hline Passive Components Volume saving & - & $11.1 \mathrm{~mm}{ }^{3}$ \\
\hline
\end{tabular}

Discrete prototypes of both multi-level and conventional non-inverting buck-boost have been built to compare the performance of two solutions. Digital control is implemented on FPGA based system. The input voltage range is from $4 \mathrm{~V}$ to $10 \mathrm{~V}$, output voltage range is $4 \mathrm{~V}$ to $10 \mathrm{~V}$ and output current is no larger than $2 \mathrm{~A}$, which covers deep buck, shallow buck, deep boost, shallow boost, and ML-RS operation. The inductor selection is based on worst case inductor current ripple in buck/3-level buck and boost/3-level boost. The comparison is performed based on the assumption that both topologies result in the same inductor current ripple frequency and worst maximum ripple amplitude in 3-level buck [28]. Transistors in ML-NIBB are selected such that switches have a half voltage rating compare to conventional NIBB. Thus, allowing their implementation with components having better figure of merit (FOM). The FOM has been improved 3 times, where the FOM, is taken as a product of the Ron resistance of the transistor and the equivalent capacitances of the transistor [31]. The converters for the target application that the input voltage range is $1.8 \mathrm{~V}$ to $5 \mathrm{~V}$ and output voltage is $3.3 \mathrm{~V}$, are usually integrated on an IC. Due to the limited selection of discrete components, there is no proper switch rating in that range with 3 times FOM improvement available. However, for IC implementation, this is not an issue. In this thesis, discrete components prototype is build to verify the idea. Therefore, the discrete prototype uses IRF 7478 switches with 
a voltage rating of $60 \mathrm{~V}$ for the conventional architecture and IRF 6342 switches with a voltage rating of $30 \mathrm{~V}$ for the multi-level architecture. These switches are suitable for building a large-scale prototype, to replicate the FOM improvements shown in the table. The total volume of inductor, which is by far the largest reactive component of the conventional converter, has been reduced by more than 2.3 times at the expense of two flying capacitors which total volume is less than a $3 \%$ of the inductor volume in the conventional converter. Flying capacitors are selected that voltage ripple amplitude is about $10 \%$ magnitude lower than the input/output voltage [28]. The inductor current ripple frequency is chosen to be $1 \mathrm{MHz}$ in buck/boost mode and $200 \mathrm{KHz}$ in RS mode. Therefore, with the utilization of flying capacitors, ML-NIBB prototype switches at 500 $\mathrm{KHz}$ in buck/boost mode and $100 \mathrm{KHz}$ in RS mode while the conventional NIBB switches at $1 \mathrm{MHz}$ and $200 \mathrm{KHz}$ respectively. The efficiency comparison results showing in the following proves ML-NIBB has higher efficiency. Detailed information about the discrete components used in the prototypes are shown in Table 4.2. The effectiveness of ML-RS controller is verified on the same discrete prototype.

\subsubsection{Efficiency Comparison}

The following figures are efficiency comparison between ML-NIBB and conventional NIBB in $\mathrm{RS}$

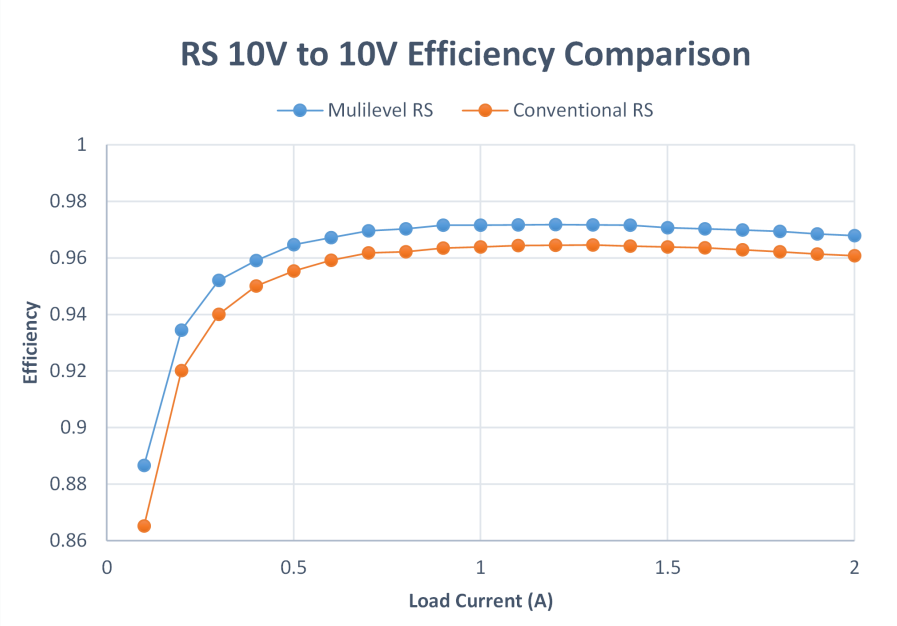

Figure 4.8: Efficiency comparison between ML-RS and conventional RS 


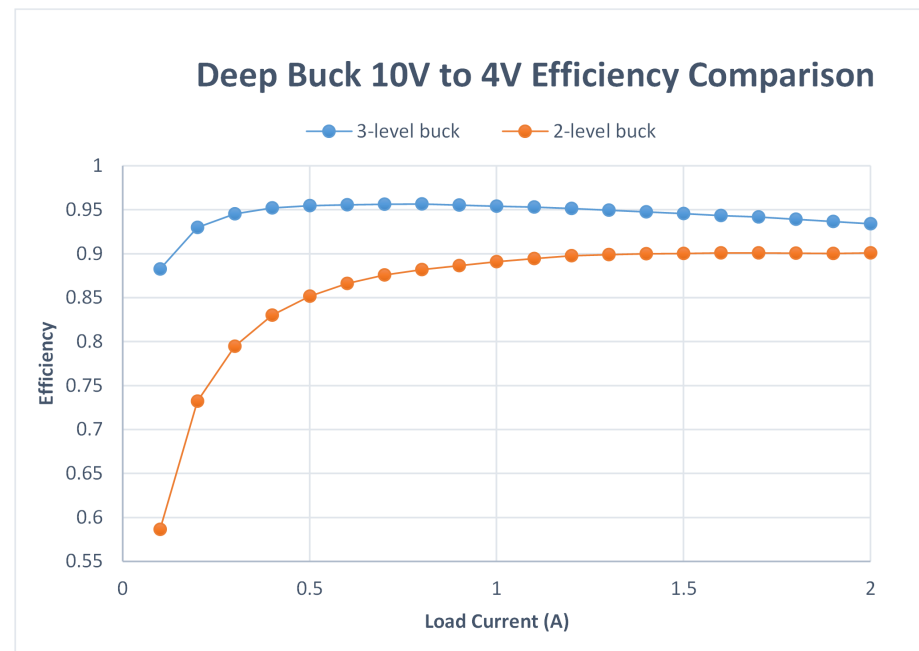

Figure 4.9: Efficiency comparison between 3-level buck and 2-level buck in duty ratio smaller than 0.5

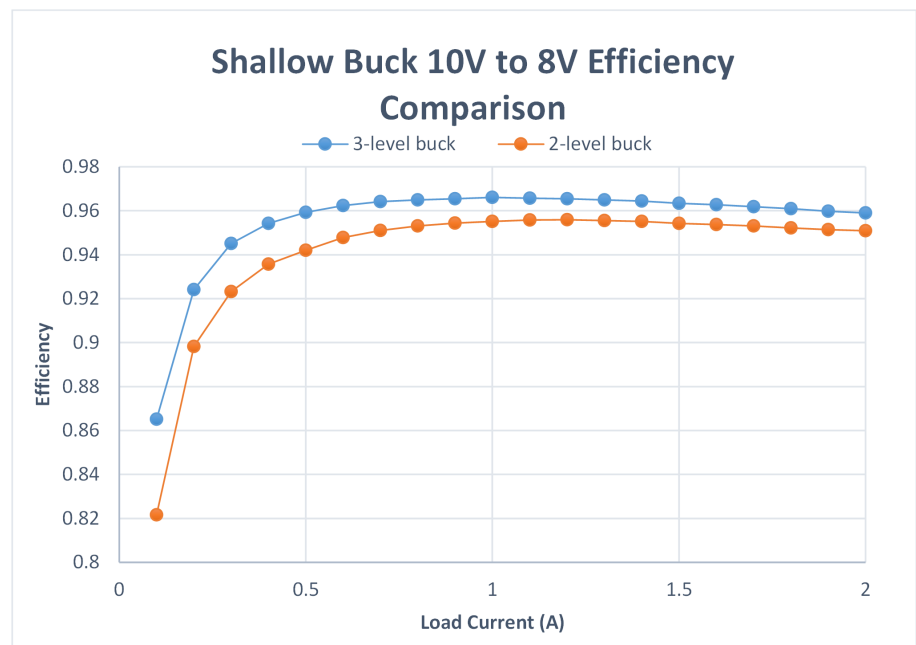

Figure 4.10: Efficiency comparison between 3-level buck and 2-level buck in duty ratio larger than 0.5 


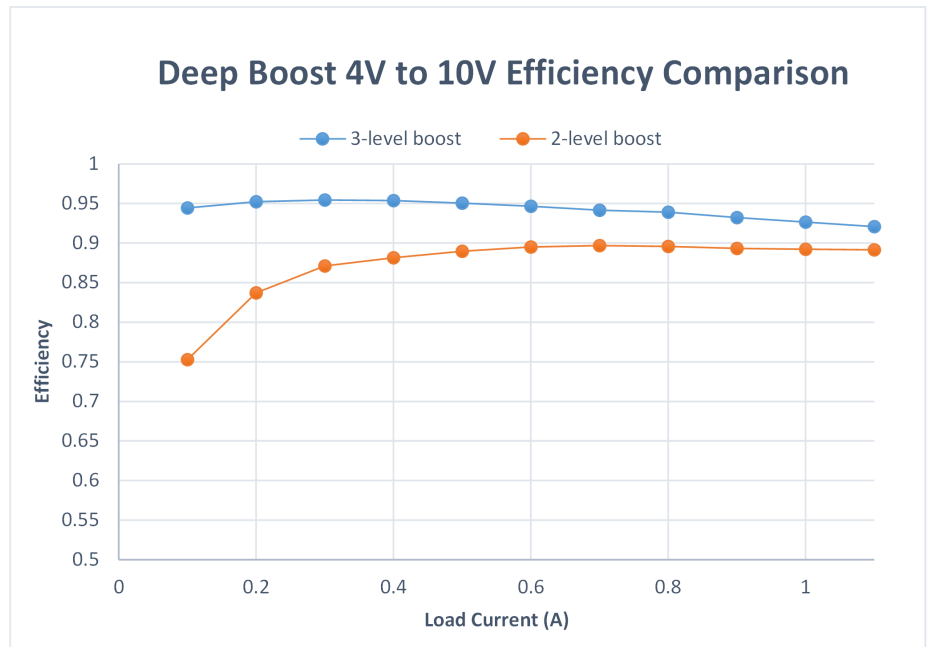

Figure 4.11: Efficiency comparison between 3-level boost and 2-level boost in duty ratio larger than 0.5

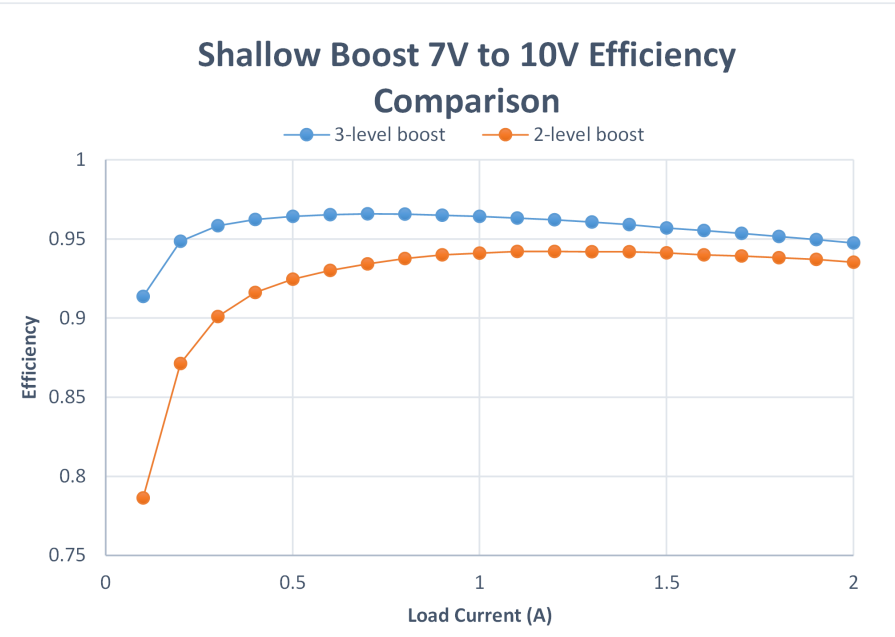

Figure 4.12: Efficiency comparison between 3-level boost and 2-level boost in duty ratio smaller than 0.5

The efficiency comparison shown in Fig. 17 to Fig. 21 are measured results, which demonstrate up to $20 \%$ power processing efficiency improvement in light load and $5 \%$ in medium load and around 3\% in heavy load of the ML-NIBB the converter through the entire range of operation. 


\subsubsection{ML-NIBB Operation}

A modified digital average CPM controller is built [16]. With the duty-ratio offsetting mechanism, steady-state operation of each mode are shown in Fig 4.13, which verify the effectiveness of ML-RS controller operating in the dead zone. Additionally, small inductor current ripple can be observed even with 5 times smaller operating frequency.

Fig 4.13 represent the respective modes of operation including 3-level buck, 3-level boost, ML-RS step down and ML-RS step up. Fig 4.13a and 4.13b show the 3-level buck and 3-level boost mode operation. Fig 4.13c and 4.13e show the RS mode near the transition point where the discrepancy between input and output voltage is ineligible. The switching frequency in ML-RS mode is $1 / 5$ of 3-level buck/boost mode. And there are eight subintervals in one switching cycle. Fig 4.13d and 4.13f present the case where voltage across the inductor is almost 0 . The current ripple is about the same with conventional NIBB, which is about $500 \mathrm{~mA}$ with halved inductance and switching frequency. However, from the efficiency point of view larger advantages are gained. Furthermore, the effectiveness of duty raito offsetting mechanism is proved. By looking at the switching node voltages and inductor current, flying capacitor voltages are well balanced. 


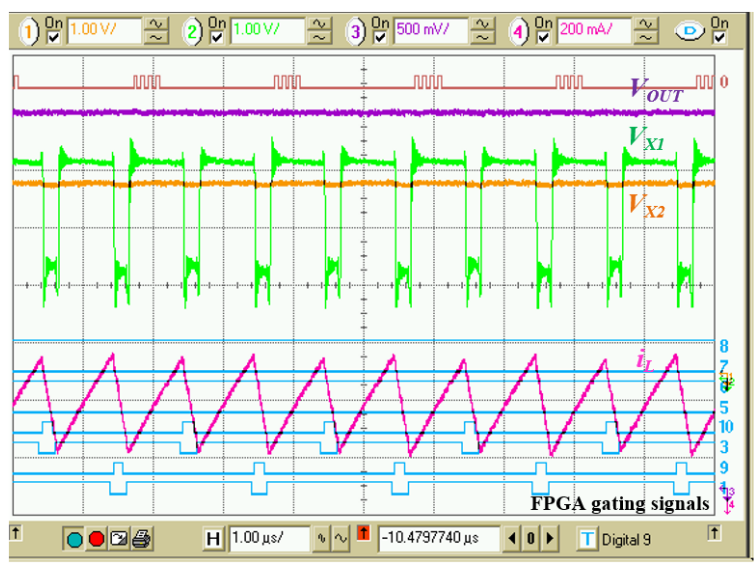

(a) Buck Mode

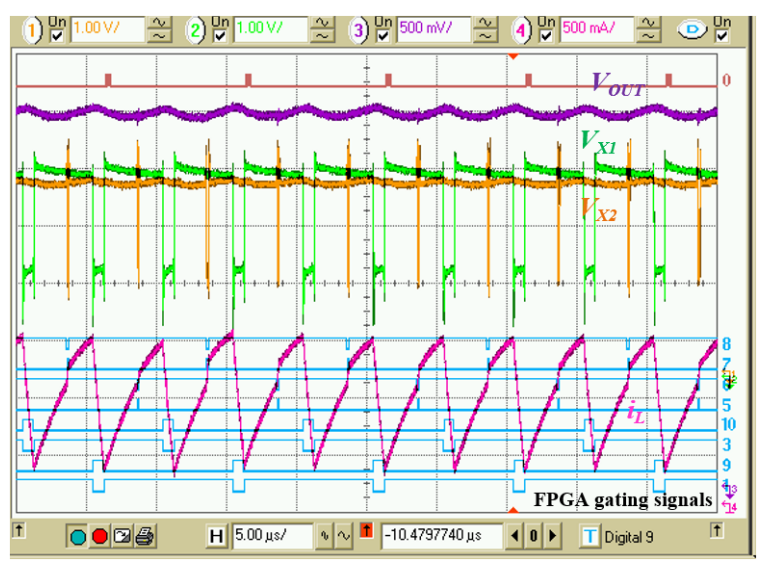

(c) RS step-down mode

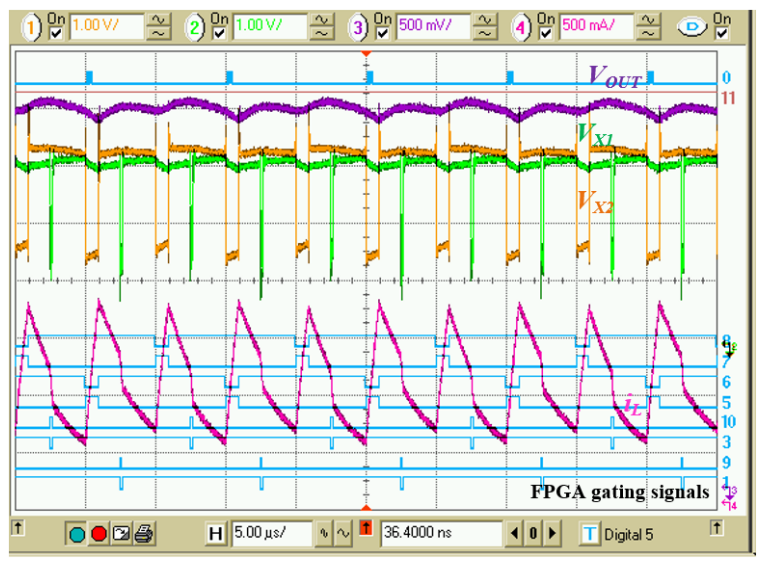

(e) RS step-up mode

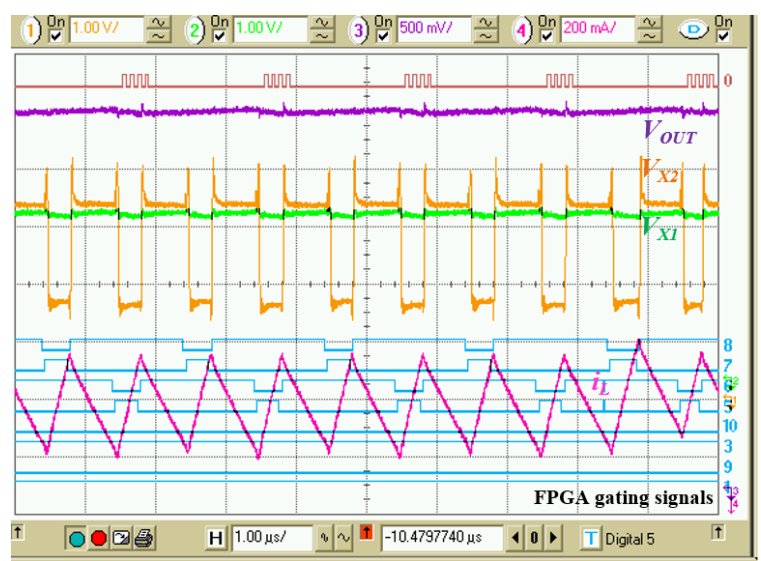

(b) Boost Mode

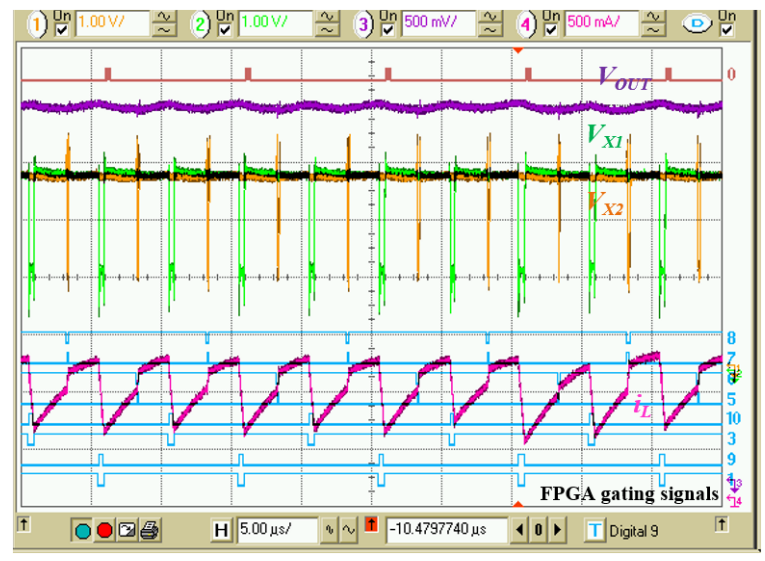

(d) RS step-down mode

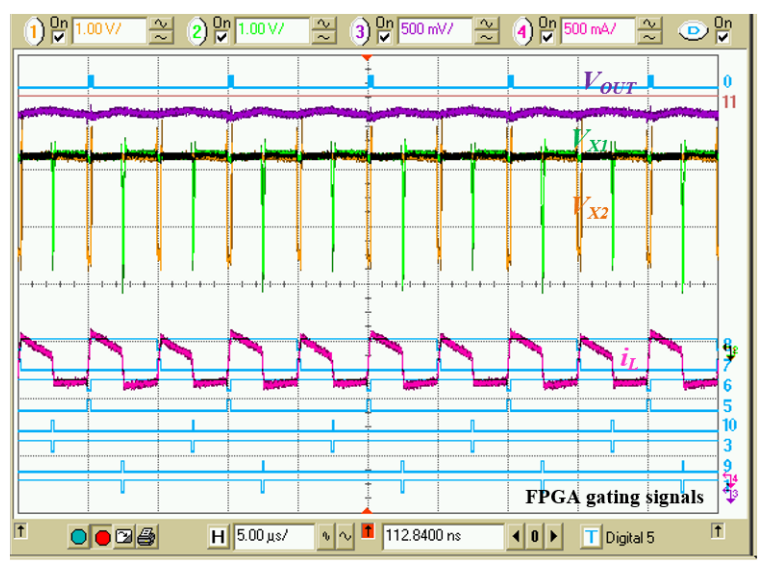

(f) RS step-up mode

Figure 4.13: Steady state operation in (a) buck (b) boost (c,d) RS step-down (e,f) RS step-up mode; For all the waveforms: Ch.1 (Orange): $2 \mathrm{~V} /$ div output switching node voltage; Ch.2 (Green): $2 \mathrm{~V} /$ div input switching node voltage; Ch.3 (Purple): $500 \mathrm{mV} /$ div output voltage; Ch.4 (Pink): $500 \mathrm{mV} /$ div inductor current; 
Fig 4.14 show mode transition between 3-level buck mode, 3-level boost mode, ML-RS step down mode and ML-RS step up mode. The zoomed in version of the corresponding transitions are shown on the right column. The results confirm fast transient and stable operation of all modes. In the zoomed in results, inductor current shape transformation can be clearly observed, which is from triangular to trapezoidal with switching frequency reduced $1 / 5$ of the previous cycle or the other way around. The worst deviation happens during the transition is under reasonable range around $3 \%$, which proves the effectiveness of the transition technique used in RS mode are still valid. No deviation on the inductor current and output voltage occurs during the transition between step-up and step-down in ML-RS. Among all the transitions, no mode toggling has been observed. 


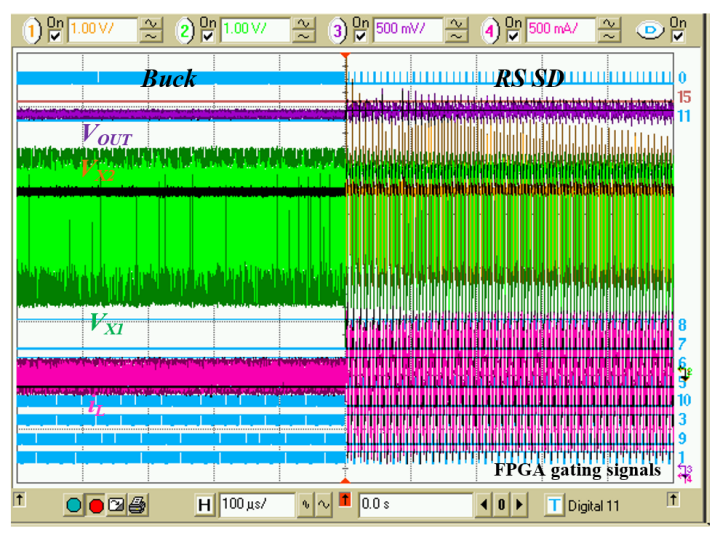

(a) Transition from buck mode to RS SD mode

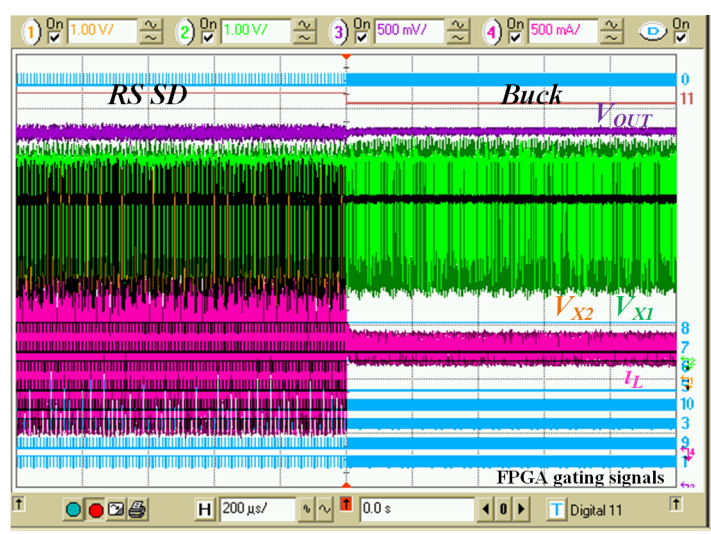

(c) Transition from RS SD mode to buck mode

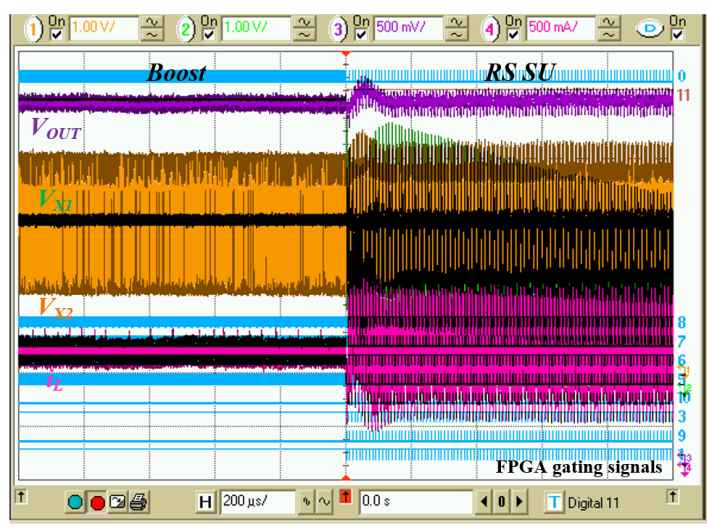

(e) Transition from boost mode to RS SU mode

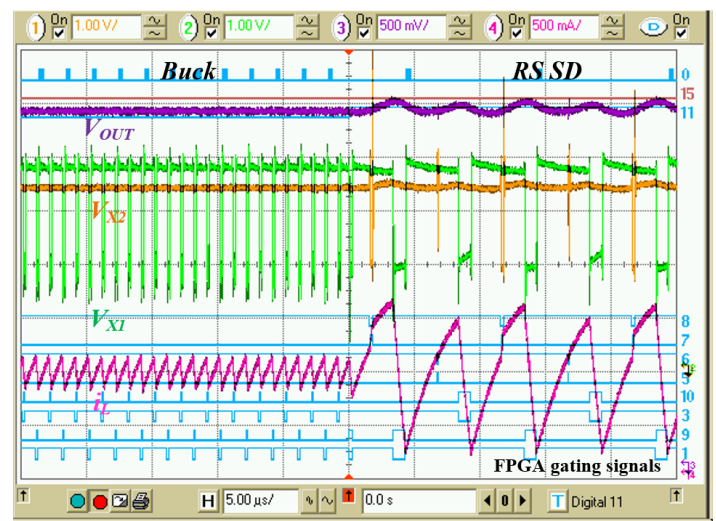

(b) Transition from buck mode to RS SD mode zoomed in

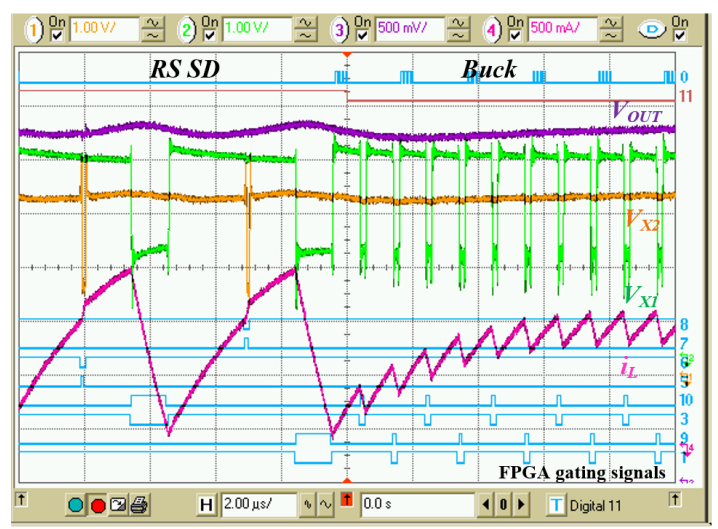

(d) Transition from RS SD mode to buck mode zoomed in

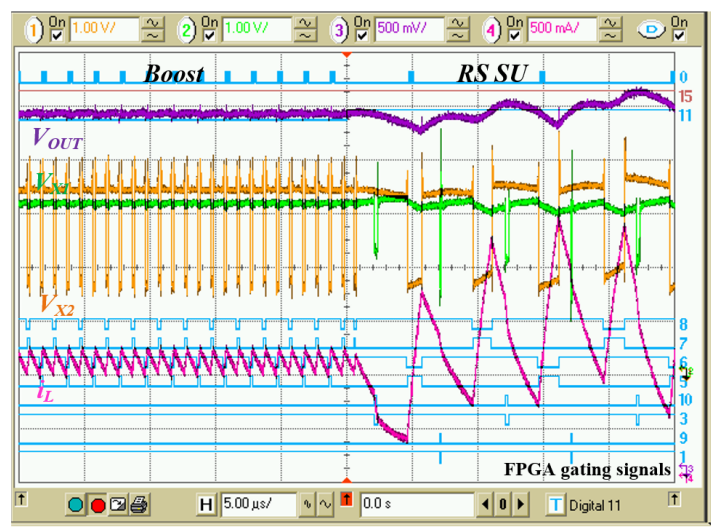

(f) Transition from boost mode to RS SU mode zoomed in 


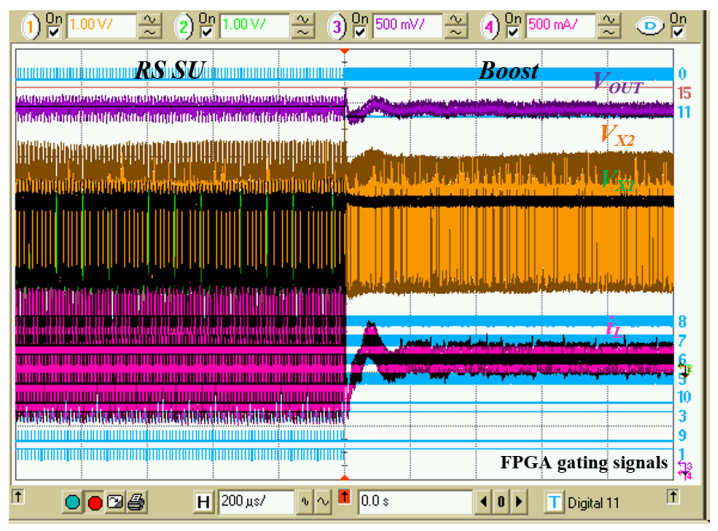

(g) Transition from RS SU mode to boost mode

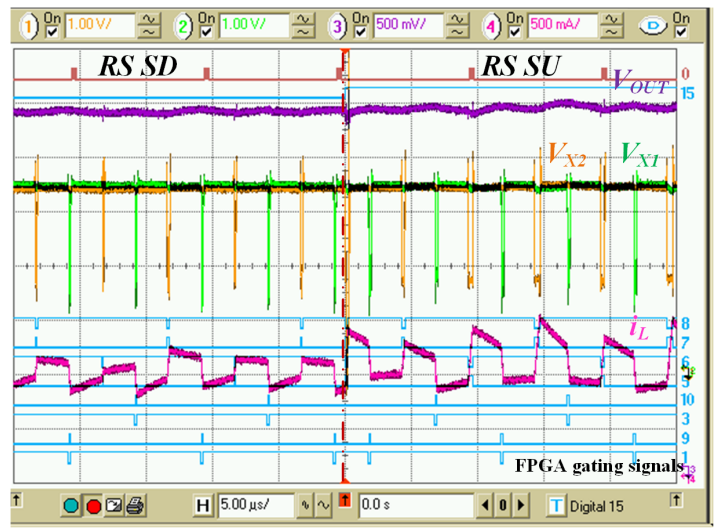

(i) Transition from RS SD mode to RS SU mode

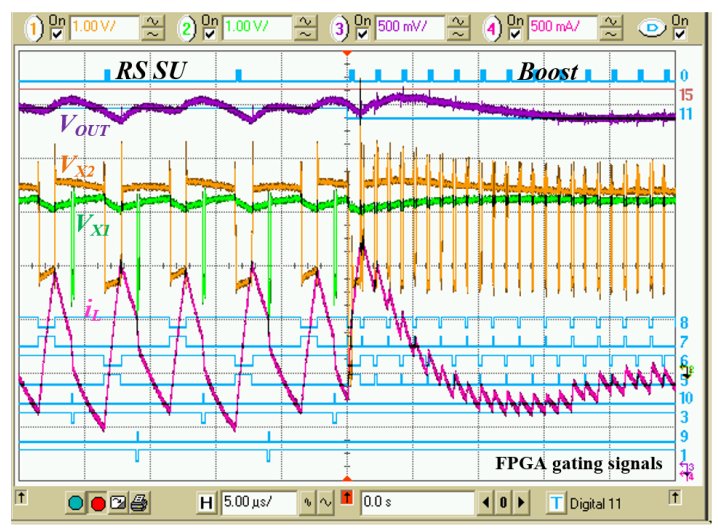

(h) Transition from RS SU mode to boost mode zoomed in

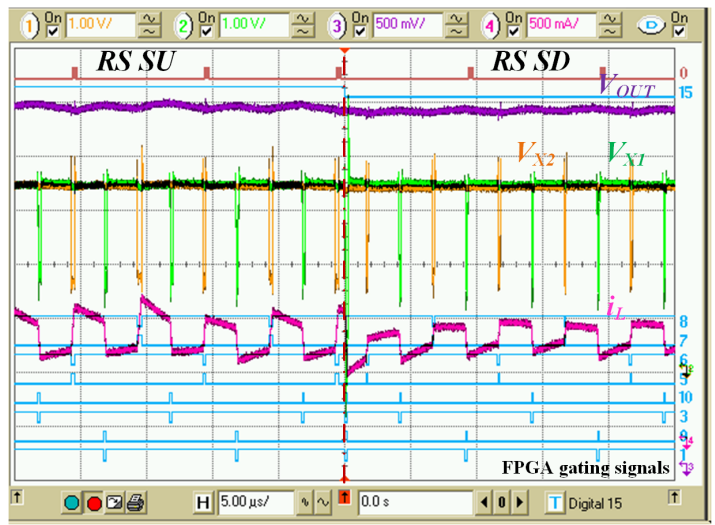

(j) Transition from RS SU mode to RS SD mode

Figure 4.14: Mode transition between (a,b,c,d) buck and RS SD (e,f,g,h) boost and RS SU $(\mathrm{i}, \mathrm{j})$ RS SD and RS SU; For all the waveforms: Ch.1 (Orange): 2V/div output switching node voltage; Ch.2 (Green): 2V/div input switching node voltage; Ch.3 (Purple): 500mV/div output voltage; Ch.4 (Pink): 500mV/div inductor current;

\subsubsection{Flying capacitor control}

The flying capacitor voltage stabilization mechanism used in conjunction with the average CPM controller is similar to the technique used in conventional voltage mode control [28], which is explored in detail in [32]. The mechanism is briefly reviewed in this section. Flying capacitors voltages are taken to compare with the reference voltage which are half of input and output voltages. A small duty ratio adjustment signal $\Delta d_{1}$ and $\Delta d_{2}$ are produced and appended to the current loop. Under the assumption that, when on time of $S W_{1}$ and $S W_{5}$ are greater than the on time of $S W_{3}$ and $S W_{7}$, the net charge 
of input and output flying capacitors are positive, i.e. flying capacitors are charging. Therefore, duty ratio adjustment signal is added to the on-time of $S W_{1,5}$. At the same time, adjustment signal will be subtracted from the on-time of $S W_{3, \gamma}$. This mechanism adjusts the charging and discharging phase of the flying capacitors by center of the duty ratio, while maintaining the constant average duty cycle. By doing so, there is no additional burden on original voltage and current loop. The duty ratio of each switch with offsetting mechanism is shown in the following equations:

$$
\begin{aligned}
& d_{S W_{1}}=d_{1}+\Delta d_{1} \\
& d_{S W_{3}}=d_{1}-\Delta d_{1} \\
& d_{S W_{5}}=d_{2}+\Delta d_{2} \\
& d_{S W_{7}}=d_{2}-\Delta d_{2}
\end{aligned}
$$

where $d_{S W 1,3,5,7}$ are duty ratio of the corresponding switches, $d_{1}$ and $d_{2}$ are average duty ratio of 3-level buck and 3-level boost, $\Delta d_{1}$ and $\Delta d_{2}$ are duty ratio adjustment signal for input and output flying capacitor stabilization.

Fig 4.15 shows the inductor current and flying capacitor current waveforms in one switching cycle when perturbations are introduced on both sides. Under the condition where $V_{\text {cflybuck }}$ is smaller than half of $V_{I N}$ and $V_{\text {cflyboost }}$ is larger than half of $V_{\text {OUT }}$. The inductor current has unsymmetrical waveform. Different charging and discharging slopes on flying capacitor further deviate the flying capacitor voltage value from the desired reference value [33]. In the case shown here, the input flying capacitor needs to be charged and output flying capacitor needs to be discharged. Fig 4.16 shows the duty ratio offsetting mechanism. When the flying capacitor voltage is perfectly balanced, error signal will lead to zero duty ratio offsetting $\Delta d$. Under the disturbance as shown in Fig 4.16, $\Delta d_{1}$ is greater than 0 , so that the charging phase for input flying capacitor is extended by $\Delta d_{1}$ while the duration of discharging phase is reduced by the same amount. The modification of two duty ratio is symmetrically around the average duty ratio. For the output flying capacitor, $\Delta d_{2}$ is smaller than 0 so that the charging phase where $S W_{5}$ is on is reduced and discharging phase is increased. Therefore, the desired flying capacitors voltage can be obtained cycle by cycle. Fig 4.17 shows the experimental results of duty ratio offsetting mechanism. On the left, by looking at both switching nodes, it can be 
noticed that both flying capacitors are not balanced and result shown on the right is the case where mechanism is enabled and desired flying capacitor voltages are obtained.

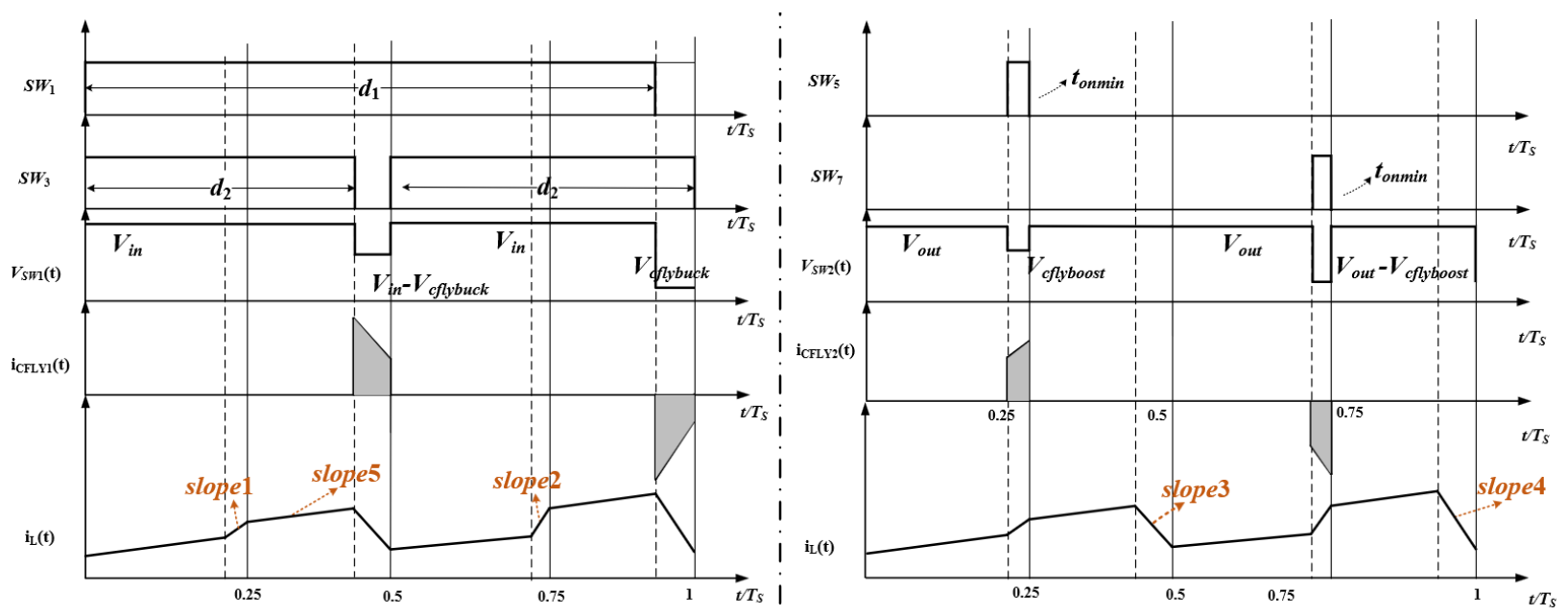

Figure 4.15: Key waveforms in ML-RS step down mode with flying capacitor voltage perturbations

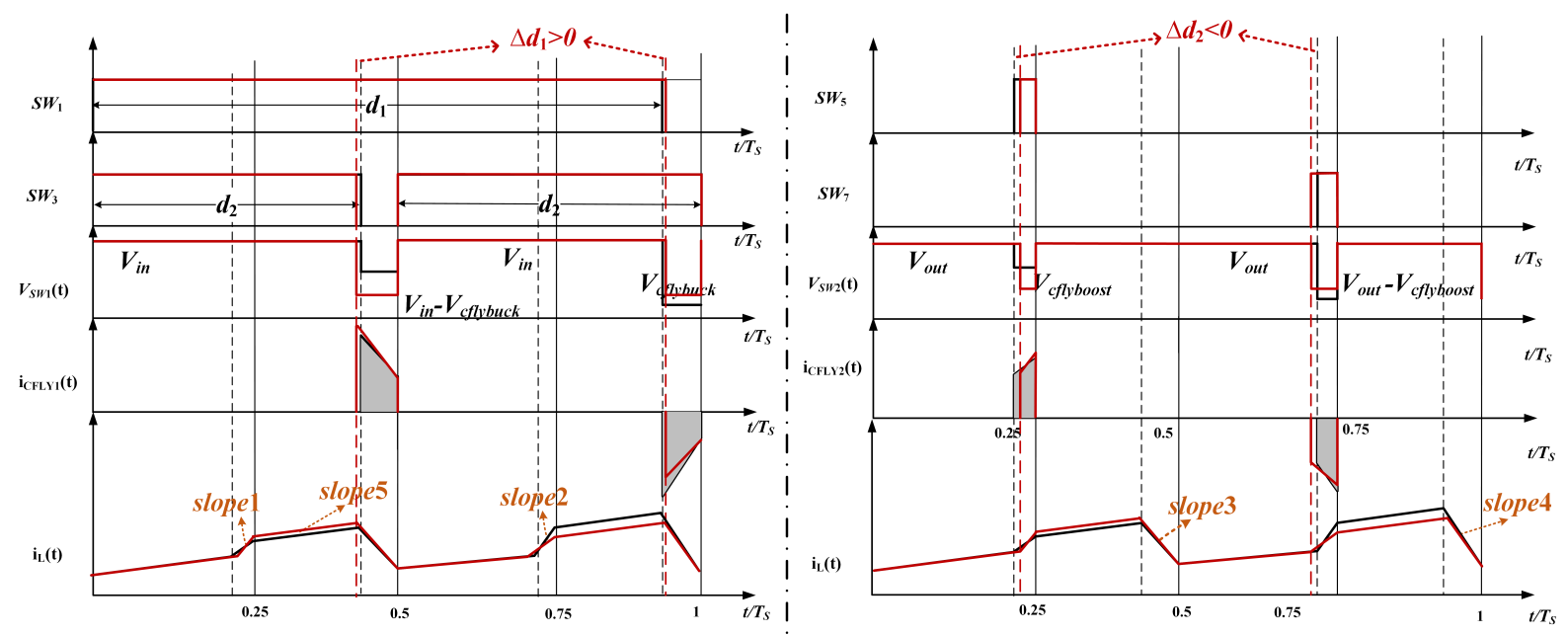

Figure 4.16: Duty ratio offsetting mechanism balance the flying capacitor voltages 


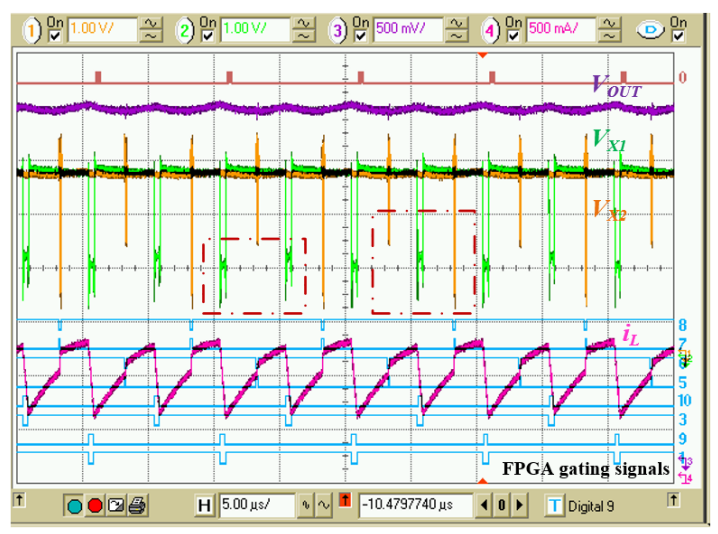

(a) ML RS step down mode without duty ratio offsetting mechanism

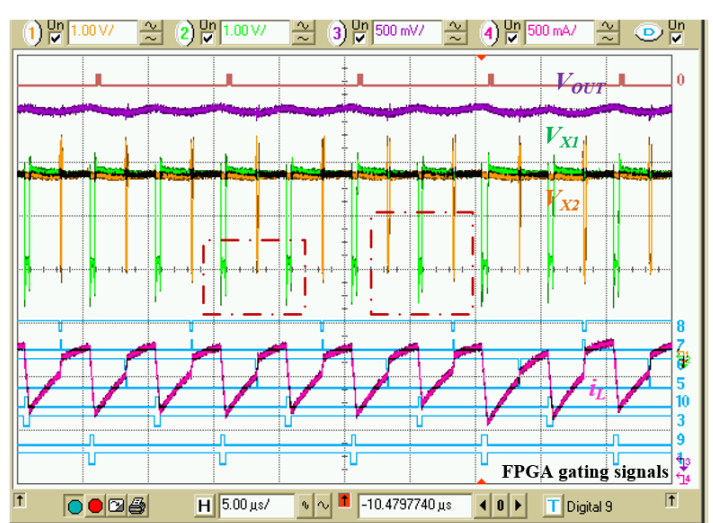

(b) ML RS step down mode with duty ratio offsetting mechanism

Figure 4.17: Duty ratio offsetting mechanism in RS mode 


\subsection{Conclusion}

The ML-NIBB converter and a complementary ML RS controller as an alternative to conventional NIBB converter are introduced in this chapter. With the utilization of two flying capacitors, voltage swing on both sides of the inductor is reduced to half, blocking voltage of power switches also reduced to half, which provides volume reduction and potentially better figure of merit(FOM). Additionally, smaller inductor has smaller winding resistance. Therefore, the topology provides higher power density through reduction of volume and improvements of power processing efficiency. Furthermore, the ML-RS controller is used as the practical solution for the transition issue in the boundary of step-down and step-up operation. The features of the RS mode are enhanced in the ML-NIBB and advantages are experimentally verified. 


\section{Chapter 5}

\section{Conclusion}

This thesis addresses the step-up and step-down transition issues in the non-inverting buck-boost converter, caused by duty ratio limitatioin in realistic converters and consequent nonlinearities in the ocnversion ratio function. The objectives are smooth transition between step-down and step-up operation, while increasing the power processing efficiency compared to state of the art solutions. To achieve these objectives, RS based dual-frequency controller is introduced. The low frequency ripple-shaping mode reshapes the inductor current from conventional triangular to trapezoidal form to reduce the inductor RMS current. On top of that, switching frequency is reduced providing switching loss reduction without affecting the inductor current ripple, i.e. RMS current. Thanks to the features of RS mode including the ability of step-up and step-down conversion ratio, low inductor current ripple and low switching frequency. Improved power processing efficiency is achieved in the transition range. To avoid large output variation at the mode transition point between buck/boost and RS mode, transition strategy is developed. Mode selection is based on the input and reference voltage and hysteretic band is implemented, to eliminate the mode toggling issues. Also, frequency scaling is used under the approximation that the duty ratio at the transition point is the same for adjacent modes with different frequencies, which keeps the duty ratio the same by scaling the frequency accordingly. And compensator reset is developed for the transition between RS step-down and RS step-up. Compensator reset technique achieves the transition in one switching cycle with almost no variation at the output voltage and inductor current. Discrete components prototype has been built to verify the operation of the introduced low-frequency ripple shaping based buck-boost controller. The controller was created using an FPGA-based evaluation board. Steady state operation of all modes that cover all conversion ratio range and smooth transition between each adjacent mode are 
achieved. Furthermore, efficiency comparison among the RS mode and other state of the art solutions that are used in commercial product verifies improvements of power processing efficiency. Also, the multi-level non-inverting buck boost converter is introduced as a further improvement and attractive alternative to the conventional NIBB converter. The comparison between both topologies is made in terms of overall volume and power processing efficiency. It has been shown that, ML-NIBB can futher reduce volume while improve power processing efficiency. RS mode is extended to ML-NIBB to achieve the smooth transition and enhance the advantages of it in multi level architectures. Efficiency measurements in all ranges show the maximum efficiency improvement is about $20 \%$ in deep boost operation in light load and around $3 \%$ in heavy load.

\subsubsection{Future work}

IC integration of ML-NIBB can be further analyzed and implemented. Other possible implementation of the mixed signal controllers can be further discussed. For example, current programmed mode hybrid hysteretic control. Current program control could potentially provide faster dynamic response during transitions. In addition, current program control provides better control of the peak current at the transition when frequency switches to much lower value in RS mode. On top of that, intrinsic flying capacitor stabilization could be potentially obtained in the ML-RS mode. According to the work presented [33], valley current mode control/peak current mode control provide intrinsic flying capacitor stabilization when duty ratio is larger/smaller than 0.5. And in the ML RS mode, the duty ratio of 3-level buck side is greater 0.5 and duty ratio of 3-level boost side is smaller than 0.5. Mode selection can be further optimized. For the target application, input voltage changes slowly and predictably. Therefore, the mode selection and mode transition technique can rely on the sensing of input voltage with hysteretic band. For other application of buck boost converter when input or output voltage change rapidly, more general transition technique will be needed. 


\section{Bibliography}

[1] S. Waffler and J. W. Kolar. A novel low-loss modulation strategy for high-power bidirectional buck-boost converters. IEEE Transactions on Power Electronics, 24 (6):1589-1599, June 2009. ISSN 0885-8993. doi: 10.1109/TPEL.2009.2015881.

[2] R. L. Lin and R. C. Wang. Non-inverting buck-boost power-factor-correction converter with wide input-voltage-range applications. In IECON 2010 - 36th Annual Conference on IEEE Industrial Electronics Society, pages 599-604, Nov 2010. doi: 10.1109/IECON.2010.5675217.

[3] E. Schaltz, P. O. Rasmussen, and A. Khaligh. Non-inverting buck-boost converter for fuel cell applications. In 2008 34th Annual Conference of IEEE Industrial Electronics, pages 855-860, Nov 2008. doi: 10.1109/IECON.2008.4758065.

[4] B. Sahu and G. A. Rincon-Mora. A low voltage, dynamic, noninverting, synchronous buck-boost converter for portable applications. IEEE Transactions on Power Electronics, 19(2):443-452, March 2004. ISSN 0885-8993. doi: 10.1109/TPEL.2003. 823196.

[5] A. Chakraborty, A. Khaligh, and A. Emadi. Combination of buck and boost modes to minimize transients in the output of a positive buck-boost converter. In IECON 2006 - 32nd Annual Conference on IEEE Industrial Electronics, pages 2372-2377, Nov 2006. doi: 10.1109/IECON.2006.347797.

[6] B. Sahu and G. A. Rincon-Mora. A high-efficiency, dual-mode, dynamic, buck-boost power supply ic for portable applications. In 18th International Conference on VLSI Design held jointly with 4th International Conference on Embedded Systems Design, pages 858-861, Jan 2005. doi: 10.1109/ICVD.2005.15.

[7] M. H. Huang and K. H. Chen. Single-inductor dual buck-boost output (sidbbo) converter with adaptive current control mode (accm) and adaptive body switch 
(abs) for compact size and long battery life in portable devices. In 2009 Symposium on VLSI Circuits, pages 164-165, June 2009.

[8] P. Rajarshi and D. Maksimovic. Analysis of pwm nonlinearity in non-inverting buckboost power converters. In 2008 IEEE Power Electronics Specialists Conference, pages 3741-3747, June 2008. doi: 10.1109/PESC.2008.4592538.

[9] P. Rajarshi and D. Maksimovic. Smooth transition and ripple reduction in 4-switch non-inverting buck-boost power converter for wcdma rf power amplifier. In 2008 IEEE International Symposium on Circuits and Systems, pages 3266-3269, May 2008. doi: 10.1109/ISCAS.2008.4542155.

[10] C. L. Wei, C. H. Chen, K. C. Wu, and I. T. Ko. Design of an average-current-mode noninverting buck-boost dc-dc converter with reduced switching and conduction losses. IEEE Transactions on Power Electronics, 27(12):4934-4943, Dec 2012. ISSN 0885-8993. doi: 10.1109/TPEL.2012.2193144.

[11] X. E. Hong, J. F. Wu, and C. L. Wei. 98.1\%-efficiency hysteretic-current-mode noninverting buck-boost dc-dc converter with smooth mode transition. IEEE Transactions on Power Electronics, 32(3):2008-2017, March 2017. ISSN 0885-8993. doi: 10.1109/TPEL.2016.2567484.

[12] P. C. Huang, W. Q. Wu, H. H. Ho, and K. H. Chen. Hybrid buck boost feedforward and reduced average inductor current techniques in fast line transient and highefficiency buck-boost converter. IEEE Transactions on Power Electronics, 25(3): 719-730, March 2010. ISSN 0885-8993. doi: 10.1109/TPEL.2009.2031803.

[13] X. Hu and P. K. T. Mok. Analysis and design of three-state controlled transition mode for a buck-boost converter with efficiency and stability enhancement. In 2013 IEEE International Symposium on Circuits and Systems (ISCAS2013), pages 697700, May 2013. doi: 10.1109/ISCAS.2013.6571942.

[14] P. C. Huang, W. Q. Wu, H. H. Ho, and K. H. Chen. High efficiency and smooth transition buck-boost converter for extending battery life in portable devices. In 2009 IEEE Energy Conversion Congress and Exposition, pages 2869-2872, Sept 2009. doi: 10.1109/ECCE.2009.5316448.

[15] A. Prodic and D. Maksimovic. Digital pwm controller and current estimator for a low-power switching converter. In COMPEL 2000. 7th Workshop on Computers 
in Power Electronics. Proceedings (Cat. No.00TH8535), pages 123-128, 2000. doi: 10.1109/CIPE.2000.904703.

[16] A. Prodic, D. Maksimovic, and R. W. Erickson. Design and implementation of a digital pwm controller for a high-frequency switching dc-dc power converter. In Industrial Electronics Society, 2001. IECON '01. The 27th Annual Conference of the IEEE, volume 2, pages 893-898 vol.2, 2001. doi: 10.1109/IECON.2001.975878.

[17] Robert W Erickson and Dragan Maksimovic. Fundamentals of power electronics. Springer Science \& Business Media, 2007.

[18] Thierry A Meynard and Henri Foch. Multilevel converters and derived topologies for high power conversion. In Industrial Electronics, Control, and Instrumentation, 1995., Proceedings of the 1995 IEEE IECON 21st International Conference on, volume 1, pages 21-26. IEEE, 1995.

[19] T. A. Meynard and H. Foch. Multi-level conversion: high voltage choppers and voltage-source inverters. In Power Electronics Specialists Conference, 1992. PESC '92 Record., 23rd Annual IEEE, pages 397-403 vol.1, Jun 1992. doi: 10.1109/PESC. 1992.254717.

[20] I2C Controlled 6A Three-Level Switch Mode Single-Cell Charger, February 2018.

[21] Robert W. Erickson David C. ones. Buck-boost converter efficiency maximization via a nonlinear digital control mapping for adaptive effective switching frequency. In IEEE Journal of Emerging and Selected Topics in Power Electronics, volume 1, pages 153 - 165. IEEE, 2013.

[22] Y. J. Lee, A. Khaligh, and A. Emadi. A compensation technique for smooth transitions in non-inverting buck-boost converter. In 2009 Twenty-Fourth Annual IEEE Applied Power Electronics Conference and Exposition, pages 608-614, Feb 2009. doi: 10.1109/APEC.2009.4802721.

[23] C. W. Chang and C. L. Wei. Single-inductor four-switch non-inverting buck-boost dc-dc converter. In Proceedings of 2011 International Symposium on VLSI Design, Automation and Test, pages 1-4, April 2011. doi: 10.1109/VDAT.2011.5783629.

[24] A. Cid-Pastor A. E. Aroudi R. Giral C. Restrepo, J. Calvente. A noninverting buckboost dcde switching converter with high efficiency and wide bandwidth. In IEEE Transactions on Power Electronics, volume 26, pages 2490 - 2503. IEEE, 2011. 
[25] Texas Instruments. TPS63070 2-V to 16-V Buck-Boost Converter With 3.6-A Switch Current, August 2016.

[26] Johann W. Kolar Stefan Waffler. A novel low-loss modulation strategy for highpower bidirectional buck + boost converters. In IEEE Transactions on Power Electronics, volume 24, pages 1589 - 1599. IEEE, 2009.

[27] Maxim application note AN-1205. W-CDMAPower Supply Dramatically Improves Transmit Efficiency, October 2001.

[28] Nenad Vukadinovic. Low-Power Multi-Level Flying Capacitor Converters - Modelling and Control. PhD thesis, University of Toronto, 2017.

[29] WE-MAPI SMD Shielded Power Inductor. Wrth Elektronik, Nov 2015. application note.

[30] IHLP Commercial Inductors, Low DCR Series. VISHAY, Oct 2017. application note.

[31] B. J. Baliga. Fundamentals of Power Semiconductor Devices. Springer Publishing Company, Incorporated, 2008.

[32] L. Lu, Y. Zhang, S. M. Ahsanuzzaman, A. Prodic, G. Calabrese, G. Frattini, and M. Granato. Digital average current programmed mode control for multi-level flying capacitor converters. In Workshop on Control and Modeling for Power Electronics(COMPEL), June 2018(In Press).

[33] L. Lu, S. M. Ahsanuzzaman, A. Prodic, G. Calabrese, G. Frattini, and M. Granato. Peak offsetting based cpm controller for multi-level flying capacitor converters. In 2018 IEEE Applied Power Electronics Conference and Exposition (APEC), pages 3102-3107, March 2018. doi: 10.1109/APEC.2018.8341543. 